$$
\begin{gathered}
\text { Universidade de São Paulo } \\
\text { Instituto de Física de São Carlos }
\end{gathered}
$$

Raul Ribeiro Prado

Estudo da composição de raios cósmicos de altas energias através da análise de dados medidos pelo Observatório Pierre Auger 



\section{Estudo da composição de raios cósmicos de altas energias através da análise de dados medidos pelo Observatório Pierre Auger}

Dissertação apresentada ao Programa de PósGraduação em Física do Instituto de Física de São Carlos da Universidade de São Paulo, para obtenção do título de Mestre em Ciências.

Área de Concentração: Física Básica

Orientador: Prof. Dr. Luiz Vitor de Souza Filho

Versão Corrigida

(versão original disponível na Unidade que aloja o Programa)

São Carlos 
AUTORIZO A REPRODUÇÃO E DIVULGAÇÃO TOTAL OU PARCIAL DESTE TRABALHO, POR QUALQUER MEIO CONVENCIONAL OU ELETRÔNICO PARA FINS DE ESTUDO E PESQUISA, DESDE QUE CITADA A FONTE.

Ficha catalográfica elaborada pelo Serviço de Biblioteca e Informação do IFSC, com os dados fornecidos pelo(a) autor(a)

Prado, Raul Ribeiro

Estudo da composição de raios cósmicos de altas energias através da análise de dados medidos pelo

Observatório Pierre Auger / Raul Ribeiro Prado;

orientador Luiz Vitor de Souza Filho - versão corrigida -- São Carlos, 2014.

$126 \mathrm{p}$.

Dissertação (Mestrado - Programa de Pós-Graduação em Física Básica) -- Instituto de Física de São Carlos, Universidade de São Paulo, 2014.

1. Raios cósmicos de altas energias. 2. Composição de raios cósmicos. 3. Análise de dados. 4.

Observatório Pierre Auger. I. de Souza Filho, Luiz Vitor, orient. II. Título. 


\section{AGRADECIMENTOS}

Em primeiro lugar agradeço ao meu pai Sergio, à minha mãe Leila e ao meu irmão Henrique. Esse mestrado e todas outras realizações em minha vida só foram possíveis graças à vocês. Agradecimentos especiais também à minha vó Vita, ao meu tio Val, ao meu primo André, à Laís, à Tata, à minha amiga Laura e a toda minha família por estarem sempre próximos e me apoiando.

Agradeço ao Prof. Luiz Vitor pela orientação, científica e pessoal, ao longo dos últimos 5 anos. Agradeço aos parceiros de grupo e de sala, Jessica Dipold, Carlo Todero, Thiago Mosqueiro, Hendrik Marques, Milena Carvalho, Jéssica Marcomini, Rita de Cassia e Rodrigo Lang pelas ajudas cotidianas e por tornarem mais agradável meu ambiente de trabalho.

Agradeço à todos meus amigos e colegas que me acompanharam durante esses anos de mestrado e graduação. Agradeço aqueles que compartilharam comigo os intervalos pro café, os bandejões, as sessões de cinema e ainda aqueles que conheci na comissão organizadora da SIFSC e que acabaram se tornando bons amigos. Agradeço especialmente à Jessica Dipold, Evandro Ribeiro, Kríssia de Zawadzki, Joseana Soares, Gustavo Salinas, Vinícius Auríchio, Gilson Campani e Luis Felipe Mendes pelo intenso apoio e companhia.

Agradeço ao José Bellido pelo apoio essencial durante o período que passei em Adelaide. Agradeço a toda colaboração do Observatório Pierre Auger, em especial aos brasileiros, pelas contribuições fundamentais para a realização desse trabalho.

Agradeço a todos meus professores do IFSC por todos os ensinamentos decisivos pra minha formação como cientista. Agradeço também a toda equipe de servidores do IFSC (o pessoal da secretaria, da biblioteca, da gráfica e todos os outros) por fornecerem a estrutura necessária para a realização desse mestrado.

Finalmente, agradeço à FAPESP pelo apoio financeiro. 

"There is a theory which states that if ever anyone discovers exactly what the Universe is for and why it is here, it will instantly disappear and be replaced by something even more bizarre and inexplicable.

There is another theory which states that this has already happened."

Douglas Adams (The Restaurant at the End of the Universe, 1980) 



\section{RESUMO}

PRADO, R. R. Estudo da composição de raios cósmicos de altas energias através da análise de dados medidos pelo Observatório Pierre Auger. 2014. 126 p. Dissertação (Mestrado em Ciências) - Instituto de Física de São Carlos, Universidade de São Paulo, São Carlos, 2014.

O conhecimento sobre a composição de raios cósmicos de altas energias é fundamental na abordagem da maior parte das grandes questões referentes à astrofísica de altas energias. Entretanto, do ponto de vista experimental, determinar o tipo de partícula medida nesse regime de energia ainda é um enorme desafio e essa tarefa tem recebido especial atenção por parte das colaborações responsáveis pelos experimentos em atividade. A principal dificuldade está no fato das medidas serem realizadas indiretamente através das cascatas de partículas formadas a partir da interação do raio cósmico inicial com átomos da atmosfera, os chamados chuveiros atmosféricos. Entre os principais experimentos em funcionamento, o Observatório Pierre Auger se destaca por ter a maior área de detecção $\left(3000 \mathrm{~km}^{2}\right)$ e por utilizar pioneiramente um sistema híbrido de detecção, com detectores de superfície e de fluorescência funcionando simultaneamente. Os telescópios de fluorescência medem o número de partículas do chuveiro em função da profundidade, o que chamamos de perfil longitudinal. Alguns parâmetros extraídos desse perfil são sensíveis à composição das partículas primárias. No presente trabalho, aplicamos métodos estatísticos novos aos dados extraídos dos perfis longitudinais de chuveiros medidos pelo Auger com o objetivo de inferir informações sobre a massa média, ou seja, a composição dos raios cósmicos. A primeira análise apresentada é baseada no parâmetro $X_{\max }$. A evolução do valor médio de $X_{\max }$ com a energia contém informações sobre a composição inicial. Com o objetivo de eliminar vieses experimentais, corrigindo os efeitos dos detectores, aplicamos métodos de deconvolução às distribuições de $X_{\max }$. A segunda análise é do tipo multiparamétrica e aplica redes neurais do tipo Multilayer Perceptrons a outros parâmetros extraídos dos perfis longitudinais. A partir desse procedimento é possível obter informações sobre a composição média das partículas e também reconstruir a energia dos eventos.

Palavras-chave: Raios cósmicos de altas energias. Composição de raios cósmicos. Análise de dados. Observatório Pierre Auger. Métodos de deconvolução. Redes neurais. 



\section{ABSTRACT}

PRADO, R. R. High energy cosmic rays composition study by the analysis of the Pierre Auger Observatory measured data. 2014. 126 p. Dissertação (Mestrado em Ciências) - Instituto de Física de São Carlos, Universidade de São Paulo, São Carlos, 2014.

The knowledge about high energy cosmic rays composition is fundamental to approach most of the big questions regarding high energy astrophysics. However, from the experimental point of view, to determine the kind of the measured particle in this energy range is still a huge challenge and this task has received special attention from the collaborations responsible for running the experiments in activity. The main difficulty is on the fact that the measurements are made indirectly by the secondary particles cascades formed by the interaction of primary particles with atmosphere atoms, which are called air showers. Among the main experiments in operation, Pierre Auger Observatory has the larger collecting area $\left(3000 \mathrm{~km}^{2}\right)$ and uses a pioneer hybrid detection system, with surface detectors and fluorescence telescopes working simultaneously. The fluorescence telescopes measure the number of particles in the shower as a function of atmospheric depth, which we call longitudinal profiles. Some parameters extracted from these profiles are sensitive to primary composition. In this study, we applied new statistical methods to the data from longitudinal profiles measured by the Pierre Auger Observatory aiming to infer information about the mean mass, in other words, the composition of cosmic rays. The first analysis shown (chapter 4 ) is based on the known parameter called $X_{\max }$. The evolution of $X_{\max }$ mean value with energy contains information about primary composition. Unfolding methods have been applied to the $X_{\max }$ distribution in order to minimize experimental bias and to correct detector effects. The second analysis shown is of the multi-parametric type and applies neural networks of the Multilayer Perceptrons class to longitudinal profiles parameters. From this procedure, it is possible to obtain information about average composition and to reconstruct the energy of events.

Keywords: High energy cosmic rays. Cosmic rays composition. Data analysis. Pierre Auger Observatory. Unfolding methods. Neural networks. 



\section{LISTA DE FIGURAS}

Figura 2.1 - Diagrama de Hillas mostrando possíveis fontes astrofísicas capazes de acelerar raios cósmicos. Fonte: Imagem extraída da referência (15). . . 28

Figura 2.2 - Espectro de energia dos raios cósmicos. Fonte: Imagem disponível em http://www.perdaix.de/cosmic_radiation.

Figura 2.3 - Espectro de energia dos raios cósmicos acima de $10^{14.0} \mathrm{eV}$. O fluxo está multiplicado por $E^{2.6}$ para facilitar a visualização das estruturas. Fonte: Imagem extraída da referência (24) . . . . . . . . . . . . . . . 31

Figura 2.4 - Esquema do desenvolvimento de um chuveiro atmosférico. Fonte: Imagem disponível em http://commons.wikimedia.org/wiki/File:AirShower.svg. 32

Figura 2.5 - Seção de choque próton-ar medida por vários experimentos e obtidas através de modelos teóricos. Fonte: Imagem extraída da referência (26). 33

Figura 2.6 - Medidas de $\left\langle X_{\max }\right\rangle$ em função da energia para vários experimentos entre (a) $10^{15.0}$ e $10^{20.0} \mathrm{eV}$ e (b) $10^{17.8}$ e $10^{20.0} \mathrm{eV}$. Os dados foram extraídos da referência (27). Fonte: Imagem elaborada pelo autor. . . . . . . . . 36

Figura 2.7 - Gráficos do $R M S$ do $X_{\max }$ (esquerda) e da variância da gaussiana ajustada às distribuições de $X_{\max }$ (direita). Fonte: Imagem extraída da referência (27).

Figura 2.8 - Espectro de energia medido pelo KASCADE-Grande (40). Os dados podem ser encontrados na referência (42). Fonte: Imagem extraída da referência (43).

Figura 3.1 - Configuração do Observatório Pierre Auger em março de 2009. Os pontos cinza representam as posições dos detectores de superfície e a linhas indicam o campo de visão de cada um dos telescópios de fluorescência. Fonte: Imagem extraída da referência (56).

Figura 3.2 - Foto de uma das estações do detector de superfície do Observatório Pierre Auger. 
Figura 3.3 - (a) Desenho de um dos telescópios de fluorescência. Fonte: Imagem extraída da referência (56). (b) Fotografia de um dos telescópios de fluorescência do Observatório Pierre Auger. Fonte: Imagem disponível em http://www.auger.org/observatory/gallery2005.html. . . . . . . . . 42

Figura 3.4 - Representação esquemática da geometria dos chuveiros medidos pelos telescópios de fluorescência, mostrando os parâmetros geométricos utilizados na reconstrução. Fonte: Imagem extraída da referência (65).

Figura 3.5 - (a) Gráfico mostrando os pixeis de um dos telescópios ao medir um chuveiro atmosférico. A escala de cores representa o tempo da medida. (b) Dados de $t \times \chi$ para um evento medido pelo telescópio ajustado pela função mostrada na equação 3.5. O ponto preto representa o hottest station usada na reconstrução híbrida. Fonte: Imagens elaboradas pelo

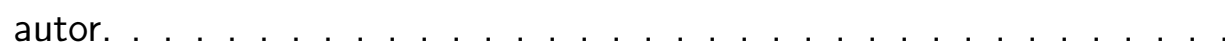

Figura 3.6 - Função de distribuição lateral de um evento medido pelo Observatório Pierre Auger. Os pontos pretos mostram os sinais medidos por cada estação e a curva azul é uma função dada pela equação 3.8 ajustada aos dados. Fonte: Imagem extraída da referência (71) . . . . . . . . . . .

Figura 3.7 - Curva de atenuação utilizada para obter o parâmetro $S_{38}$. Os pontos mostram a média do sinal medido em um intervalo de ângulo zenital. Em cada intervalo, estão incluídos todos os eventos medidos pelo Observatório Pierre Auger que sobreviveram aos cortes de qualidade desta análise. Fonte: Imagem extraída da referência (73). . . . . . . . . . . . 48

Figura 3.8 - Exemplo de um perfil longitudinal medido pelos telescópios de fluorescência. Os pontos pretos são os sinais medidos pelos telescópios em cada intervalo de profundidade atmosférica. O ponto vermelho é o valor de $X_{\max }$ ajustado. A curva vermelha é a função Gaisser-Hillas ajustada aos dados. Fonte: Imagem elaborada pelo autor. . . . . . . . . . . . .

Figura 3.9 - Relação entre $S_{38}$ e $E_{F D}$ para um subconjunto de eventos medidos pelo Observatório Pierre Auger. A linha cinza é a função utilizada para obter a energia da partícula primária a partir do parâmetro $S_{38}$, para os eventos medidos apenas pelos detectores de superfície. Fonte: Imagem extraída da referência (77) . . . . . . . . . . . . . . . . . . . . . . . 50 
Figura 4.1 - Distribuição de $X_{\max }$ de chuveiros iniciados por prótons com energia de $10^{18.6} \mathrm{eV}$, gerados pelo CONEX. Fonte: Imagem gerada pelo autor. . 60

Figura 4.2 - Correlação entre $X_{0}$ e $\lambda$ para chuveiro simulados com energia igual a $10^{18.6} \mathrm{eV}$. Fonte: Imagem elaborada pelo autor.

Figura 4.3 - Teste de consistência para $\left\langle X_{\max }\right\rangle$. Estão mostrados os valores de $\left\langle X_{\max }\right\rangle$ para as distribuição reais e deconvoluídas pelos dois métodos e para dois tipos de primários diferentes, prótons e núcleos de ferro. Fonte: Imagem elaborada pelo autor.

Figura 4.4 - Teste de consistência para $R M S_{X_{\max }}$. Estão mostrados os valores de $R M S_{X_{\max }}$ para as distribuição reais e deconvoluídas pelos dois métodos e para dois tipos de primários diferentes, prótons e núcleos de ferro. Fonte: Imagem elaborada pelo autor.

Figura 4.5 - Diferença entre os valores reais e deconvoluídos em função da energia para (a) $\left\langle X_{\max }\right\rangle$ e (b) $R M S_{X_{\max }}$. Fonte: Imagem elaborada pelo autor. 66

Figura 4.6 - Número de eventos selecionados para essa análise (somente cortes de qualidade) comparado com o número de eventos selecionados para a análise padrão (cortes de qualidade e fidelidade). Fonte: Imagem elaborada pelo autor.

Figura 4.7 - Incertezas estatísticas em (a) $\left\langle X_{\max }\right\rangle$ e (b) $R M S_{X_{\max }}$ para o método SVD. A incerteza padrão está indicado como $\sigma^{\prime}$ e a incerteza calculada pelo toy Monte Carlo está indicado como $\sigma^{M C}$. Fonte: Imagem elaborada pelo autor.

Figura 4.8 - Incertezas estatísticas em (a) $\left\langle X_{\max }\right\rangle$ e (b) $R M S_{X_{\max }}$ para o método bayesiano. A incerteza padrão está indicado como $\sigma^{\prime}$ e a incerteza calculada pelo toy Monte Carlo está indicado como $\sigma^{M C}$. Fonte: Imagem elaborada pelo autor

Figura 4.9 - Incertezas sistemáticas em (a) $\left\langle X_{\max }\right\rangle$ e (b) $R M S_{X_{\max }}$ em função da energia para cada fonte separadamente. Fonte: Imagem elaborada pelo autor.

Figura $4.10-\chi_{n, n-1}^{2}$ em função do número de iterações $n$ para todos os bins de energia. A linha vermelha indica $\chi_{n, n-1}^{2}=0.15$. Fonte: Imagem elaborada pelo autor. 
Figura $4.11-\left\langle X_{\max }\right\rangle$ em função da energia para os dois métodos de deconvolução. Fonte: Imagem elaborada pelo autor

Figura 4.12 - $R M S_{X_{\max }}$ em função da energia para os dois métodos de deconvolução. Fonte: Imagem elaborada pelo autor. . . . . . . . . . . . . . . . 74

Figura $4.13-\left\langle X_{\max }\right\rangle$ em função da energia. As linhas mostram predições calculadas através de diferentes modelos de interação hadrônica para próton e núcleos de ferro. Fonte: Imagem elaborada pelo autor. . . . . . . . . . 75

Figura $4.14-R M S_{X_{\max }}$ em função da energia. As linhas mostram predições calculadas através de diferentes modelos de interação hadrônica para próton e núcleos de ferro. Fonte: Imagem elaborada pelo autor. . . . . . . . . 76

Figura 4.15 -(a) $\left\langle X_{\max }\right\rangle$ para o método SVD, (b) $R M S_{X_{\max }}$ para o método SVD, (c) $\left\langle X_{\max }\right\rangle$ para o método bayesiano e (d) $R M S_{X_{\max }}$ para o método bayesiano. As bandas preenchidas mostram as incertezas sistemáticas calculados na seção 4.4.3. Fonte: Imagens elaboradas pelo autor. . . . 77

Figura 4.16 - $\operatorname{Ln}(A)$ em função da energia calculado através dos valores de $\left\langle X_{\max }\right\rangle$ deconvoluídos pelos métodos SVD e bayesiano. Fonte: Imagem elaborada pelo autor.

Figura $4.17-\left\langle X_{\max }\right\rangle$ em função da energia $\left(>10^{15.0}\right)$ para os resultados diferentes experimentos comparados com os resultados obtidos nesse trabalho. Os dados de outros experimentos foram extraídos da referência (27). Fonte: Imagem elaborada pelo autor.

Figura $4.18-\left\langle X_{\max }\right\rangle$ em função da energia $\left(>10^{18.0}\right)$ para os resultados diferentes experimentos comparados com os resultados obtidos nesse trabalho. Os dados de outros experimentos foram extraídos da referência (27). Fonte: Imagem elaborada pelo autor. . . . . . . . . . . . . . . . . . 79

Figura 4.19 -Distribuições de (a) $X_{0}$, (b) $\lambda$ e (c) $\log \left(N_{\max }\right)$. O bin de energia é definido por $18.45<\log (E / e V)<18.5$ e o bin de $X_{\max }$ é definido por $700<X_{\max } /\left(\mathrm{g} / \mathrm{cm}^{2}\right)<725$. As curvas em vermelho mostram as funções gaussianas ajustadas às distribuições. Fonte: Imagens elaboradas pelo autor. 
Figura 4.20 -Parametrização de (a) $\mu_{X_{0}}$, (b) $\sigma_{X_{0}}$, (c) $\mu_{\lambda}$, (d) $\sigma_{\lambda}$, (e) $\mu_{\log \left(N_{\max }\right)}$ e (f) $\sigma_{\log \left(N_{\max }\right)}$ em função de $X_{\max }$. O bin de energia é definido por $18.4<\log (E / e V)<18.5$. As curvas em vermelho são polinômios de segundo grau ajustados aos gráficos. Fonte: Imagens elaboradas pelo autor. . . . . . . . . . . . . . . . . 86

Figura 4.21 -Parametrização final de (a) $\mu_{X_{0}}$, (b) $\sigma_{X_{0}}$, (c) $\mu_{\lambda}$, (d) $\sigma_{\lambda}$, (e) $\mu_{\log \left(N_{\max }\right)}$ e (f) $\sigma_{\log \left(N_{\max }\right)}$ em função da energia (eixo $x$ ) $X_{\max }$ (eixo $y$ ). Os códigos das cores representam os parâmetros $\sigma^{\prime} s$ e $\mu^{\prime} s$. As linhas pretas definem os limites de utilização da parametrização. Fonte: Imagens elaboradas pelo autor.

Figura 4.22 -Matriz de resposta do detector para o intervalo de energia definido por $18.4<\log (E / e V)<18.5$. Fonte: Imagem elaborada pelo autor. . . . 88

Figura 4.23 -Matrizes resposta do detector para os nove primeiros intervalos de energia. Fonte: Imagem elaborada pelo autor.

Figura 4.24 -Matrizes resposta do detector para os nove últimos intervalos de energia. Fonte: Imagem elaborada pelo autor.

Figura 4.25 -Distribuições de $X_{\max }$ deconvoluídas para os dois métodos de deconvolução, SVD e bayesiano. Cada histograma representa um bin de energia. Fonte: Imagem elaborada pelo autor.

Figura 5.1 - Funções de ativação dos tipos (a) linear, (b) threshold, (b) threshold linear e (d) sigmoid. Fonte: Imagem extraída da referência (6) . . . . . 93

Figura 5.2 - Exemplo de um perfil longitudinal medido pelo Observatório Pierre Auger e ajustado pela função gaussiana na idade do chuveiro. $N$ é o número de partículas e $s$ é a idade do chuveiro. Os pontos pretos são os valores medidos e a curva vermelha é a função ajustada. Fonte: Imagem elaborada pelo autor.

Figura 5.3 - Gráfico de $\log \left(N_{\max }\right) \times \log (E)$ mostrando a forte correlação do parâmetro $\log \left(N_{\max }\right)$ com a energia primária do chuveiro. Os dados foram extraídos de um conjunto de chuveiros simulados pelo software CONEX (30, 31) e reconstruídos pelo Offline (95). O modelo de interação hadrônica utilizado foi o QGSJetlI-04 (99, 100). Fonte: Imagem elaborada pelo autor. . . . . . . . . . . . . . . . . . . . 995 
Figura 5.4 - Distribuições das diferenças entre os parâmetros reconstruídos e simulados, para (a) $X_{\max }$, (b) $\sigma$ e (c) $\log \left(N_{\max }\right)$. O intervalo de energia dos eventos selecionados para esse exemplo é $19.0<\log (E / \mathrm{eV})<19.2$. As distribuições estão normalizadas para facilitar a comparação entre diferentes primários. Fonte: Imagem elaborada pelo autor. . . . . . . . 97

Figura 5.5 - Resolução dos parâmetros (a) $X_{\max }$, (b) $\sigma$ e (c) $\log \left(N_{\max }\right)$ em função da energia obtida através de chuveiros simulados. Fonte: Imagem elaborada pelo autor. . . . . . . . . . . . . . . . . . . . . . . . . . . . 98

Figura 5.6 - Número de eventos simulados para cada tipo de primário por intervalo de energia. Fonte: Imagem elaborada pelo autor. . . . . . . . . . . . . 99

Figura 5.7 - Número de eventos selecionados para essa análise para cada bin de energia. Fonte: Imagem elaborada pelo autor. . . . . . . . . . . . . . . 100

Figura 5.8 - Gráficos de (a) $X_{\max } \times s_{\text {inf }}$ e (b) $X_{\max } \times s_{\text {sup }}$. As linhas pretas mostram os limites $s_{\text {inf }}=0.8$ e $s_{\text {sup }}=1.2$ usados para separar os eventos enviesados e os não enviesados. Fonte: Imagem elaborada pelo autor. . 101

Figura 5.9 - Gráficos de (a) $\sigma \times s_{\text {inf }}$ e (b) $\sigma \times s_{\text {sup. }}$. As linhas pretas mostram os limites $s_{\text {inf }}=0.8$ e $s_{\text {sup }}=1.2$ usados para separar os eventos enviesados e os não enviesados. Fonte: Imagem elaborada pelo autor. . 102

Figura 5.10 -Estrutura da rede neural usada por esse trabalho. Fonte: Imagem elaborada pelo autor. . . . . . . . . . . . . . . . . . . . 103

Figura 5.11 -Fator de mérito (FM) em função da energia para os parâmetros $X_{\max }$, $\sigma, \log \left(N_{\max }\right)$ e $M L P$. O FM foi calculado usando as distribuições dos parâmetros de chuveiros simulados iniciados por prótons e núcleos de ferro. Fonte: Imagem elaborada pelo autor. . . . . . . . . . . . . 105

Figura 5.12 -Fator de mérito calculado para o parâmetro $M L P$ considerando os três parâmetros de entrada $\left(X_{\max }, \sigma, \log \left(N_{\max }\right)\right)$ e pares desses. Fonte: Imagem elaborada pelo autor. . . . . . . . . . . . . . . . 106

Figura 5.13 -Evolução do $X_{\max }$ com a energia para simulações de núcleos de hidrogênio e ferro e para os dados medidos pelo Observatório Pierre Auger. Fonte: Imagem elaborada pelo autor. . . . . . . . . . . . . . . . . 107 
Figura 5.14 -Evolução do $\sigma$ com a energia para simulações de núcleos de hidrogênio e ferro e para os dados medidos pelo Observatório Pierre Auger. Fonte: Imagem elaborada pelo autor.

Figura 5.15 -Evolução do parâmetro $M L P$ com a energia para simulações de núcleos de hidrogênio e ferro e para os dados medidos pelo Observatório Pierre Auger. Fonte: Imagem elaborada pelo autor. . . . . . . . . . . . . . . 108

Figura 5.16 -Evolução do parâmetro $M L P$ com a energia para simulações de núcleos de hidrogênio e hélio e para os dados medidos pelo Observatório Pierre Auger. Fonte: Imagem elaborada pelo autor. . . . . . . . . . . . . . . 108

Figura 5.17 -Evolução do parâmetro $\alpha$ com a energia. Fonte: Imagem elaborada pelo autor.

Figura 5.18 -Resolução energética para a análise padrão e a análise desse trabalho obtida através de chuveiros simulados. Fonte: Imagem elaborada pelo autor. . . . . . . . . . . . . . . . . . . . . 110

Figura 5.19 -Resolução energética para a análise desse trabalho usando $X_{\max }, \sigma$ e $\log \left(N_{\max }\right)$ e usando pares desses parâmetros. Fonte: Imagem elaborada pelo autor.

Figura 5.20 -Diferença entre a energia reconstruída pelo método de redes neurais, apresentada nesse trabalho, e a energia reconstruída pelo método padrão para os dados medidos pelo Observatório Pierre Auger. Fonte: Imagem elaborada pelo autor.

Figura 6.1 - Resumo dos resultados apresentados nessa dissertação. O primeiro gráfico mostra o $\left\langle X_{\max }\right\rangle$ em função da energia para os métodos de deconvolução. O segundo mostra o $R M S_{X_{\max }}$ em função da energia para os métodos de deconvolução. O último mostra o parâmetro $\langle M L P\rangle$ obtido através da análise por redes neurais em função da energia. Fonte: Imagem elaborada pelo autor. 



\section{LISTA DE TABELAS}

Tabela 4.1 - Parametrização de $\mu_{\log 10\left(N_{\max }\right)}$ e $\sigma_{\log 10\left(N_{\max }\right)}$ em função de $X_{\max }$. Os parâmetros mostrados são referentes as funções $\mu_{\log 10\left(N_{\max }\right)}=a_{0}+$ $a_{1} \cdot X_{\max }+a_{2} \cdot X_{\max }^{2}$ e $\sigma_{\log 10\left(N_{\max }\right)}=b_{0}+b_{1} \cdot X_{\max }+b_{2} \cdot X_{\max }^{2} . \quad .80$

Tabela 4.2 - Parametrização de $\mu_{\lambda}$ e $\sigma_{\lambda}$ em função de $X_{\max }$. Os parâmetros mostrados são referentes as funções $\mu_{\lambda}=a_{0}+a_{1} \cdot X_{\max }+a_{2} \cdot X_{\text {max }}^{2} \mathrm{e}$ $\sigma_{\lambda}=b_{0}+b_{1} \cdot X_{\max }+b_{2} \cdot X_{\max }^{2} \ldots \ldots \ldots \ldots$. . . . . . . 81

Tabela 4.3 - Parametrização de $\mu_{X_{0}}$ e $\sigma_{X_{0}}$ em função de $X_{\max }$. Os parâmetros mostrados são referentes as funções $\mu_{X_{0}}=a_{0}+a_{1} \cdot X_{\max }+a_{2} \cdot X_{\max }^{2}$ e $\sigma_{X_{0}}=b_{0}+b_{1} \cdot X_{\max }+b_{2} \cdot X_{\max }^{2} \ldots \ldots \ldots \ldots$. . . . . . 82

Tabela 4.4 - Resultados obtidos através do método SVD. Os valores de $\left\langle X_{\max }\right\rangle$, $R M S_{X_{\text {max }}}$ e $\sigma$ estão em unidade de $\mathrm{g} / \mathrm{cm}^{2} \ldots \ldots . \ldots . . . . . . .83$

Tabela 4.5 - Resultados obtidos através do método iterativo bayesiano. Os valores de $\left\langle X_{\max }\right\rangle, R M S_{X_{\max }}$ e $\sigma$ estão em unidade de $\mathrm{g} / \mathrm{cm}^{2}$. 



\section{SUMÁRIO}

1 Introdução

2 Raios cósmicos de altas energias

2.1 Fontes, aceleração e propagação . . . . . . . . . . . . . . . . . 27

2.2 Espectro de energia . . . . . . . . . . . . . . . . . . 28

2.3 Física de partículas e os chuveiros atmosféricos . . . . . . . . . . . . . . 30

2.4 Composição . . . . . . . . . . . . . . . . . . . . . . . . 33

3 Observatório Pierre Auger 39

3.1 Detector de superfície . . . . . . . . . . . . . . . . . . . . . . . . 39

3.2 Telescópios de fluorescência $\ldots \ldots$. . . . . . . . . . . . . . . . . . 41

3.3 Reconstrução dos eventos . . . . . . . . . . . . . . . . . . . . . . . . . . . . . 42

3.3 .1 Geometria . . . . . . . . . . . . . . . . . . . . 42

3.3.2 Função de distribuição lateral . . . . . . . . . . . . . . . . . . . . . 46

3.3.3 Perfil longitudinal e energia . . . . . . . . . . . . . . . . 47

4 Taxa de elongação: métodos de deconvolução 51

4.1 Introdução . . . . . . . . . . . . . . . . . . . . 51

4.1 .1 O problema da deconvolução . . . . . . . . . . . . . . . . . 51

4.1 .2 Algoritmos de deconvolução . . . . . . . . . . . . . . . . . . . . . 54

4.1.2.1 Método SVD . . . . . . . . . . . . . . . . . . . . . . 54

4.1.2.2 Método iterativo bayesiano . . . . . . . . . . . . 58

4.2 Construção da matriz resposta . . . . . . . . . . . . . . . . . . . 59

4.3 Testes de consistência . . . . . . . . . . . . . . . . . . . . . . . . . . . . . . 62

4.4 Análise de dados . . . . . . . . . . . . . . . . . . . . . . . 63 
4.4.1 Seleção dos dados . . . . . . . . . . . . . . . . . . . . . . . . . . 63

4.4 .2 Incertezas estatísticas . . . . . . . . . . . . . . . . 65

4.4 .3 Incertezas sistemáticas . . . . . . . . . . . . . . . . . . . . 68

4.4.4 Número de iterações (somente método bayesiano) . . . . . . . . . . 72

4.5 Resultados e discussões . . . . . . . . . . . . . . . . . . . . . . . . . . . 73

5 Análise multiparamétrica: redes neurais $\quad 91$

5.1 Introdução . . . . . . . . . . . . . . . . . . . . . . . . . . . . . . . . 91

5.1 .1 Análises multiparamétricas . . . . . . . . . . . . . . . . . . . . 91

5.1 .2 Redes neurais . . . . . . . . . . . . . . . . . . . . 92

5.2 Definição dos parâmetros . . . . . . . . . . . . . . . . . . . . . . . . . . . . 94

5.3 Simulações . . . . . . . . . . . . . . . . . . . . . . . . . . . . 96

5.4 Dados medidos . . . . . . . . . . . . . . . . . . . . . . . . . . . . . . . . . . . . . . . . . . 99

5.5 Seleção dos eventos . . . . . . . . . . . . . . . . . . . . . . . . . . . . . . . . . . . . 99

5.6 Configuração, treinamento e implementação da rede neural . . . . . . . . . 103

5.7 Resultados e discussões . . . . . . . . . . . . . . . . . . . . . . . . . . . . . 104

5.7 .1 Massa nuclear . . . . . . . . . . . . . . . . . . . . . . 104

5.7 .2 Energia . . . . . . . . . . . . . . . . . . . . . 106

6 Conclusões 113

6.1 Taxa de elongação: métodos de deconvolução . . . . . . . . . . . . . . . 113

6.2 Análise multiparamétrica: redes neurais . . . . . . . . . . . . . . . . . 114

6.3 Conclusões gerais . . . . . . . . . . . . . . . . . . . . . . . . . 115

$\begin{array}{ll}\text { REFERÊNCIAS } & 117\end{array}$ 


\section{CAPÍtulo 1}

\section{Introdução}

As investigações científicas a cerca dos chamados raios cósmicos se iniciaram há aproximadamente 100 anos com os experimentos de balão do austríaco Victor Hess (1). Usando eletroscópios para medir a radiação, Hess apresentou resultados mostrando que a intensidade da radiação medida aumentava com a altitude atmosférica e portanto essa radiação era proveniente de fora do planeta, e não do solo como acreditavam os cientistas da época. A partir de então, os experimentos de raios cósmicos tornaram-se cade vez mais importantes para a construção do conhecimento científico moderno.

Atualmente existe uma geração de novos experimentos destinados a medir raios cósmicos de altíssimas energias. A energia dessas partículas pode alcançar valores da ordem de $10^{20} \mathrm{eV}$, ou seja, quatro ordens de grandeza acima das energias alcançadas nos maiores aceleradores de partículas atuais. Obter informações sobre as astropartículas nessa região de energia é fundamental para abordagem de vários problemas abertos em astrofísica de altas energias. Entre essas questões estão os processos de aceleração, a interação dessas partículas com o meio galáctico e inter-galáctico, matéria escura e campos magnéticos. No capítulo 2 são apresentadas resumidamente algumas dessas questões atuais sobre raios cósmicos de altas energias.

O conhecimento sobre a composição química dos raios cósmicos de altas energias é essencial na abordagem da maioria das questões abertas atualmente. Neste sentido, a comunidade científica esbarra no grande desafio experimental que é inferir o tipo de partícula a partir de medidas indiretas dos chamados chuveiros atmosféricos. Esses objetos são cascatas de partículas secundárias formadas após a interação da astropartícula primária com átomos da atmosfera. Existe uma série de técnicas destinadas à detecção desses chuveiros que são aplicadas nos observatórios atuais. O grande problema é que, devido a flutuações estatísticas naturais, a informação sobre a composição das partículas não pode ser extraída diretamente dos observáveis acessíveis experimentalmente. Logo, o problema deve ser abordado através de análises estatísticas mais avançadas, capazes de inferir indiretamente a composição média das partículas iniciais. O objetivo deste trabalho é estudar a composição dos raios cósmicos de altas energias através da aplicação de técnicas novas de análise aos dados medidos pelo Observatório Pierre Auger. 
Entre os muitos experimentos voltados à astrofísica de partículas, o Observatório Pierre Auger (2) se destaca principalmente por ser o maior em extensão $\left(3000 \mathrm{~km}^{2}\right)$ e por ser o primeiro a utilizar um sistema híbrido de detecção, com detectores de superfície e de fluorescência funcionando simultaneamente. Os detectores de superfície são formados por tanques de água Cherenkov que medem a frente do chuveiro no plano da superfície e os detectores de fluorescência são formados por telescópios que medem o chuveiro durante seu desenvolvimento na atmosfera. A configuração experimental do observatório foi pensada para medir partículas com energias acima de $10^{18} \mathrm{eV}$, chegando até $10^{20} \mathrm{eV}$. Extensões instaladas posteriormente alteraram o limite inferior para $10^{17.0} \mathrm{eV}$. No capítulo 3 apresentamos uma descrição resumida do processo de medido do Observatório Pierre Auger.

Neste trabalho, apresentaremos análises de composição baseadas nos parâmetros medidos pelos detectores de fluorescência. Através da emissão de radiação ultravioleta devido a interação das partículas do chuveiro com os átomos da atmosfera, os telescópios de fluorescência são capazes de medir o número de partículas da cascata em função da profundidade atmosférica. Esse é o chamado perfil longitudinal do chuveiro. O número de partículas do chuveiro tende a aumentar até uma certa profundidade e depois começa a diminuir devido à absorção de partículas pela atmosfera. A profundidade na qual o chuveiro atinge o maior número de partículas é chamado de $X_{\max }$ e é o parâmetro mais usado em análises de composição. A evolução do valor médio de $X_{\max }$ com a energia é a chamada de taxa de elongação. É possível inferir a composição média das astropartículas comparando a taxa de elongação medida por experimentos com simulações baseadas em modelos teóricos.

A análise do $X_{\max }$ realizada pela colaboração do Observatório Pierre Auger inclui os chamados cortes de fidelidade (3) nos eventos com o objetivo de garantir que os dados selecionados não estão enviesados pelo processo de medida. Um efeito colateral dessa técnica é a exclusão de um número alto de eventos e portanto a diminuição do poder estatístico do resultado. No capítulo 4 apresentamos uma análise alternativa da taxa de elongação que utiliza métodos de deconvolução para eliminar os efeitos do detector nos dados, sem a necessidade do uso dos cortes de fidelidade. Neste caso, o número de eventos disponíveis para a análise dobra em média, com relação a análise padrão.

A idéia básica dos métodos de deconvolução é extrair o efeito dos detectors em distribuições medidas por experimentos de física de altas energias, de modo a obter resultados desenviesados que podem ser comparados com resultados de outros experimentos e com modelos teóricos. Dois métodos de deconvolução foram utilizados nesta dissertação para corrigir as distribuições de $X_{\max }$ medidas pelo Observatório Pierre Auger. Foram escolhidos o método SVD (4) e o método iterativo bayesiano (5). O primeiro pertence a classe dos métodos de regularização 
e utiliza ferramentas de álgebra linear, como a chamada decomposição em valores singulares (SVD). O segundo utiliza princípios de estatística bayesiana para chegar a uma solução através de iterações.

Além do $X_{\max }$, outros parâmetros podem ser extraídos do perfil longitudinal. No capítulo 5 apresentamos uma análise inovadora que utiliza mais 2 parâmetros do perfil ( $\sigma$ e $N_{\max }$ ) em uma análise multiparamétrica de composição. A ferramenta utilizada nessa análise são as chamadas redes neurais do tipo Multilayers Perceptrons (6). Esses métodos são comumente usados para resolver problemas de inferência estatística através de um processo de aprendizagem. Isso significa, no caso deste trabalho, que a partir de um treinamento usando simulações de Monte Carlo, será possível inferir a massa média e a energia dos primários dos chuveiros medidos. No capítulo 6 faremos as conclusões e discutiremos os trabalhos futuros que devem ser realizados a partir das análises apresentadas nessa dissertação. 


\section{Raios cósmicos de altas energias}

\subsection{Fontes, aceleração e propagação}

Ainda hoje, os objetos e fenômenos astrofísicos responsáveis pela geração de raios cósmicos de altas energias continuam sendo incógnitas para a comunidade científica. Os modelos sobre a origem dessas partículas podem ser divididos em duas classes: top-down (7, 8) e bottomup (9). O primeiro caso é muito pouco aceito atualmente e prevê que as astropartículas de altíssimas energias seriam resultados do decaimento de partículas exóticas e extremamente massivas, que teriam sido produzidas no universo primordial, logo após o Big Bang. Além de hádrons, a maioria desses modelos prevêm a geração de um número alto de fótons de altas energias $\left(>10^{18} \mathrm{eV}\right)$. Entretanto, resultados experimentais recentes mostraram que o limite superior para o número de fótons de altas energias medidos é extremamente baixo, o que não favorece a aceitação desses modelos (10, 11).

A segunda classe dos modelos de geração, chamada de bottom-up, propõe mecanismos de aceleração de partículas em fontes astrofísicas. Os modelos mais aceitos dentro dessa classe, que não necessitam de teorias físicas novas, envolvem mecanismos de aceleração através de campos eletromagnéticos. O mecanismo de Fermi (12) é o mais conhecido e propõe um processo de múltiplos choques das partículas com campos magnéticos em movimento como responsável pela transferência de energia. Entre os objetos que poderiam acelerar partículas pelo mecanismo de Fermi estão os remanescentes de supernovas, núcleos ativos de galáxia (AGN) (13) e estrelas de neutrôns (14).

Existem várias propriedades dos objetos astrofísicos que limitam suas capacidades em acelerar partículas altamente energéticas. As principais estão relacionadas as dimensões e a intensidade dos campos magnéticos de cada objetos. Com o objetivo de avaliar, através dessas propriedades, quais seriam as possíveis fontes de raios cósmicos de altas energias, Hillas propôs (13) uma cálculo aproximado para a energia máxima $\left(E_{\max }\right)$ que poderia ser transferida à partículas com número atômico $Z$, considerando o campo magnético $B$ e a dimensão da fonte $R$. Simplificadamente temos que $E_{\max } \sim Z B R$. O gráfico mostrado na figura 2.1 é 


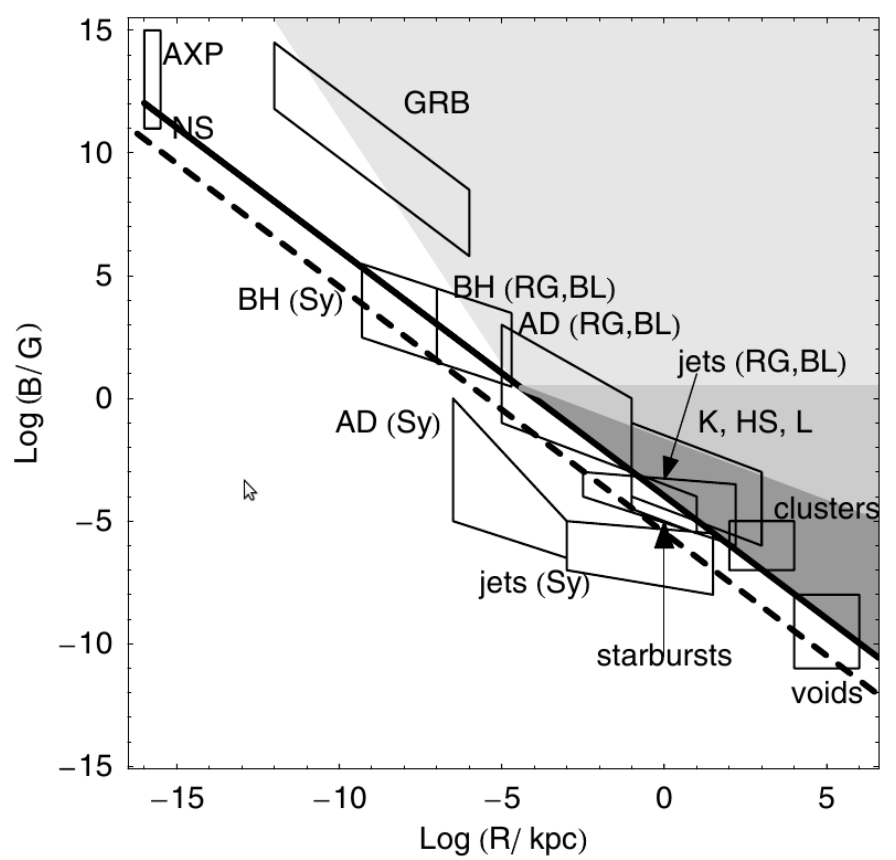

Figura 2.1 - Diagrama de Hillas mostrando possíveis fontes astrofísicas capazes de acelerar raios cósmicos. Fonte: Imagem extraída da referência (15).

conhecido como "diagrama de Hillas" e mostra as fontes astrofísicas conhecidas em função da dimensão e do campo magnético e os limites máximos de energia de aceleração.

Além dos processos de aceleração, os efeitos da propagação também são importantes e podem gerar assinaturas nas grandezas observadas pelos experimentos. A deflexão pelos campos magnéticos é um dos principais efeitos que influenciam a propagação das partículas carregadas. Para o caso de núcleos, a fotodesintegração também é muito importante em altas energias e consiste na desintegração de núcleos, gerando núcleos menores, através de interação com um fóton. Para prótons, a interação com fótons é responsável pelo fotoprodução de píons.

\subsection{Espectro de energia}

O fluxo de raios cósmicos (ou seja, o número de partículas medidas por unidade de tempo, de área e de ângulo sólido) em função da energia é chamado de espectro de energia. A figura 2.2 mostra o espectro de raios cósmicos medidos por vários experimentos entre $10^{8}$ e $10^{20} \mathrm{eV}$. O comportamento observado no espectro é aproximadamente linear e portanto, o fluxo de raios cósmicos pode ser descrito como uma lei de potência do tipo $\frac{d N}{d E} \sim E^{-\gamma}$, sendo $\gamma$ o chamado índice espectral. 


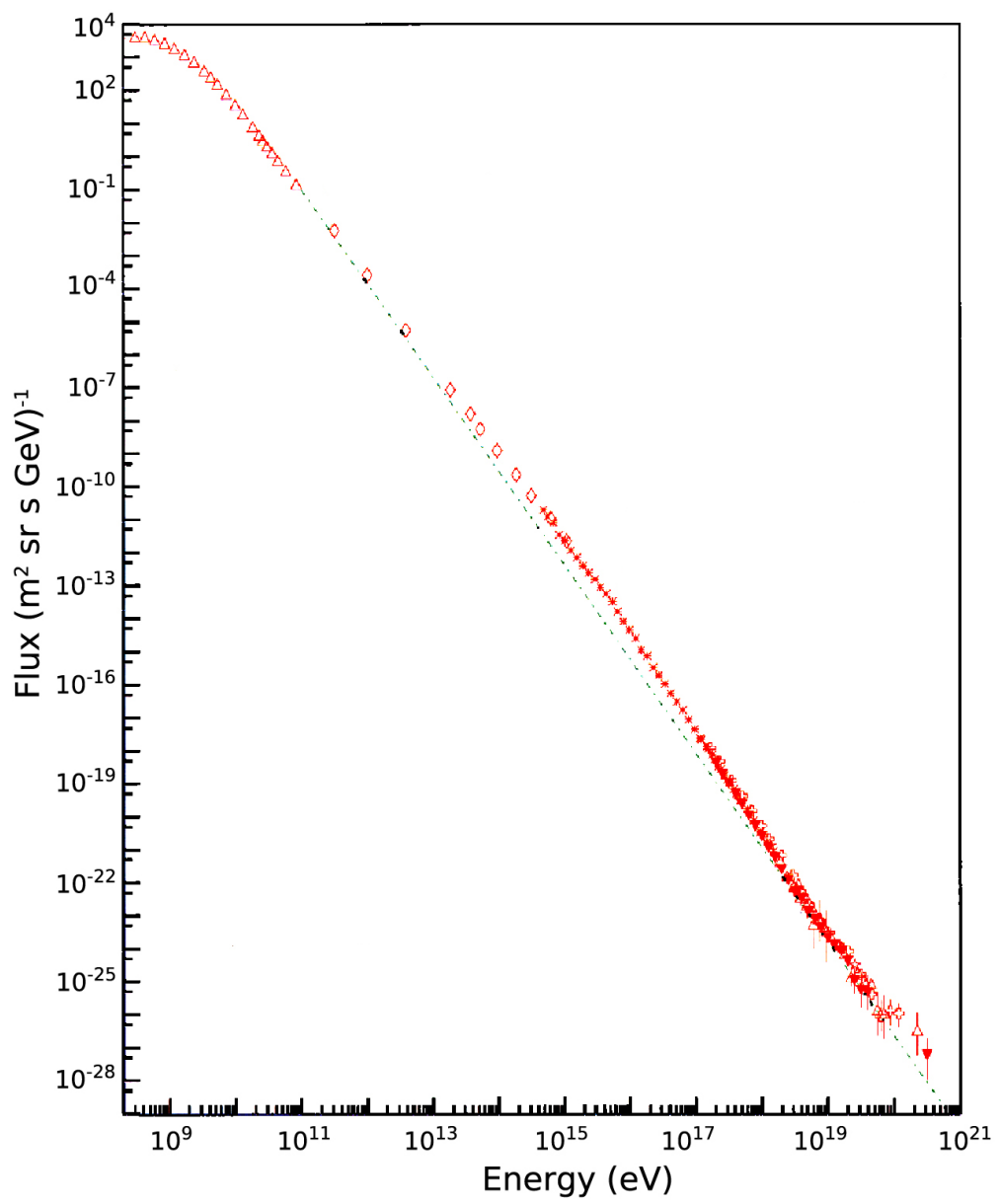

Figura 2.2 - Espectro de energia dos raios cósmicos. Fonte: Imagem disponível em http://www.perdaix.de/cosmic_radiation. 
$\mathrm{O}$ valor de $\gamma$ é de 2.7 abaixo de aproximadamente $5 \times 10^{15} \mathrm{eV}$, e nessa energia se altera para 3.0. Esta primeira quebra no fluxo é chamada de "joelho" (16). Entre os vários modelos destinados a explicar os fenômenos envolvidos nesta estrutura, os mais creditados propõem um limite de aceleração que depende da carga da partícula acelerada. Em $5 \times 10^{15} \mathrm{eV}$ esta quebra pode ser bem descrita pelo limite em energia da aceleração de próton. Uma segunda quebra, medida em aproximadamente $8 \times 10^{16} \mathrm{eV}$, neste modelo corresponderia ao limite em energia para aceleração de núcleos de ferro e é chamada de "segundo joelho" (17).

A segunda estrutura significativa no espectro ocorre aproximadamente em $3 \times 10^{18} \mathrm{eV}$, onde o índice espectral muda novamente de 3.0 para 2.7 e é chamada de "tornozelo" (18). Os modelos mais aceitados para explicar essa estrutura propõem que ocorra a transição entre raios cósmicos galácticos e extragalácticos nesta região de energia. Porém, existem ainda muitos outros modelos propondo diferentes motivos para a existência desta quebra. A figura 2.3 mostra o espectro de energia medido por vários experimentos na região de altíssimas energias, contendo a região do "tornozelo". Outra característica muito importante do espectro é o chamado "corte GZK", que é uma supressão no fluxo de raios cósmicos acima de aproximadamente $5 \times 10^{19} \mathrm{eV}$. Esse corte foi proposto por Greisen (19), Katsepin e Kuz' min (20) e seria originado pela interação das astropartículas com os fótons da radiação cósmica de fundo. Pela figura 2.3 pode-se notar que existe um corte na região do GZK nos dados do Observatório Pierre Auger (21) e do HiRes (22), porém não aparece nos dados do AGASA (23). A real causa da supressão no espectro observada nos dados do Auger e do HiRes ainda é desconhecida. Além do corte GZK, o limite natural de aceleração de algum tipo de fonte pode ser responsável por tal efeito.

\subsection{Física de partículas e os chuveiros atmosféricos}

Além das questões astrofísicas, os raios cósmicos têm sido muito importantes ao longo das história no estudo da física de partículas. Até o início da construção dos aceleradores, os raios cósmicos eram a principal fonte para descobertas de novas partículas. Atualmente, a atenção da comunidade científica está direcionada às interações de altíssimas energias que acontecem entre as astropartículas e os átomos da atmosfera. O estudo destas interações é possível somente a partir do entendimento sobre o desenvolvimento dos chuveiros atmosféricos.

Os chamados chuveiros atmosféricos são cascatas de partículas formadas a partir da interação de uma astropartícula de alta energia com átomos do topo de nossa atmosfera (25). 0 número de partículas secundárias que constituem essa cascata é extremamente alto, chegando 


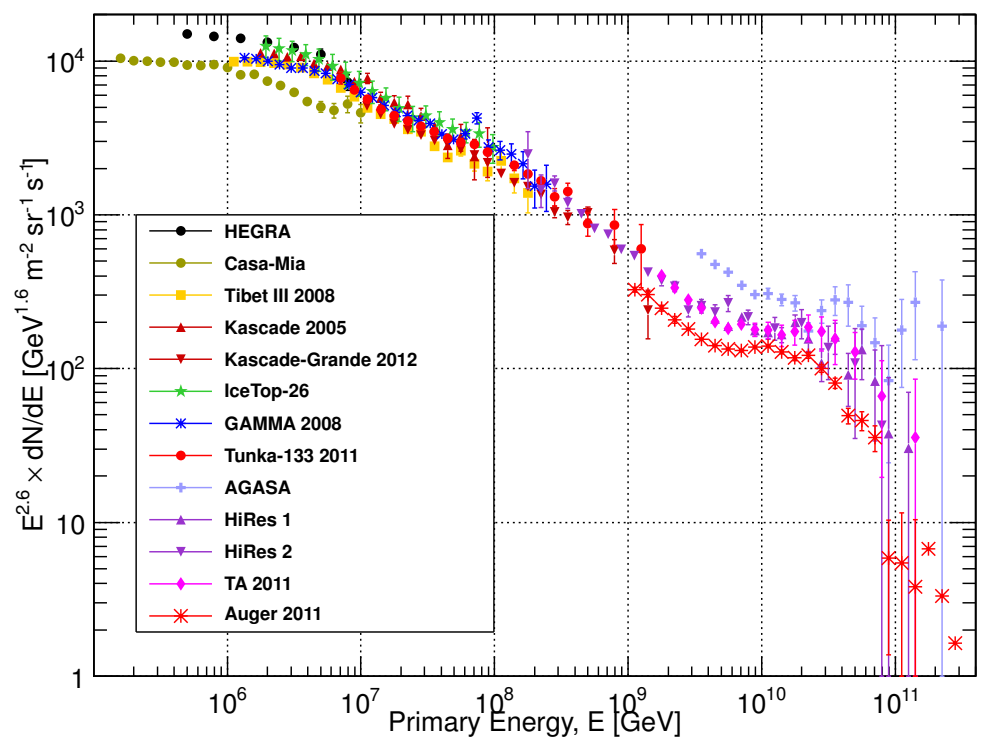

Figura 2.3 - Espectro de energia dos raios cósmicos acima de $10^{14.0} \mathrm{eV}$. O fluxo está multiplicado por $E^{2.6}$ para facilitar a visualização das estruturas. Fonte: Imagem extraída da referência (24).

à $10^{10}$ em um chuveiro iniciado por um primária de energia $10^{18} \mathrm{eV}$. A extensão da frente de partículas pode chegar a ter dezenas de $\mathrm{km}^{2}$. Dado que o fluxo de astropartículas é extremamente baixo no regime de altíssimas energias, a detecção direta destas é inviável experimentalmente e portanto a medição indireta através dos chuveiros atmosféricos é fundamental para o estudo desses raios cósmicos de altas energias.

A interação inicial responsável pela formação dos chuveiros acontece na colisão da partícula incidente com um núcleo de algum átomo da atmosfera, e portanto essa interação é de natureza essencialmente hadrônica. Como resultado dessa primeira colisão são formados principalmente núcleos secundários, píons carregados $\left(\pi^{+}\right.$e $\left.\pi^{-}\right)$e píons neutros $\left(\pi^{0}\right)$. Os núcleos secundários colidem em seguida com outros núcleos iniciando outras cascatas secundárias enquanto os píons decaem em outras partículas $\left(e^{+}, e^{-}, \nu, \gamma\right.$ e $\mu$ ) dando continuidade a formação do chuveiro. O conjunto de píons, núcleos secundários e outros hádrons formados no início do desenvolvimento do chuveiro constituem a chamada componente hadrônica. A extensão dessa parte da cascata é muito reduzida, uma vez que o tempo de vida dos píons é muito curto e seus decaimentos são responsáveis pela formação das outras duas componentes do chuveiro, a eletromagnética e a muônica.

Os decaimentos dos píons carregados que constituem a componente hadrônica dos chuveiros geram como resultado múons $\left(\mu^{+}\right.$e $\left.\mu^{-}\right)$, elétrons/pósitrons $\left(e^{-}\right.$e $\left.e^{+}\right)$e neutrinos $(\nu)$. Esse múons são muito estáveis e tem probabilidade de interação muito baixa ao longo do 


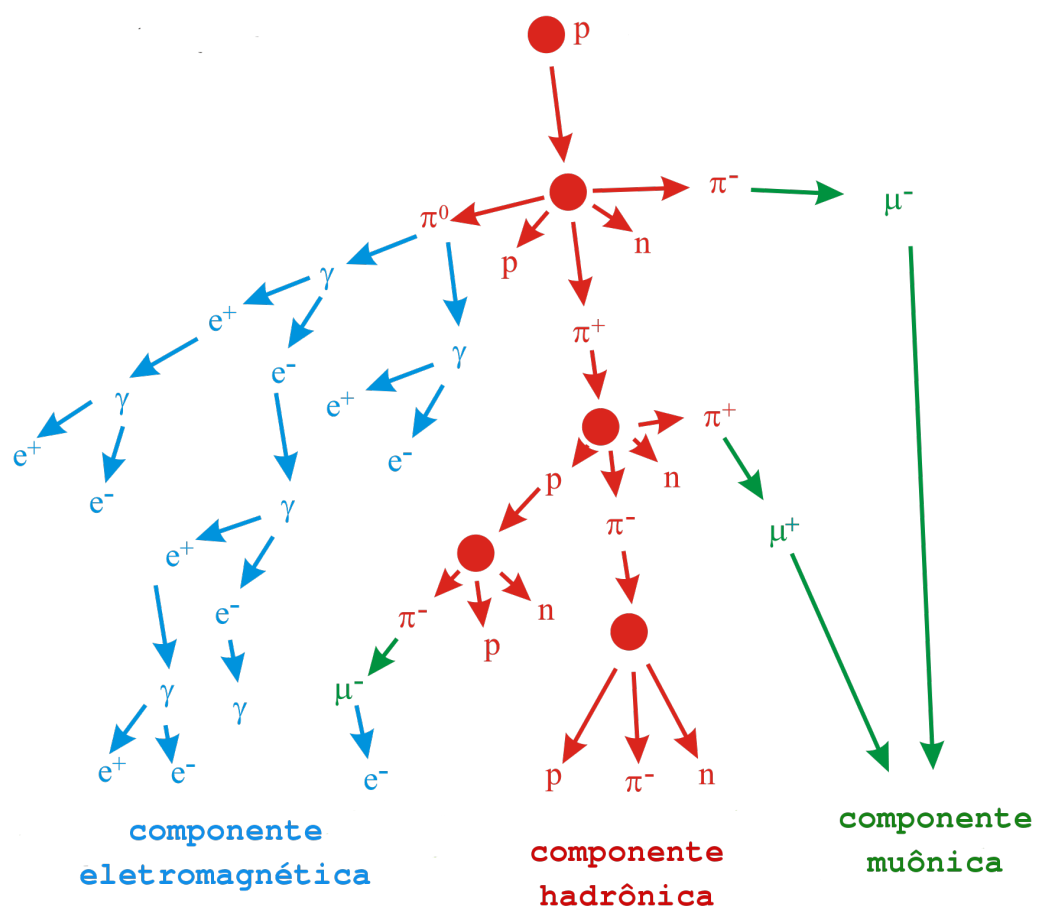

Figura 2.4 - Esquema do desenvolvimento de um chuveiro atmosférico. Fonte: Imagem disponível em http://commons.wikimedia.org/wiki/File:AirShower.svg.

restante da atmosfera. Portanto, o número de múons que constituem a componente muônica do chuveiro cresce durante um intervalo de seu desenvolvimento em que estas partículas são intensamente produzidas e depois permanece praticamente constante até atingir a superfície.

A componente eletromagnética dos chuveiros é iniciada principalmente pelos fótons $(\gamma)$ gerados pelos decaimentos dos píons neutros $\left(\pi^{0}\right)$. Esses fótons geram elétrons e pósitrons através da chamada produção de pares, e esses geram mais fótons através do processo chamado de bremsstrahlung, e assim os processos continuam sucessivamente. A energia contida na componente eletromagnética é muito maior que para as outras duas componentes (hadrônica e muônica), sendo da ordem de $90 \%$ da energia total do chuveiro. O número de partículas neste caso, ao contrário do caso dos múons, cresce até um certo limite em que a energia das partículas secundárias é baixa e portanto a probabilidade de absorção pela atmosfera é comparável à probabilidade de geração de novas partículas. A partir deste limite o número de partículas começa a diminuir. As medições do desenvolvimento longitudinal dos chuveiros é baseado na interação das partículas secundárias com as moléculas da atmosfera. A figura 2.4 mostra um esquema do desenvolvimento de um chuveiro atmosférico destacando as três componentes descritas acima.

As medidas do perfil são feitas geralmente em função da profundidade atmosférica, que é 


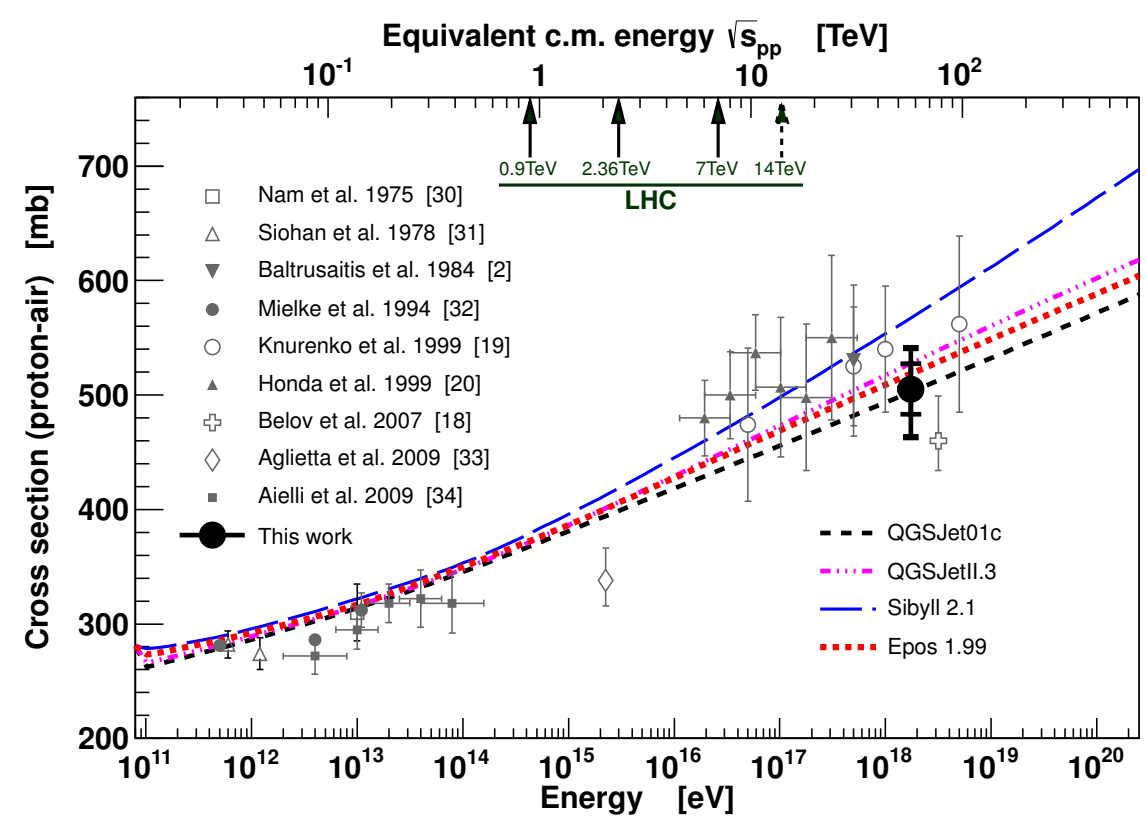

Figura 2.5 - Seção de choque próton-ar medida por vários experimentos e obtidas através de modelos teóricos. Fonte: Imagem extraída da referência (26).

dada por,

$$
X(h)=\int_{h}^{\text {infinito }} \frac{\rho\left(h^{\prime}\right) d h^{\prime}}{\cos \theta},
$$

em que $h$ é a altitude atmosférica, $\rho$ é a densidade da atmofera e $\theta$ é o ângulo zenital do eixo do chuveiro. Nota-se pela equação 2.1 que a grandeza $X$ é uma medida da quantidade de matéria atravessada pelo chuveiro e não apenas de um intervalo de distância de seu desenvolvimento. A profundidade atmosférica em que o chuveiro alcança o maior número de partículas é chamado de $X_{\max }$ e é um parâmetro muito importante para estudos de composição de raios cósmicos.

A partir das ditribuições de $X_{\max }$ também é possível extrair informações sobre a interação inicial responsável pela formação do chuveiro. Mais precisamente, é possível calcular a seção de choque próton-ar. Recentemente a colaboração do Observatório Pierre Auger publicou resultados desse cálculo (26). A figura 2.5 mostra o valor da seção de choque calculado em comparação com outros experimentos e com modelos teóricos.

\subsection{Composição}

Estudar a composição de raios cósmicos a partir da medição dos chuveiros atmosféricos exige o entendimento sobre os fenônemos físicos envolvidos no desenvolvimento destas cascatas. Entretanto, algumas diferenças importantes entre os chuveiros formados por primários 
com massas diferentes podem ser extraídas de modelos simples. O principal desses diz que um núcleo de massa $A$ e energia $E$ pode ser tratado aproximadamente como $A$ prótons (ou neutrons) de energia $E^{\prime}=E / A$. Esse é o chamado modelo de superposição (27). Apesar de aproximado, esse modelo é capaz de prever muito bem diferenças qualitativas quanto ao desenvolvimentos dos chuveiros. Descrições mais elaboradas são usadas pelos códigos de transporte usados nas simulações. Entre eles estão CORSIKA (28), AIRES (29) e CONEX (30, 31).

A evolução do $X_{\max }$ com a energia é a maneira mais direta de inferir a composição dos primários. Neste sentido, defini-se a grandeza $D=d\left\langle X_{\max }\right\rangle / d(\ln E)$, chamada de taxa de elongação*. A partir do conhecimento sobre os principais fenômenos envolvidos no desenvolvimento dos chuveiros é possível mostrar que para chuveiros iniciados por prótons temos,

$$
\left\langle X_{\max }^{p}\right\rangle=c+D_{p} \ln E
$$

em que $c$ é uma constante. Nesse caso $D_{p}$ é aproximadamente constante e depende das características das interações hadrônicas envolvidas no início da formação do chuveiro. Considerando agora o modelo de superposição, obtemos para chuveiros iniciados por núcleos de massa $A$ que,

$$
\left\langle X_{\text {max }}^{A}\right\rangle=c+D_{p} \ln (E / A)=c-D_{p} \ln A+D_{p} \ln E .
$$

Se considerarmos agora a média do $X_{\max }$ de chuveiros iniciados por diferentes primários temos que,

$$
\left\langle X_{\max }\right\rangle \approx \sum_{i} f_{i}\left\langle X_{\max }^{A_{i}}\right\rangle=\left\langle X_{\max }^{p}\right\rangle-D_{p}\langle\ln A\rangle
$$

em que $f_{i}$ é a fração de chuveiros iniciados por cada núcleo de massa $A_{i}$. Desta relação podemos extrair a seguinte dependência de $\langle\ln A\rangle \operatorname{com}\left\langle X_{\max }\right\rangle$ para uma dada energia,

$$
\langle\ln A\rangle=\frac{\left\langle X_{\max }^{p}\right\rangle-\left\langle X_{\max }\right\rangle}{\left\langle X_{\max }^{p}\right\rangle-\left\langle X_{\max }^{F e}\right\rangle} \ln 56 .
$$

O valor de $D_{p}$ calculado a partir de simulações é da ordem de $25 \mathrm{~g} / \mathrm{cm}^{2}$. Se considerarmos a evolução de $\left\langle X_{\max }\right\rangle$ por década de energia temos que $D_{10}^{p}=\ln 10 \cdot D_{p} \approx 60 \mathrm{~g} / \mathrm{cm}^{2}$. Logo, o $\left\langle X_{\max }\right\rangle$ de chuveiros iniados por prótons e por núcleos de ferro devem diferir de $D_{p}(\ln 56-\ln 1) \approx 100 \mathrm{~g} / \mathrm{cm}^{2}$. Notamos também que medidas experimentais da taxa de elongação não constante implica necessariamente em mudança de composição.

A figura 2.6 mostra o $\left\langle X_{\max }\right\rangle$ medido por vários experimentos comparado com simulações.

* Neste texto o termo taxa de elongação será também utilizado em outros sentidos, como por exemplo para se referir ao gráfico de $\left\langle X_{\max }\right\rangle \times \log (E)$. Este abuso de linguagem é tradicional em trabalhos sobre o assunto. 
Existem dois tipos de detectores que podem medir o $X_{\text {max }}$ usados pelos experimentos citados na figura 2.6: telescópios Cherenkov (Tunka (32), Yakutsk (33) e CASA-BLANCA (34)) e de fluorescência (HiRes-MIA (35), HiRes (36), Telescope Array (37) e Observatório Pierre Auger (38)). Os dados do HiRes e do Telescope Array são publicados sem correções com relação aos efeitos do detector. Isso significa que existe um viés experimental nos valores de $\left\langle X_{\max }\right\rangle$ medido por esses observatórios. Os dados apresentados aqui foram extraídos da referência (27) em que é realizada uma correção aproximada para tornar os resultados comparáveis com os de outros experimentos.

Comparando os valores de $\left\langle X_{\max }\right\rangle$ com as simulações de prótons e núcleos de ferro, é possível notar que para energias próximas de $10^{15} \mathrm{eV}$ (região do "joelho" do espectro) a massa das partículas tende a ser leve, em média. Na região próxima a $10^{17} \mathrm{eV}$ ("segundo joelho" do espectro) o comportamento é diferente e mostra um composição mais pesada em média. Acima de $10^{18} \mathrm{eV}$ a composição é novamente leve. No regime de altíssimas energias(> $10^{19} \mathrm{eV}$ ) existe significativa discordância entre diferentes experimentos. Os resultados do HiRes, Telescope Array e Yakutsk mostram uma tendência a composição constantemente leve e o Observatório Pierre Auger mostra uma tendência a transição para partículas mais pesadas. 0 poder estatístico dos resultados do Observatório Pierre Auger é extremamente mais elevado que os outros experimentos.

Além do valor médio do $X_{\max }$, sua variância também é sensível a composição primária. A figura 2.7 mostra resultados do Yakutsk, HiRes e Auger. O gráfico da esquerda mostra - $R M S_{X_{\text {max }}}$ medido pelo Yakutsk e pelo Auger comparados com simulações de prótons e núcleos de ferro. O comportamento observado é coerente com o da figura 2.6 em termos de composição, pois os dados do Auger mostram uma transição de partículas leves para pesadas enquanto os dados do Yakutsk mostram uma composição leve constante. O gráfico da direita mostra a variância da função gaussiana ajustada às distribuições truncadas de $X_{\max }$ medidos pelo HiRes. Essa medida é análoga a medida do $R M S_{X_{\max }}$. Nesse caso novamente o mesmo comportamento do $\left\langle X_{\max }\right\rangle$ é observado: composição leve praticamente constante.

Outros resultados sobre composição podem ser obtidos por experimentos que utilizam detectores de superfície capazes de medir as componentes eletromagnética e muônica das frentes dos chuveiros. Os exemplos mais importantes dessa classe de observatórios são o KASCADE (39) e sua extensão, o KASCADE-Grande (40). Através de cintiladores blindados e não-blindados, os detectores de superfície medem o número de múons e o número de partículas carregadas, respectivamente. Com essas grandezas medidas e através da aplicação de métodos de deconvolução, é possível obter os especros de energia de diferentes tipos de partículas primárias (41). A figura 2.8 mostra o espectro total medido pelo KASCADE-Grande 


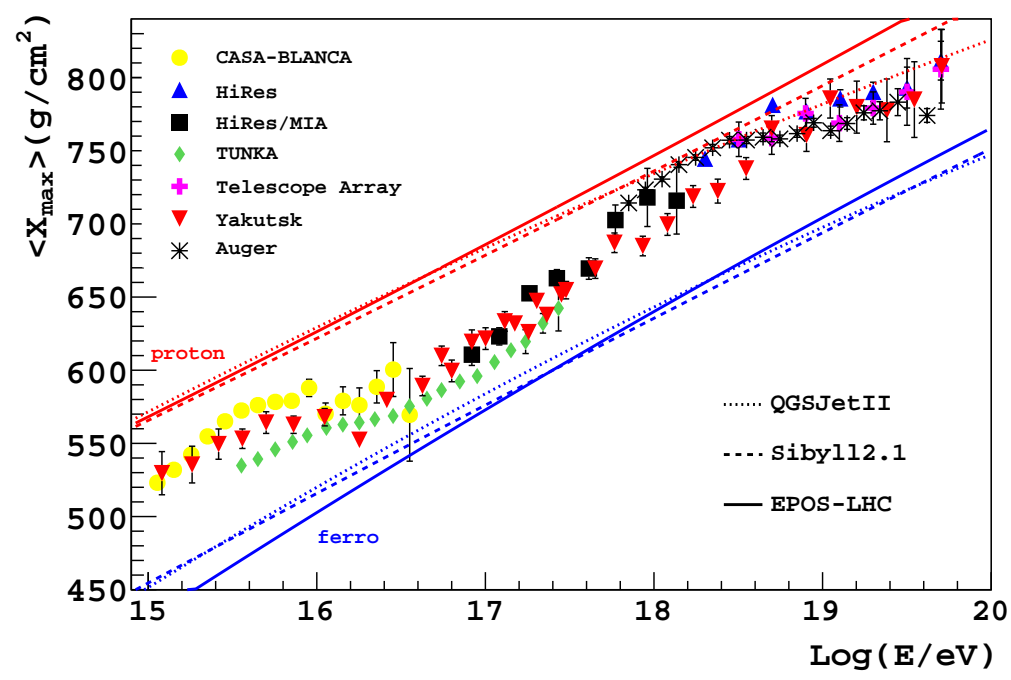

(a)

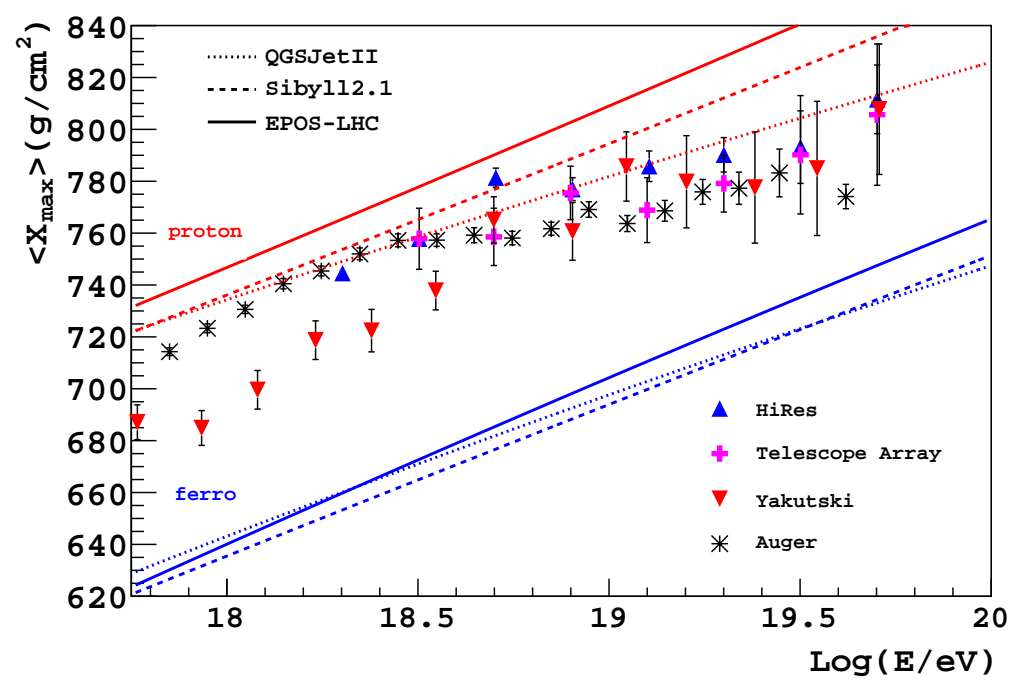

(b)

Figura 2.6 - Medidas de $\left\langle X_{\max }\right\rangle$ em função da energia para vários experimentos entre (a) $10^{15.0} \mathrm{e}$ $10^{20.0} \mathrm{eV}$ e (b) $10^{17.8}$ e $10^{20.0} \mathrm{eV}$. Os dados foram extraídos da referência (27). Fonte: Imagem elaborada pelo autor. 

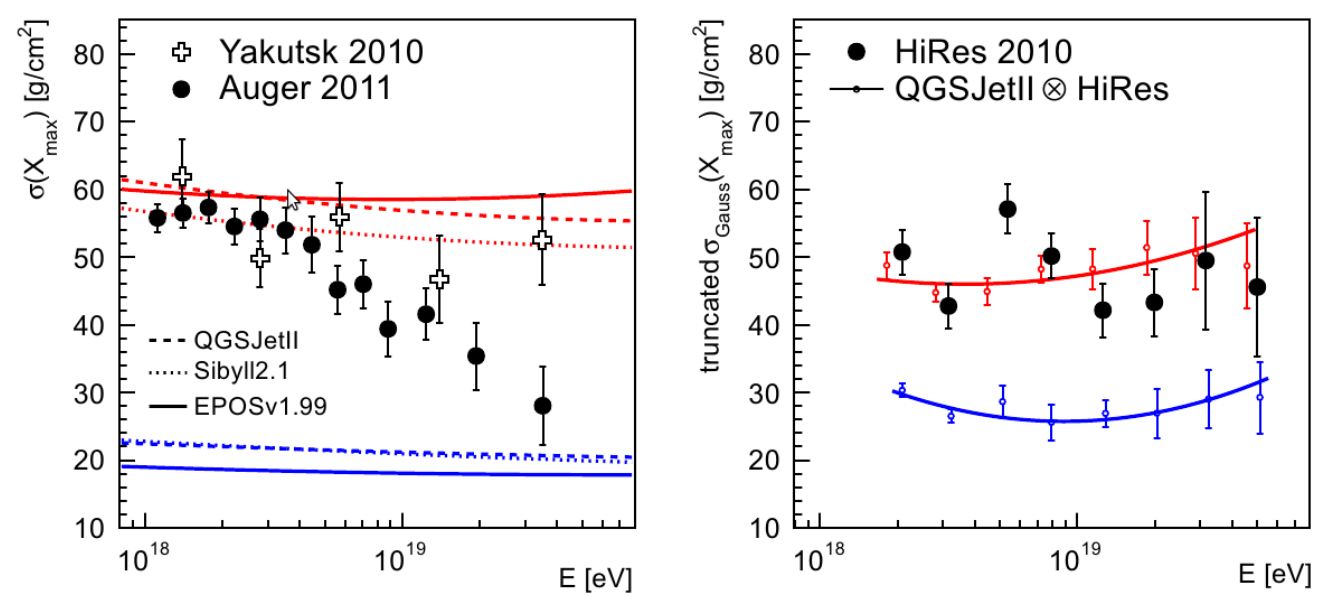

Figura 2.7 - Gráficos do $R M S$ do $X_{\max }$ (esquerda) e da variância da gaussiana ajustada às distribuições de $X_{\max }$ (direita). Fonte: Imagem extraída da referência (27).

juntamente com o espectro das componentes de partículas leves e pesadas. O intervalo de energia compreendido inclui a região do "segundo joelho" e vai de $10^{16.6}$ à $10^{18.2} \mathrm{eV}$. Como já havia sido observado na análise do $X_{\max }$, em torno de $10^{17} \mathrm{eV}$ há um predomínio de partículas pesadas em relação às partículas leves.

Existem ainda parâmetros alternativos extraídos dos chuveiros que têm sido utilizados no estudo de composição. Um dele é o chamado profundidade de produção de múons (MPD ${ }^{\dagger}$ ) (44, 45). Esse parâmetro é extraído do perfil longitudinal da produção de múons, que pode ser obtida através dos detectores de superfície. A colaboração do Observatório Pierre Auger já publicou análises baseadas no MPD, como pode ser visto na referência (46). Recentemente um novo parâmetro, chamado rise time asymmetry também tem sido estudado pela colaboração do Observatório Pierre Auger como possível parâmetro sensível a composição (47, 48). Em ambos os casos as análises são realizadas apenas na região de energias acima de $10^{19} \mathrm{eV}$ e os resultados mostram que as partículas têm uma tendência a serem mais pesadas em média nessa região do espectro. É importante notar que estas conclusões são dependentes das simulações do detectores e dos modelos de interação hadrônica.

${ }^{\dagger}$ MPD é a sigla em inglês para Muon Production Depth. 


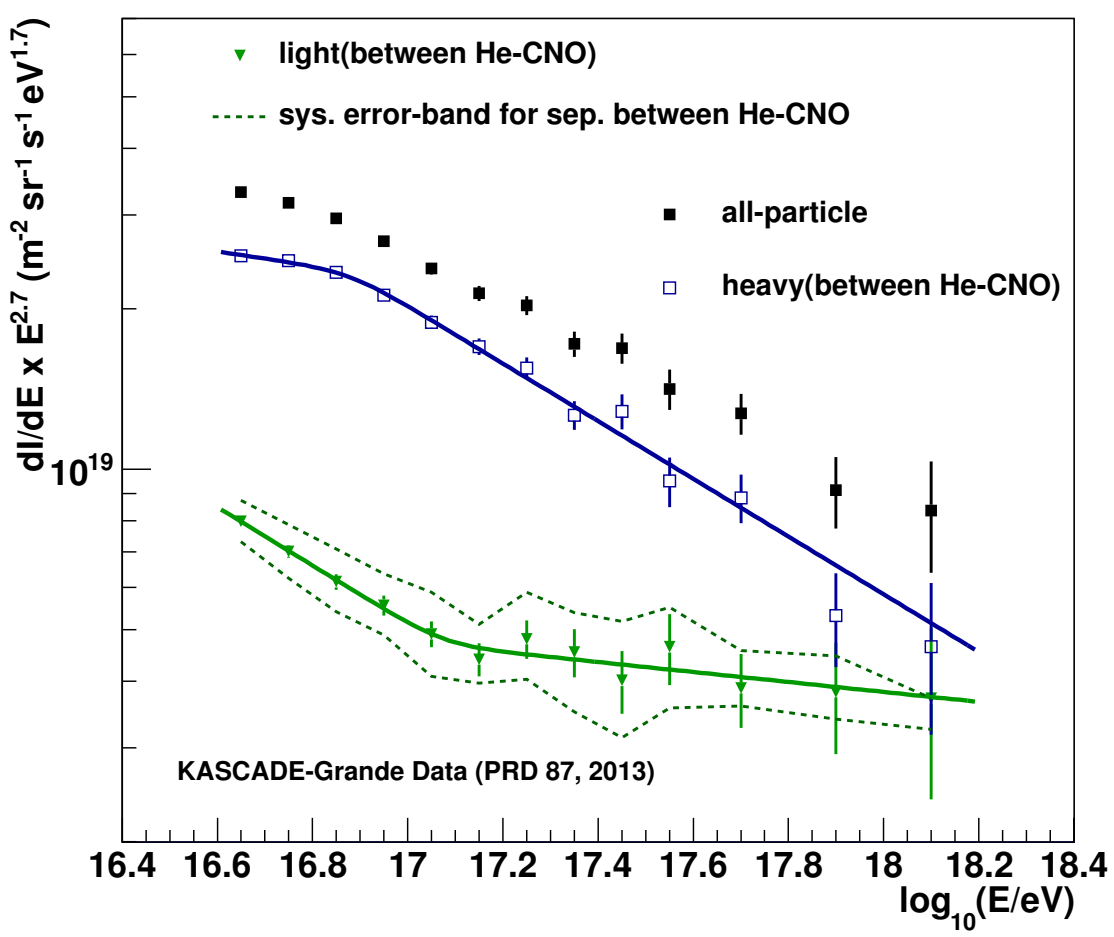

Figura 2.8 - Espectro de energia medido pelo KASCADE-Grande (40). Os dados podem ser encontrados na referência (42). Fonte: Imagem extraída da referência (43). 


\section{Observatório Pierre Auger}

O Observatório Pierre Auger está localizado próximo a cidade de Malargue, província de Mendoza, Argentina (49), e é administrado por uma colaboração formada por mais de 350 pesquisadores de 17 países, incluindo o Brasil. O objetivo principal do observatório é medir astropartículas com energias acima de $10^{19} \mathrm{eV}$, região do espectro muito pouco explorada por experimentos anteriores. Para isso foi desenvolvido pioneiramente um sistema de detecção híbrido, que conta com detectores de superfície e detectores de fluorecência operando conjuntamente. Os detectores de superfície medem a distribuição lateral dos chuveiros e este tipo de detector tem sido extensivamente utilizados ao longo das últimas décadas por outros experimentos, como o Volcano Ranch (50), AGASA (51) e o Haverah Park (52). Os telescópios de fluorescência, desenvolvidos para medir o desenvolvimento longitudinal do chuveiro na atmosfera, foram utilizados pela primeira vez pelo Fly's Eye (53) a partir do ano 1981, dando origem posteriormente ao HiRes (54) no ano de 1994. A ideia de aplicar os dois métodos de detecção conjuntamente é motivada pelo consequente aumento na precisão das medidas da direção, energia e outros parâmetros da partícula primária. Além do Observatório Pierre Auger, atualmente existe outro experimento com sistema de detecção híbrida capaz de medir partículas com energias acima de $10^{19} \mathrm{eV}$, o Telescope Array (55).

A figura 3.1 mostra um esquema da disposição dos detectores de superfície e dos telescópios de fluorescência do Observatório Pierre Auger. Nas próximas seções, serão descritos o funcionamento dos dois métodos de detecção e também o processo de reconstrução dos chuveiros.

\subsection{Detector de superfície}

O detector de superfície do Observatório Pierre Auger é constituído por um arranjo de estações que detectam as partículas da frente do chuveiro. Cada uma das estações contém 12000 litros de água ultra pura, dentro de um reservatório cilíndrico de $1.2 \mathrm{~m}$ de altura e $3.6 \mathrm{~m}$ de diâmetro (figura 3.2). As partículas do chuveiro atravessam as estações e por terem 


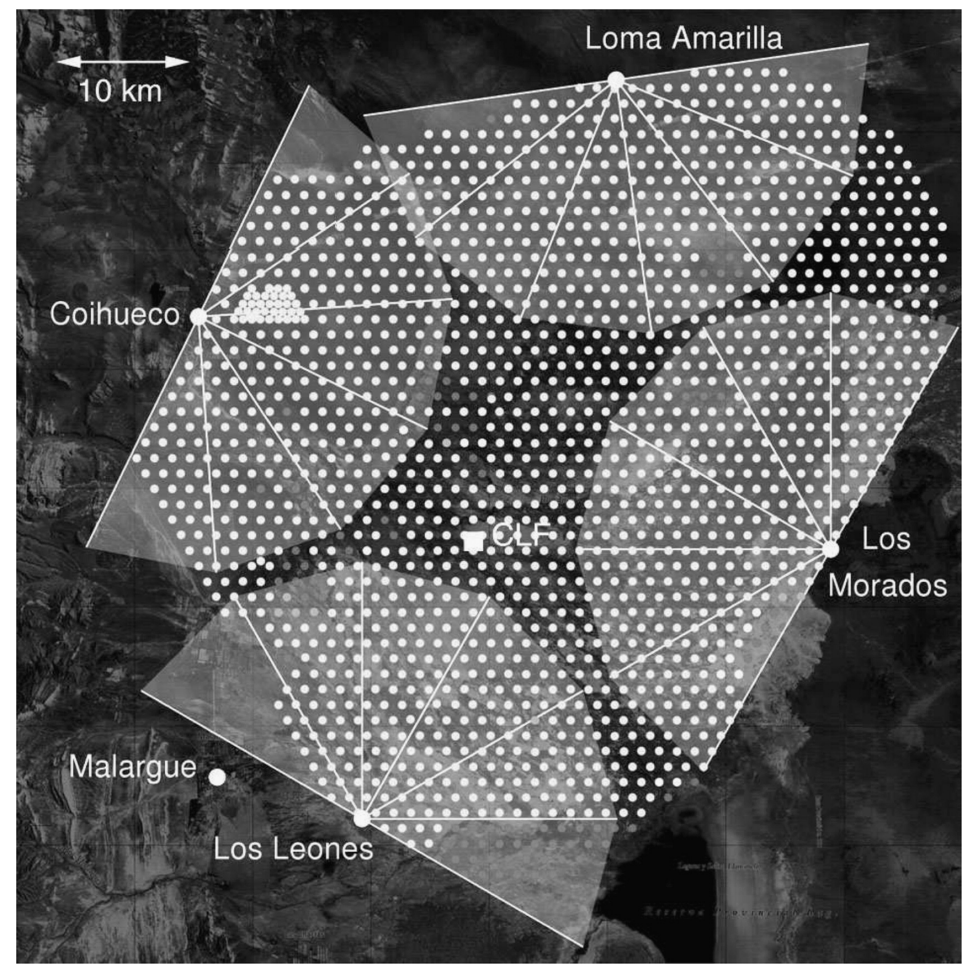

Figura 3.1 - Configuração do Observatório Pierre Auger em março de 2009. Os pontos cinza representam as posições dos detectores de superfície e a linhas indicam o campo de visão de cada um dos telescópios de fluorescência. Fonte: Imagem extraída da referência (56).

velocidade maior que a velocidade da luz na água, geram radiação Cherenkov que é detectada por três fotomultiplicadoras.

O número total de estações é de mais de 1600, e estas estão configuradas segundo um arranjo triangular com separação de $1500 \mathrm{~m}$ entre cada detector, cobrindo uma área de aproximadamente $3000 \mathrm{~km}^{2}$. Detalhes sobre a instrumentação e sobre a performance dos detectores de superfície podem ser encontradas nas referências (2, 57,-59).

O principal objetivo dos detectores de superfície é medir a chamada função de distribuição lateral (LDF*), ou seja, medir a quantidade de partículas em função da distância ao eixo do chuveiro. A partir da LDF é possível calcular o sinal das estações em uma distância particular (1000 m no caso do Observatório Pierre Auger) e com isso determinar a energia da partícula primária. Ao contrário dos telescópios de fluorescência, os detectores de superfície funcionam eficientemente mesmo durante o dia, e portanto seu ciclo de funcionamento é bem próximo de $100 \%$. O arranjo das estações foi configurado de modo que a eficiência de trigger seja de $100 \%$ para chuveiros com energia acima de $3 \times 10^{18.0} \mathrm{eV}(59,60)$. 


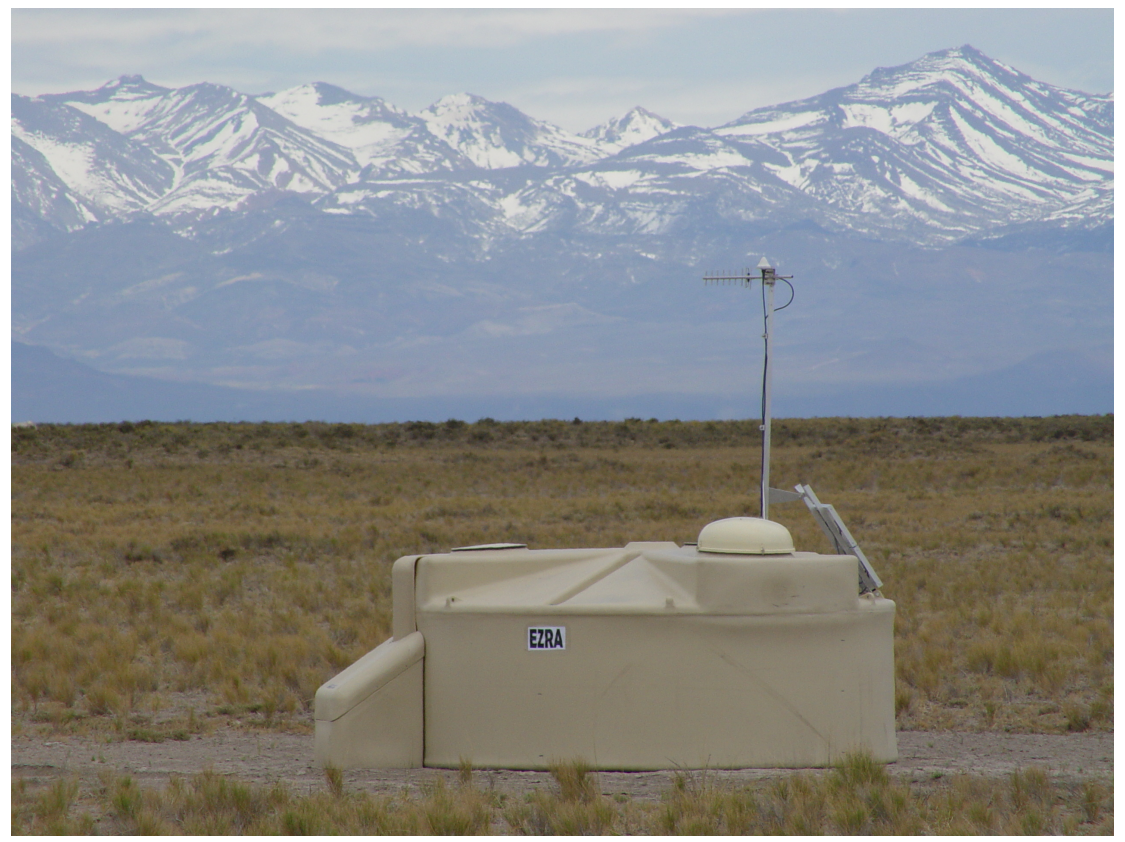

Figura 3.2 - Foto de uma das estações do detector de superfície do Observatório Pierre Auger. Fonte: Imagem disponível em http://www.auger.org/media/image_highlights.html.

\subsection{Telescópios de fluorescência}

Os telescópios de fluorescência instalados no Observatório Pierre Auger tem como objetivo medir o desenvolvimento longitudinal dos chuveiros através da radiação gerada pela interação das partículas carregadas com as moléculas da atmosfera. As partículas carregadas do chuveiro excitam as moléculas do gás nitrogênio do ar que ao voltarem ao estado fundamental emitem radiação ultravioleta $(\sim 300-430 \mathrm{~nm})$.

Atualmente estão instalados 27 telescópios divididos em 4 sítios (Los Leones, Loma Amarilo, Los Morados e Coihueco). Inicialmente foram instalados 6 telescópios em cada sítio, tendo $30^{\circ}$ de abertura azimutal cada, totalizando uma abertura de $180^{\circ}$. A elevação destes telescópios foi definida de modo que pudessem medir a partir de $0^{\circ}$ até $30^{\circ}$ de abertura zenital. A figura 3.1 mostra a disposição dos prédios e o campo de visão de cada um dos 24 telescópios iniciais. No ano de 2009 foram instalados mais 3 telescópios em Coihueco com abertura zenital de $30^{\circ}$ até $60^{\circ}$. Esta extensão é chamada de $\mathrm{HEAT}^{\dagger}$ (61) e tem como objetivo de medir chuveiros formados por partículas menos energéticas, entre $10^{17.0}$ e $3 \times 10^{18.0} \mathrm{eV}$. A figura 3.3 mostra um esquema dos telescópios de fluorescência e uma foto. A descrição detalhada dos detectores de fluorescência do Observatório Pierre Auger pode ser encontrada nas referências (2. 56).

\footnotetext{
${ }^{\dagger}$ High Elevation Auger Telescopes
} 


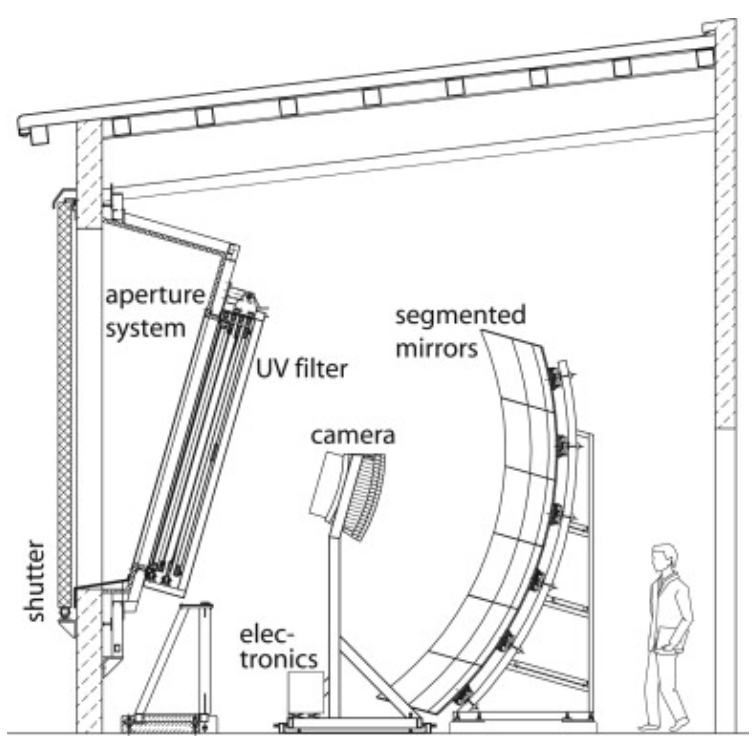

(a)

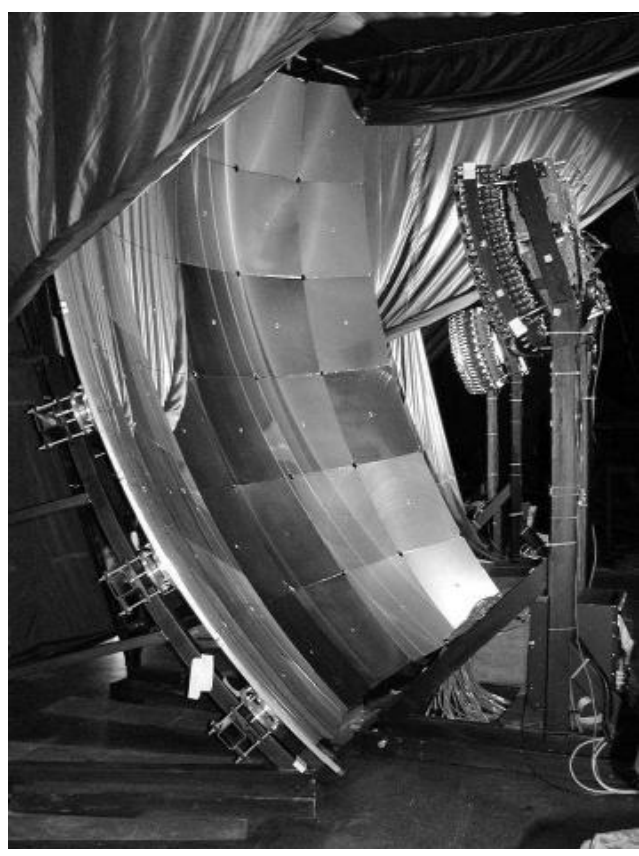

(b)

Figura 3.3 - (a) Desenho de um dos telescópios de fluorescência. Fonte: Imagem extraída da referência (56). (b) Fotografia de um dos telescópios de fluorescência do Observatório Pierre Auger. Fonte: Imagem disponível em http://www.auger.org/observatory/gallery2005.html.

A quantidade de radiação emitida pelas moléculas de nitrogênio é proporcional à energia depositada através de interações eletromagnéticas pelos chuveiros na atmosfera. A taxa em que essa energia é depositada em função da profundidade atmosférica é uma função chamada de perfil longitudinal do chuveiro, cuja integral dá a energia total dissipada eletromagneticamente, ou também chamada, energia calorimétrica. Devido à alta sensibilidade, os telescópios de fluorescência podem funcionar somente durante noites com pouca radiação da lua, e consequentemente seu ciclo de funcionamento é de aproximadamente $10 \%$ do tempo.

\subsection{Reconstrução dos eventos}

\subsubsection{Geometria}

\section{Detectores de superfície}

Para os eventos medidos apenas pelos detectores de superfície, é possível determinar o 
eixo do chuveiro (consequentemente a direção da partícula primária) através das diferenças de tempo entre os disparos dos tanques Cherenkov. O primeiro parâmetro calculado é uma estimativa da posição em que o eixo do chuveiro intercepta o plano dos detectores, o chamado baricentro. Este cálculo é feito através da média das distâncias dos tanques, ponderada pelos valores dos sinais em cada um. Sendo $\vec{x}_{i}$ e $S_{i}$ a distância e o sinal de um dado tanque respectivamente, a posição $\vec{x}_{0}$ do baricentro é calculada por,

$$
\vec{x}_{0}=\frac{\sum_{i} \sqrt{S_{i}} \vec{x}_{i}}{\sum_{i} \sqrt{S_{i}}} .
$$

De maneira análoga, sendo $t_{i}$ o tempo de detecção de um dado tanque, o tempo $t_{0} \mathrm{em}$ que a frente do chuveiro alcança a posição $\vec{x}_{0}$ é dada por,

$$
t_{0}=\frac{\sum_{i} \sqrt{S_{i}} t_{i}}{\sum_{i} \sqrt{S_{i}}} .
$$

Detalhes sobre estes cálculos estão na referência (62). O valor de $\vec{x}_{0}$ calculado pela equação 3.1 é utilizado como valor inicial para o ajuste da função de distribuição lateral, descrita na seção 3.3.2.

A próxima etapa na reconstrução da geometria do chuveiro é a determinação da direção do eixo e será descrita aqui a partir da suposição de frente esférica do chuveiro. O mesmo cálculo pode ser feito considerando frente plana ou frente parabólica. No caso da frente plana os resultados são muito menos precisos e para frente parabólica os resultados são muito próximos dos encontrados usando frente esférica. Sendo $R$ o valor do raio da esfera que aproxima a frente do chuveiro e $\hat{a}$ o versor que indica a direção do eixo, temos que o tempo em que a frente alcança a posição $\vec{x}$ é,

$$
t(\vec{x})=t_{0}+\frac{1}{c}|R \hat{a}-\vec{x}|,
$$

em que $c$ é a velocidade da luz e $t_{0}$ é o tempo em que a frente do chuveiro alcança a posição $\vec{x}_{0}$.

E portanto, sendo $\sigma\left(t_{i}\right)$ a incerteza em cada medida $t_{i}$, é possível determinar a direção do eixo do chuveiro $\hat{a}$ e o raio $R$ através da minimização de

$$
\chi^{2}=\sum_{i}\left(\frac{t_{i}-t_{0}-\frac{1}{c}\left|R \hat{a}-\vec{x}_{i}\right|}{\sigma\left(t_{i}\right)}\right)^{2} .
$$

Os detalhes da reconstrução geométrica a partir dos detectores de superfície podem ser encontrados na referência (63). A precisão na determinação da direção para os eventos medidos 
somente pelos tanques Cherenkov é da ordem de $1^{\circ}$ para eventos com energia da ordem de $10^{19} \mathrm{eV}(64)$.

\section{Telescópios de fluorescência}

A determinação da geometria dos chuveiros através dos telescópios de fluorescência é feita baseada nos tempos de disparo dos pixeis das câmeras. A caracterização geométrica do chuveiro é feita através do plano detector-chuveiro (SDP, em inglês) e dois parâmetros, $R_{p}$ e $\chi_{0}$, mostrados a figura 3.4. O SDP é o plano que contém o eixo do chuveiro e os vetores que ligam cada ponto medido do eixo ao seu respectivo pixel. $R_{p}$ é a distância perpendicular entre o detector e o eixo do chuveiro e $\chi_{0}$ é o ângulo entre o eixo do chuveiro e a linha horizontal contida no SDP. O tempo de disparo de um pixel que enxerga um ângulo $\chi$ é dado por (65):

$$
t(\chi)=t_{0}+\frac{R_{p}}{c} \operatorname{tg}\left[\left(\chi_{0}-\chi\right) / 2\right] .
$$

Usando os ângulos $\chi_{i}$ medidos em um tempo $t_{i}$, podemos encontrar $R_{p}$ e $\chi_{0}$ minimizando a função

$$
\chi^{2}=\sum_{i}\left(\frac{t_{i}-t_{0}-\frac{R_{p}}{c} \operatorname{tg}\left[\left(\chi_{0}-\chi_{i}\right) / 2\right]}{\sigma\left(t_{i}\right)}\right)^{2},
$$

em que $\sigma\left(t_{i}\right)$ é a incerteza experimental na medida de $t_{i}$. A figura 3.5 mostra um evento medido por um telescópio do Observatório Pierre Auger. A figura 3.5(a) mostra o campo de visão de cada pixel em elevação e azimute. Os pixel coloridos receberam um sinal e a escala de cores representa o tempo de disparo de cada pixel. A linha vermelha ajustada aos pontos medidos mostra a direção do SDP. A figura 3.5(b) mostra o tempo de disparo de cada pixel em função do ângulo $\chi$. A linha vermelha ajustada aos dados determina a direção do eixo do chuveiro. O ponto preto é o tempo de disparo de um tanque que foi usado para fazer a reconstrução híbrida (ver seção abaixo). Mais detalhes sobre a reconstrução da geometria dos eventos pelos telescópios de fluorescência podem ser encontrados nas referências (65, 66).

\section{Reconstrução híbrida}

A reconstrução híbrida da geometria é feita seguindo basicamente o mesmo procedimento da reconstrução através das medidas de fluorescência. A única diferença está na inclusão da chamada hottest station, que é a estação do detector de superfície com maior sinal. Os valores de $t$ e $\chi$ dessa estação são adicionados na equação 3.6. A adição desta estação é muito importante nos casos em que o traço medido pelo telescópio de fluorescência é muito curto, pois o ajuste da função da equação 3.6 não é bem definido. Isso significa que existem uma família de parâmetros $R_{p}$ e $\chi_{0}$ capazes de descrever os dados medidos apenas pelos pixeis das câmeras e portanto a incerteza na determinação da geometria do chuveiro é alta. A inclusão 


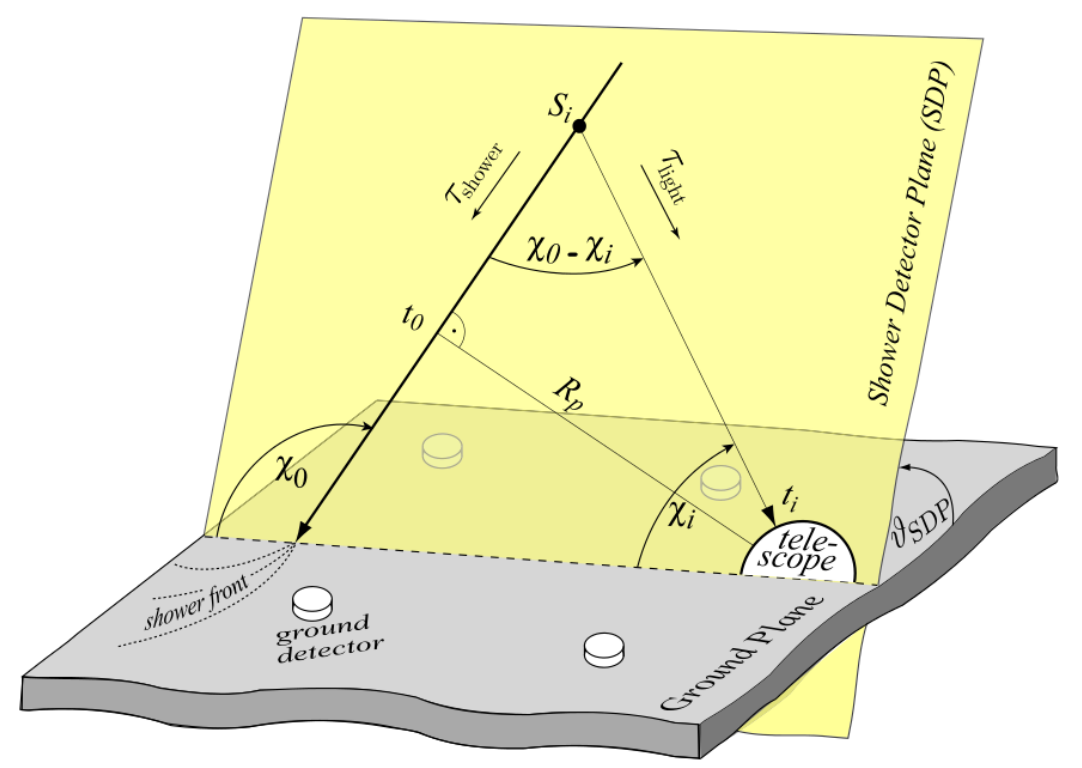

Figura 3.4 - Representação esquemática da geometria dos chuveiros medidos pelos telescópios de fluorescência, mostrando os parâmetros geométricos utilizados na reconstrução. Fonte: Imagem extraída da referência (65).

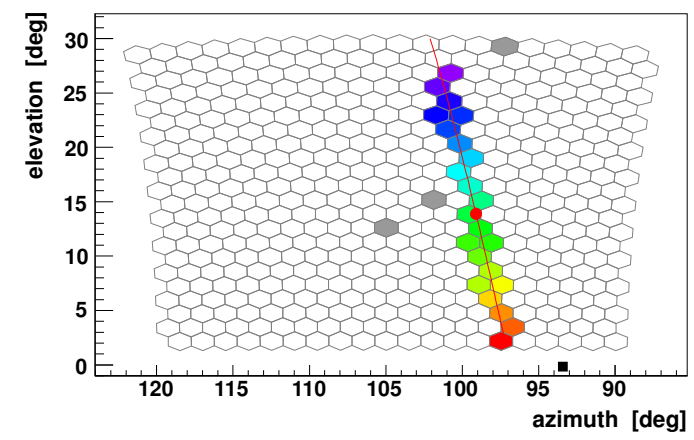

(a)

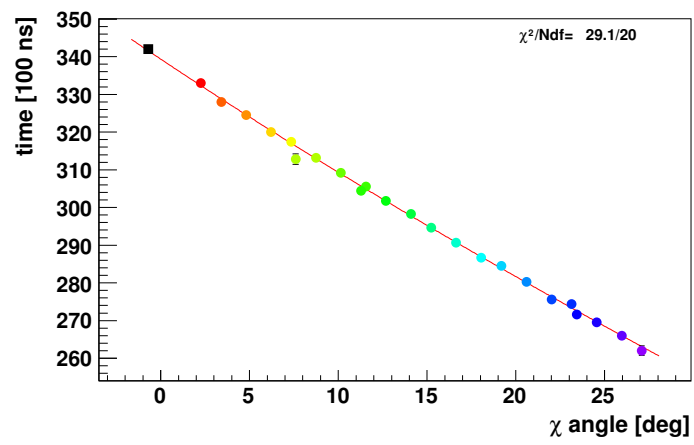

(b)

Figura 3.5 - (a) Gráfico mostrando os pixeis de um dos telescópios ao medir um chuveiro atmosférico. A escala de cores representa o tempo da medida. (b) Dados de $t \times \chi$ para um evento medido pelo telescópio ajustado pela função mostrada na equação 3.5 . O ponto preto representa o hottest station usada na reconstrução híbrida. Fonte: Imagens elaboradas pelo autor. 
dos valores medidos pela estação com maior sinal diminui drasticamente esta degenerescência, proporcionando maior precisão na reconstrução.

Como o ciclo de funcionamento dos detectores de superfície é de aproximadamente $100 \%$, quase todos os eventos medidos pelos telescópios de fluorescência são híbridos. Apesar de abaixo de $3 \times 10^{18} \mathrm{eV}$ a eficiência de trigger dos detectores de superfície não serem $100 \%$, a reconstrução híbrida não é prejudicada nesses casos, pois apenas uma estação é necessária. A precisão no valor da direção da partícula primária medida pelo procedimento acima é da ordem de $0.6^{\circ}$ (64). Mais detalhes sobre a reconstrução híbrida da geometria pode ser encontrada na referência (66).

\subsubsection{Função de distribuição lateral}

A partir da intensidade dos sinais dos detectores de superfície medidos em função do

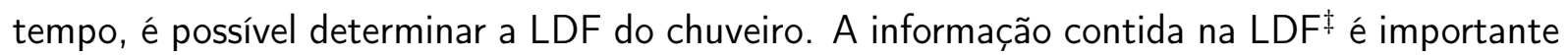
para determinar parâmetros geométricos do chuveiro, como posição do core (posição em que o eixo do chuveiro intercepta o plano dos detectores de superfície) e a direção, e também na determinação da energia através da calibração cruzada entre as medidas dos tanques e dos telescópios de fluorescência (ver seção 3.3.3).

A definição da função que descreve o sinal $S$ dos detectores de superfície em função da distância ao eixo do chuveiro $r$ é uma etapa importante no processo de reconstrução da LDF (67). Na referência (68) foi proposta uma função do tipo $\mathrm{NKG}^{\S}$, dada por,

$$
S(r)=k\left(\frac{r}{r_{s}}\right)^{-\beta}\left(1+\frac{r}{r_{s}}\right)^{-\beta},
$$

em que $\beta$ é uma função do ângulo zenital do chuveiro $(\beta=\beta(\theta))$, e $r_{s}$ é um parâmetro constante que representa o fator de escala dos chuveiros. A partir da função proposta, foi mostrado na referência (68) que o valor de $r$ no qual as flutuações devido a incerteza na forma da LDF são menos significativas é de aproximadamente $1000 \mathrm{~m}$ para um arranjo de superfície com as configurações do Observatório Pierre Auger. Por esse motivo, o valor de $S_{1000}$ é utilizado para determinar a energia da partícula primária.

A função utilizada pelo Observatório Pierre Auger atualmente é uma derivação da função

\footnotetext{
$\ddagger$ Lateral Distribution Function
}

§NKG são as iniciais de Nishimura \& Kamata (69) e Greisen (70), que propuseram essa classe de funções. 


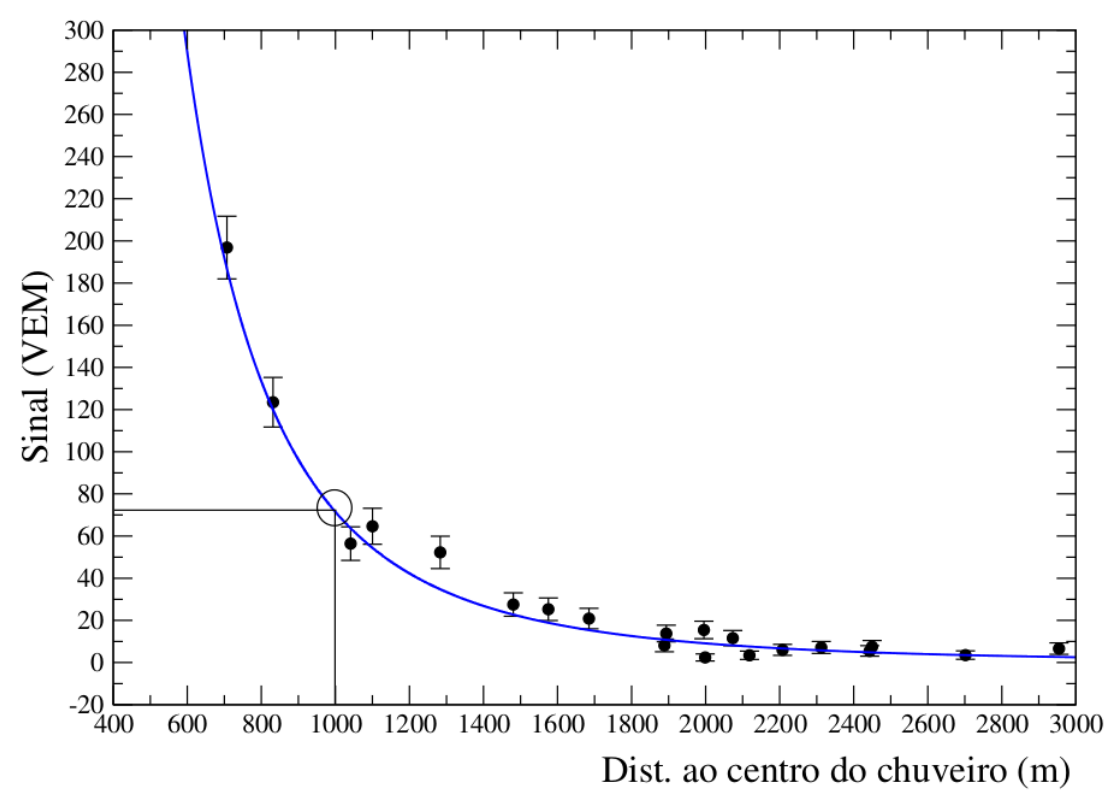

Figura 3.6 - Função de distribuição lateral de um evento medido pelo Observatório Pierre Auger. Os pontos pretos mostram os sinais medidos por cada estação e a curva azul é uma função dada pela equação 3.8 ajustada aos dados. Fonte: Imagem extraída da referência (71).

mostrada na equação 3.7 e é dada por,

$$
S(r)=S_{1000}\left(\frac{r}{1000}\right)^{-\beta}\left(\frac{r+700}{1700}\right)^{-\beta} .
$$

A dependência com a energia e com o ângulo zenital da forma da função está incorporado no parâmetro $\beta$. A figura 3.6 mostra um exemplo de uma LDF medida pelo Observatório Pierre Auger. A parametrização de $\beta(E, \theta)$ é feita através de simulações de Monte Carlo, como mostrado nas referências $(71,72)$.

Devido a fatores geométricos dos chuveiros, o parâmetro $S_{1000}$ obtido através do ajuste da função dada pelo equação 3.8 é fortemente dependente do ângulo zenital $\theta$. Isso pode ser observado na figura 3.7 que mostra a chamada curva de atenuação, ou seja, $S_{1000}$ em função de $\cos ^{2}(\theta)$. A função definida para descrever essa relação é $S_{1000}(\theta)=1+a x+b x^{2}$, em que $x=\cos ^{2}(\theta)-\cos ^{2}(\langle\theta\rangle)$. Nesse caso, $\langle\theta\rangle=38^{\circ}$. Essa curva de atenuação será importante para a calibração da energia, como será mostrado na seção 3.3.3

\subsubsection{Perfil longitudinal e energia}

Após determinar a geometria dos chuveiros, é possível reconstruir o perfil longitudinal e consequentemente a energia da partícula primária através dos dados medidos pelos telescópios 


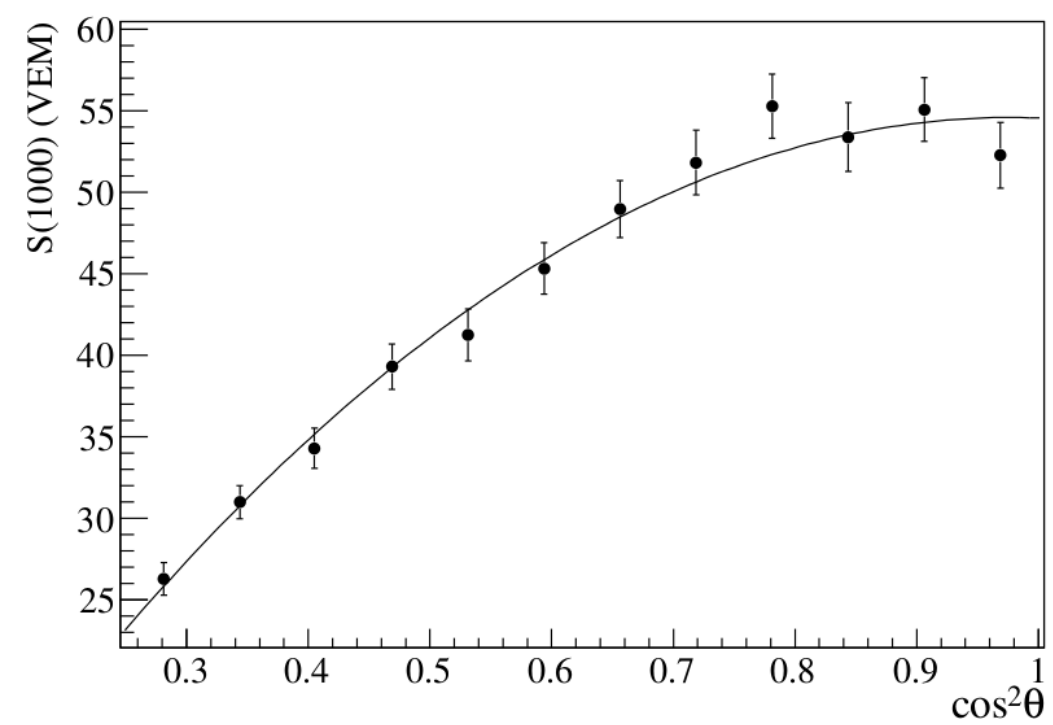

Figura 3.7 - Curva de atenuação utilizada para obter o parâmetro $S_{38}$. Os pontos mostram a média do sinal medido em um intervalo de ângulo zenital. Em cada intervalo, estão incluídos todos os eventos medidos pelo Observatório Pierre Auger que sobreviveram aos cortes de qualidade desta análise. Fonte: Imagem extraída da referência (73).

de fluorescência. A energia depositada pelo chuveiro na atmosfera em função da profundidade pode ser obtida através da conversão da radiação medida em cada pixel da câmera (74). Para obter a função que descreve o perfil longitudinal $\frac{d E}{d X}(X)$, ajusta-se uma função do tipo Gaisser-Hillas (75), dada por,

$$
\frac{d E}{d X}(X)=\frac{d E}{d X}_{\max } \cdot\left(\frac{X-X_{0}}{X_{\max }-X_{0}}\right)^{\frac{X_{\max }-X_{0}}{\lambda}} \cdot \exp \left(\frac{X_{\max }-X}{\lambda}\right)
$$

em que $\frac{d E}{d X}{ }_{\max }, X_{\max }, X_{0}$ e $\lambda$ são parâmetros do ajuste. A energia calorimétrica é calculada através da integral do perfil longitudinal ajustado por 3.9.

$$
E_{c a l}=\int \frac{d E}{d X} d X
$$

A figura 3.8 mostra um exemplo de um perfil longitudinal medido pelo Observatório Pierre Auger. Os pontos pretos são as intensidades do sinal medido para dada profundidade atmosférica. A linha vermelha é a função Gaisser-Hillas ajustada nesse evento e o ponto vermelho indica a posição do $X_{\max }$.

Para obter a energia total do chuveiro $E_{F D}$ a partir da energia calorimétrica $E_{c a l}$ aplica-se uma correção para compensar a chamada "energia invisível" (76), que é a energia carregada por partículas que não são detectadas pelos telescópios, como múons e neutrinos.

No caso de eventos que não são medidos pelos telescópios de fluorecência, mas somente pelos detectores de superfície, a energia total do chuveiro é determinada através da calibração 


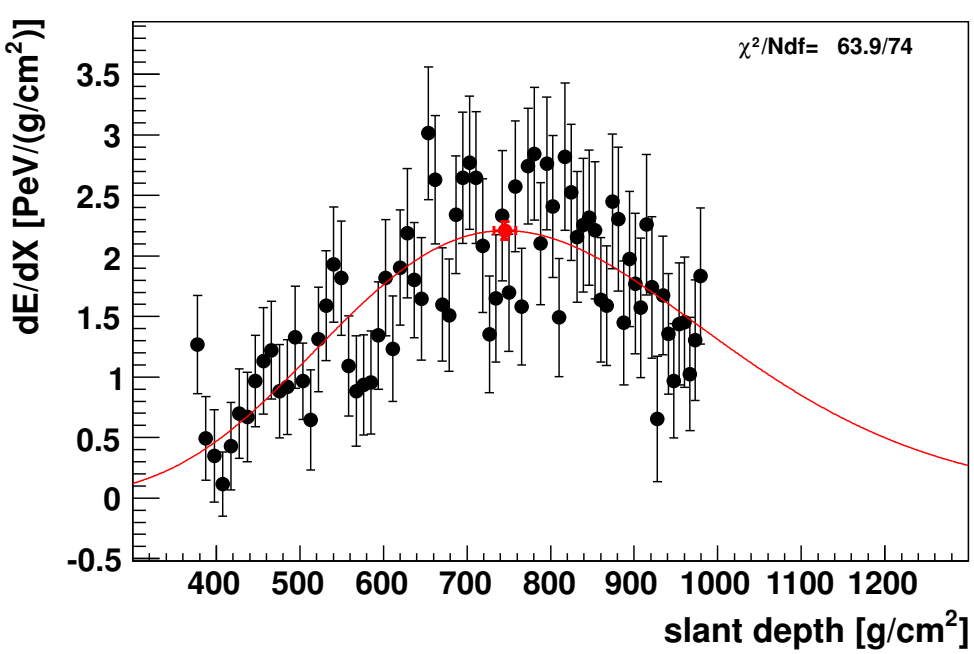

Figura 3.8 - Exemplo de um perfil longitudinal medido pelos telescópios de fluorescência. Os pontos pretos são os sinais medidos pelos telescópios em cada intervalo de profundidade atmosférica. O ponto vermelho é o valor de $X_{\max }$ ajustado. A curva vermelha é a função Gaisser-Hillas ajustada aos dados. Fonte: Imagem elaborada pelo autor.

cruzada, utilizando um parâmetro chamado $S_{38}$ e a energia $E_{F D}$ calculada através do perfil longitudinal (73). $S_{38}$ é definido por $S_{38}=S_{1000} / S_{1000}(\theta)$ e é um parâmetro independente do ângulo zenital. A figura 3.9 mostra um gráfico com a correlação entre $S_{38}$ e $E_{F D}$. A linha mostra o ajuste linear utilizado como base para a calibração. 


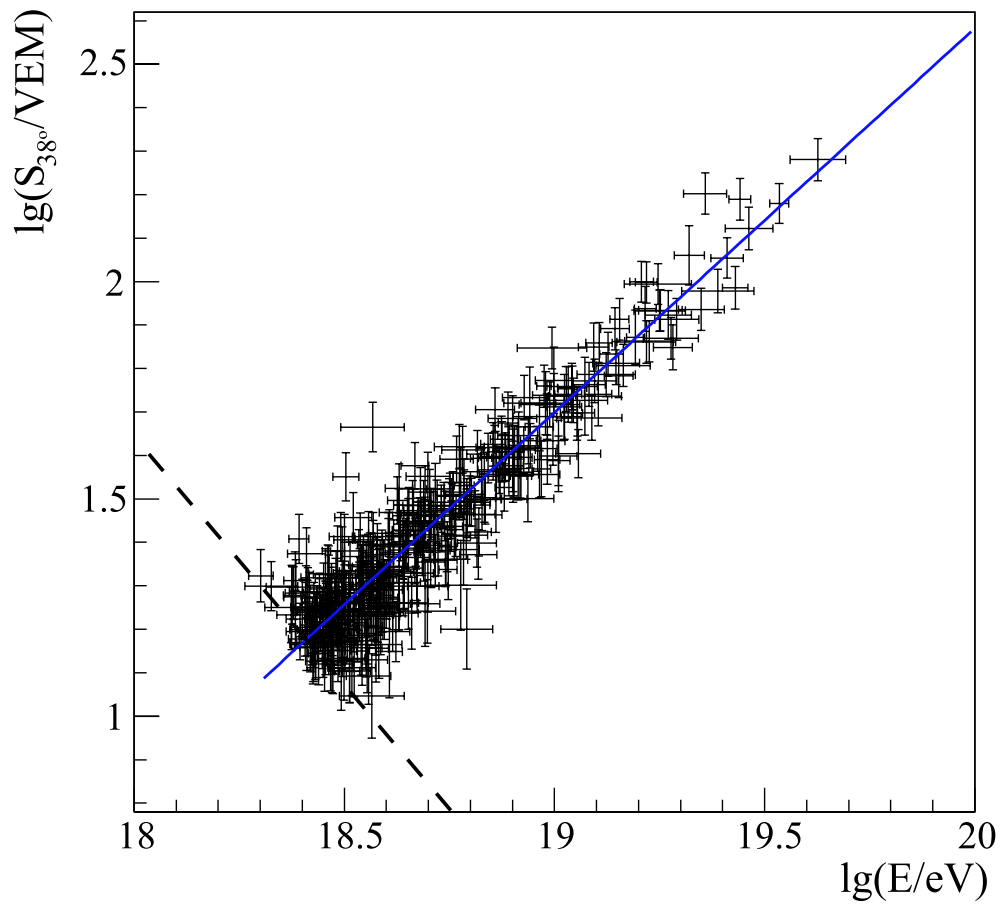

Figura 3.9 - Relação entre $S_{38}$ e $E_{F D}$ para um subconjunto de eventos medidos pelo Observatório Pierre Auger. A linha cinza é a função utilizada para obter a energia da partícula primária a partir do parâmetro $S_{38}$, para os eventos medidos apenas pelos detectores de superfície. Fonte: Imagem extraída da referência (77). 


\section{Taxa de elongação: métodos de deconvolução}

\subsection{Introdução}

\subsubsection{O problema da deconvolução}

Em experimentos de física de altas energias, as distribuições de grandezas medidas são comumente distorcidas por efeitos dos detectores, enviesando os resultados e impedindo a comparação direta destes com modelos teóricos ou com resultados de outros experimentos. As principais causas destas distorções são: resolução finita, eficiência limitada e transformação. Resolução finita significa que o detector não é capaz de medir as grandezas físicas com precisão absoluta e portanto existe uma incerteza natural inerente aos valores medidos. Os efeitos da eficiência limitada têm origem no fato de que a probabilidade do detector medir certos eventos é menor que 1 e pode variar com as características de cada evento. Por fim, a transformação se refere a tendência inerente aos detectores de alterar de maneira sistemática os valores reais das grandezas de acordo com características específicas dos instrumentos aplicados no processo de medida.

Esse problema tem sido extensivamente abordado ao longo das últimas décadas, o que resultou no desenvolvimento de uma série de métodos estatísticos que têm como objetivo extrair o efeito dos detectores das distribuições medidas gerando resultados sem vieses experimentais. Embora problemas muito semelhantes a este apareçam em outros contextos, aqui neste trabalho iremos nos focar em contextualizar a questão do ponto de vista dos experimentos de física de altas energias.

Em um formalismo matemático, o efeito dos detectores pode ser representado por uma função de duas variáveis, chamada função resposta do detector, que relaciona o valor da grandeza física medida $(y)$ com seu valor real $(x)$. Neste sentido, as funções das grandezas medidas são produtos da convolução da função da grandeza real com a função resposta do 
detector. Podemos então representar o problema formalmente como uma equação integral do tipo,

$$
G(y)=\int A(y, x) F(x) d x,
$$

em que $G(y)$ é a função da variável medida, $F(x)$ é a função da variável real e $A(y, x)$ é a função resposta do detector. A equação diferencial acima é chamada de equação de Fredholm de primeiro tipo e é um exemplo de um problema de inversão (78).

$\mathrm{Na}$ grande maioria das situações reais, o problema deve ser tratado de maneira discreta, e portanto a equação integral acima se torna um sistema linear de equações que escrevemos na forma matricial como,

$$
\vec{y}=\hat{A} \cdot \vec{x},
$$

em que $\vec{y}$ é $\circ$ vetor que representa a distribuição medida, $\vec{x}$ é o vetor que representa a distribuição real e $\hat{A}$ é a função resposta do detector em sua forma matricial, também chamada de matriz resposta do detector. No contexto dos métodos de deconvolução, a matriz resposta pode ser interpretada como uma matriz de probabilidade, na qual cada entrada $\hat{A}_{i j}$ representa a probabilidade de medirmos uma grandeza física com valor $y_{j}$ sendo que o valor real é $x_{i}$.

Em física de altas energias, geralmente é possível se obter a matriz de resposta através de simulações de Monte Carlo. Embora esse fato seja essencial para a abordagem deste problema, a dependência com modelos teóricos-computacionais pode gerar consequências negativas. $O$ principal exemplo são os erros sistemáticos na construção da matriz devido a incertezas nos modelos teóricos utilizados pelas simulações.

Devido às características da matriz resposta, a solução direta do sistema mostrado na equação 4.2 , invertendo a matriz resposta $\left(\vec{x}=\hat{A}^{-1} \vec{y}\right)$, tende a amplificar flutuações estatísticas inerentes à distribuição medida e gerar uma solução que sofre com muitas oscilações e não têm portanto significado físico. Por esse motivo, o problema é chamado de "malposto" e sua abordagem exige métodos estatísticos específicos que, através de aproximações, fornecem soluções adequadas para um contexto físico. Tais métodos são chamados métodos de deconvolução* e são usualmente divididos em duas classes: métodos de regularização e métodos iterativos. Nesse trabalho foram estudados e implementados dois métodos, sendo um de cada classe. O primeiro é chamado método $S V D^{\dagger}(4)$, pertence à classe dos métodos de regularização e será descrito na seção 4.1.2.1. O segundo é chamado método iterativo bayesiano (5), pertence a classe dos métodos iterativos e será descrito na seção 4.1.2.2.

\footnotetext{
*Em inglês esses métodos são chamados de unfolding methods. Neste trabalho escolhemos livremente traduzir o nome para métodos de deconvolução. Outra tradução possível seria métodos de desdobramento.

†SVD é a sigla em inglês para Singular Values Decomposition.
} 
Os métodos de deconvolução tem sido extensivamente utilizados no contexto de física de altas energias (79-81). Algumas das primeiras aplicações foram feitas pela colaboração CHARM (82, 83) na década de 80. Recentemente podemos destacar algumas aplicações pelos experimentos do CERN associados ao LHC(Large Hadron Collider), como o ATLAS (84), o ALICE (85) e o CMS (86). Em astrofísica de partículas, métodos de deconvolução têm sido utilizados pela colaboração do KASCADE em uma forma bidimensional para obter espectros de energias de diferentes grupos de partículas primárias (87-89).

No presente trabalho, os métodos de deconvolução serão aplicados às distribuições de $X_{\max }$ medidas pelo Observatório Pierre Auger. A partir das distribuições deconvoluídas será possível avaliar a taxa de elongação dos dados medidos, compará-la com modelos teóricos e assim obter informações sobre a composição média dos raios cósmicos (ver seção 2.4).

A análise padrão que tem sido realizada pela colaboração do Observatório Pierre Auger (90), sem a utilização de métodos de deconvolução, corrige os efeitos do detector através da eliminação de eventos que possam gerar vieses nas medidas. O filtro de eventos é aplicado em duas etapas. Primeiramente, os efeitos da eficiência limitada e da transformação são corrigidos através de cortes nos eventos, os chamados cortes de fidelidade (3). Através da análise dos dados, são definidas regiões geométricas, considerando a posição do chuveiro com relação ao campo de visão do telescópio de fluorescência, na quais o efeito do detector é em média nulo, e então são selecionados apenas eventos que estejam dentro dessa região. Os cortes de fidelidade são bastante eficientes em garantir um espaço paramétrico de medidas no qual o detector não produz viés na determinação da média e do $R M S$ da distribuição de $X_{\max }$. No entanto, isto é conseguido através do descarte de muitos eventos, o que diminui o poder estatístico da análise.

A segunda etapa da análise calcula o efeito de resolução finita utilizando simulações de Monte Carlo. O resultado desta análise mostra que o descolamento do $\left\langle X_{\max }\right\rangle$ é em média nulo enquanto que o $R M S$ é diferente de zero. Os métodos de deconvolução buscam eliminar os mesmos três efeitos (resolução finita, eficiência limitada e transformação) minimizando o descarte de eventos de forma a maximizar o poder estatístico da análise. 


\subsubsection{Algoritmos de deconvolução}

\subsubsection{Método SVD}

O método SVD implementado neste trabalho pertence a classe dos algoritmos de regularização e foi baseado no trabalho de Hocker e Kartvelishvili (4). Os princípios básicos dessa classe de métodos já havia sido desenvolvido por Blobel (79), e foi posteriormente adaptado alcançando o estado que será descrito aqui. A ideia geral do algoritmo é transformar o sistema inicial do problema de deconvolução (equação 4.2) em um sistema diagonal a partir da decomposição em valores singulares (SVD), e então resolver esse sistema através de um processo de regularização. Algumas etapas intermediárias são necessárias, como o redimensionamento das equações e a normalização das variáveis. A seguir descreveremos resumidamente cada etapa do algoritmo considerando o formalismo e algumas especificidades do problema abordado neste trabalho.

\section{Decomposição em valores singulares}

A decomposição em valores singulares de uma matriz $\hat{A}$, quadrada de ordem $n^{\ddagger}$, é definida da seguinte maneira,

$$
\hat{A}=U S V^{T}
$$

em que $U$ e $V$ são matrizes ortogonais quadradas de ordem $n$ e $S$ é uma matriz diagonal com elementos não negativos. Os elementos da diagonal de $S$ são chamados valores singulares $\left(S_{i i}=s_{i}\right)$ e as colunas das matrizes $U$ e $V$ são chamadas vetores singulares. Os valores singulares são comumente indexados em ordem crescente, sendo $s_{1} \circ$ maior e $s_{n}$ o menor deles.

Utilizando a decomposição da matriz $\hat{A}$ mostrada na equação 4.3, o sistema mostrado na equação 4.2 pode ser escrito como $\vec{y}=U S V^{T} \vec{y}$. A partir desse novo sistema, uma vez que as matrizes $U$ e $V$ são ortogonais $\left(U^{-1}=U^{T}\right.$ e $\left.V^{-1}=V^{T}\right)$, podemos multiplicar os dois termos por $U^{T}$ pela esquerda e obter $U^{T} \vec{y}=S V^{T} \vec{x}$. Uma mudança de variáveis do tipo $\vec{d}=U^{T} \vec{y}$ e $\vec{z}=V^{T} \vec{x}$ permite agora escrever o problema na forma,

$$
\vec{d}=S \vec{z}
$$

que é um sistema linear diagonal, com $n$ equações desacopladas. A solução do sistema mostrado na equação 4.4 é dada simplesmente por $z_{i}=d_{i} / s_{i}$, em que $z_{i}$ e $d_{i}$ são componentes

\footnotetext{
${ }^{\ddagger}$ A decomposição em valores singulares pode ser aplicada a matrizes não quadradas.
} 
dos vetores $\vec{d}$ e $\vec{z}$. A partir dessa solução é possível realizar a mudança de variáveis inversa e obter a solução do sistema inicial, dada por $\vec{x}=V \vec{z}$.

\section{Normalização das variáveis e redimensionamento das equações}

Mudanças de variáveis são comumente utilizadas na solução de sistemas de equações com o objetivo de facilitar a solução. Entre as muitas maneiras possíveis de realizar a mudança de variáveis, deve ser escolhida aquela que ameniza características indesejáveis do problema inicial de modo que o comportamento do novo sistema não seja dominado por essas características. No presente algoritmo, a escolha foi transformar as variáveis de modo que as novas variáveis sejam $w_{i}=x_{i} / x_{i}^{i n i}$ e os elementos da nova matriz sejam $A_{i j}=\hat{A}_{i j} x_{i}^{i n i}$, em que $\vec{x}^{i n i}$ é uma distribuição esperada para a solução e portanto contém informações a priori sobre como deve ser $\vec{x}$.

Em muitas aplicações, a distribuição $\vec{x}^{i n i}$ é gerada a partir de simulações de Monte Carlo. Entretanto, no caso do problema abordado neste trabalho, as distribuições geradas por Monte Carlo devem necessariamente conter informações sobre a composição das partículas primárias e sobre o modelo de interação hadrônica. Isso significa que as suposições sobre composição e as incertezas inerentes aos modelos de interação hadrônica afetariam sistematicamente a forma da distribuição gerada. Por esse motivo, decidimos utilizar como distribuição $\vec{x}^{i n i}$ a distribuição medida $\vec{y}$, pois apesar de ser essencialmente diferente da distribuição real $\vec{x}$, ainda é mais semelhante a esta que as distribuições geradas por simulações de Monte Carlo. O novo sistema a ser resolvido após a mudança de variáveis é,

$$
A \cdot \vec{w}=\vec{y}
$$

e a solução do sistema dado pela equação 4.2 pode ser obtida através da solução do sistema mostrado na equação 4.5 da seguinte maneira,

$$
x_{i}=w_{i} x_{i}^{i n i}, \quad i=1, \ldots, n .
$$

A próxima etapa é o redimensionamento das equações e tem como objetivo balancear as equações de acordo com a incerteza experimental em cada bin da distribuição medida. A maneira como isso é realizado na referência (4) considera um caso geral em que a matriz de covariância da distribuição medida pode não ser diagonal, em outras palavras, pode haver correlação entre os eventos medidos.

No contexto deste trabalho, o procedimento torna-se mais simples, uma vez que os eventos medidos pelo Observatório Pierre Auger são independentes entre si e portanto a matriz de covariância $Y$ é diagonal $\left(Y_{i j}=Y_{i}\right.$ se $i=j$ e $Y_{i j}=0$ se $\left.i \neq j\right)$, e os valores da diagonal 
representam as variância estatística em cada bin, logo, $Y_{i}=\sigma_{i}^{2}$. Como os valores de cada bin são contagens de eventos e seguem distribuições de Poisson, a variância será $\sigma_{i}=\sqrt{y_{i}}$ e portanto $Y_{i}=y_{i}$. Nesse caso o redimensionamento gera as seguintes equações,

$$
\tilde{A}_{i j}=A_{i j} / \sqrt{y_{i}}
$$

e

$$
\tilde{y}_{i}=y_{i} / \sqrt{y_{i}}=\sqrt{y_{i}}
$$

O sistema a ser resolvido agora é,

$$
\tilde{A} \vec{w}=\tilde{y} \Rightarrow \sum_{j} \tilde{A}_{i j} w_{i}=\tilde{y}_{j}
$$

\section{Regularização e desdobramento}

O sistema dado pela equação 4.9 pode ser resolvido pelo método dos mínimos quadrados através da minimização de,

$$
\chi^{2}=(\tilde{A} \vec{w}-\tilde{y})^{T}(\tilde{A} \vec{w}-\tilde{y}) .
$$

A solução da equação 4.10, apesar das mudanças de variáveis e redimensionamento das equações, ainda sofre pelas instabilidades inerentes ao problema da deconvolução. Esse problema é resolvido pela adição de um termo de regularização (91), que transforma a equação 4.10 em,

$$
\chi^{2}=(\tilde{A} \vec{w}-\tilde{y})^{T}(\tilde{A} \vec{w}-\tilde{y})+\tau \cdot(C \cdot \vec{w})^{T}(C \cdot \vec{w}),
$$

em que $C$ é a matriz que define a condição a priori que a solução deve seguir e $\tau$ é o peso relativo que essa condição terá na solução do sistema. No caso do algoritmo apresentado aqui, a solução desejada deve ser suave, sem grandes oscilações entre os bins. Portanto, a matriz $C$ utilizada deve representar a "suavidade", que pode ser medida através da curvatura da solução. A matriz $C$ que define a curvatura da solução é,

$$
C=\left(\begin{array}{cccccc}
-1 & 1 & 0 & 0 & \ldots & \\
1 & -2 & 1 & 0 & \ldots & \\
0 & 1 & -2 & 1 & \ldots & \\
& \ldots & & & \ldots & \\
& \cdots & & 1 & -2 & 1 \\
& \ldots & & & 1 & -1
\end{array}\right)
$$


A minimização de $\chi^{2}$ na equação 4.11 implica na solução de um novo sistema linear,

$$
\left[\begin{array}{c}
\tilde{A} \\
\sqrt{\tau} \cdot C
\end{array}\right] \vec{w}=\left[\begin{array}{l}
\tilde{y} \\
0
\end{array}\right]
$$

É possível alcançar a solução deste sistema através da decomposição em valores singulares, porém este processo deveria ser executado para cada valor de $\tau$ separadamente. Para superar essa dificuldade, uma solução alternativa foi proposta nas referências (4, 91), na qual a solução para qualquer valor de $\tau$ é obtida através da solução para $\tau=0$. Os detalhes desse procedimento podem ser encontrados na referência (4). Apresentaremos apenas as etapas mais relevantes para a compreensão do algoritmo.

A primeira etapa é tornar o termo de regularização proporcional à matriz identidade, transformando o sistema dado pela equação 4.13 , em

$$
\left[\begin{array}{c}
\tilde{A} \cdot C^{-1} \\
\sqrt{\tau} \cdot I
\end{array}\right] C \vec{w}=\left[\begin{array}{c}
\tilde{y} \\
0
\end{array}\right]
$$

Utilizando a decomposição em valores singulares para resolver o sistema mostrado na equação 4.14 para $\tau=0$, temos a seguinte decomposição,

$$
\tilde{A} C^{-1}=U S V^{T}
$$

em que as matrizes $U, V$ e $S$ seguem a descrição já apresentada neste texto. Usando as matrizes $U$ e $V$ (e suas respectivas transpostas) para rotacionar o sistema e obter um sistema diagonal, temos os novos vetores $\vec{d}=U^{T} \tilde{b}$ e $\vec{z}=V^{T} C \vec{w}$. O sistema diagonal agora é o seguinte,

$$
S \cdot \vec{z}=\vec{d}
$$

levando a solução, $z_{i}^{\tau=0}=\frac{d_{i}}{s_{i}}$ e $w^{\tau=0}=C^{-1} V z^{\tau=0}$. A partir dessa etapa a solução para $\tau \neq 0$ pode ser obtida (como descrito em detalhes na referência (91)), levando a solução,

$$
d_{i}^{\tau}=d_{i} \frac{s_{i}^{2}}{s_{i}^{2}+\tau},
$$

e portanto,

$$
z_{i}^{\tau}=\frac{d_{i} s_{i}}{s_{i}^{2}+\tau}, \quad w^{\tau}=C^{-1} V z^{\tau}
$$

A partir da equação 4.18 pode-se observar o efeito do termo de regularização $\tau$ na solução final. Para as equações com valores altos de $s_{i}$, o efeito da regularização é insignificante, porém, se os valores de $s_{i}$ são baixos para dada equação, o fator de regularização atua amenizando 
o peso desta na solução final e portanto eliminando o problema das rápidas oscilações nestas equações.

A última etapa do algoritmo é a escolha do fator de regularização $\tau$ e isso é feito avaliando a informação contida no gráfico de $\vec{d} \times i$. Os valores de $d_{i}$ são as componentes do vetor medido y na nova base, após a decomposição. Cada $d_{i}$ é uma medida do peso que dada equação terá na solução final. O gráfico de $\vec{d} \times i$ mostra dois comportamentos distintos. Os índices mais baixos tem os maiores valores de $d_{i}$ e representam as equações mais importantes para a solução e mais estáveis. O valor de $d_{i}$ tende a cair rapidamente até o comportamento se alterar para valores que oscilam próximos de zero. As equações definidas por este regime de $d_{i}$ são as equações que geram as instabilidades na solução e portanto o peso destas deve ser diminuído. Primeiramente, deve ser determinado o valor de $i$ que acontece essa transição. Em geral essa não é uma tarefa difícil, visto que poucas equações costumam ser importantes e ter valores significativos de $d_{i}$. Uma vez selecionado este valor, o $\tau$ é definido como $\tau=s_{k}^{2}$, sendo $k$ o índice da transição.

\subsubsection{Método iterativo bayesiano}

O segundo método implementado neste trabalho é baseado na referência (5) e será chamado aqui de método iterativo bayesiano ou somente método bayesiano. Métodos bayesianos usualmente são dependentes do conhecimento a priori sobre a forma da solução esperada. No algoritmo apresentado aqui, essa limitação é superada pelo processo iterativo aplicado, que ameniza a dependência do resultado com a solução esperada. Sendo novamente $x$ a variável real que queremos alcançar a distribuição e $y$ a variável medida pelo nosso detector, o teorema de Bayes pode ser escrito como,

$$
P\left(x_{i} \mid y_{j}\right)=\frac{P\left(y_{j} \mid x_{i}\right) P\left(x_{i}\right)}{P\left(y_{i}\right)}=\frac{P\left(y_{j} \mid x_{i}\right) P\left(x_{i}\right)}{\sum_{l} P\left(y_{j} \mid x_{l}\right) P\left(x_{l}\right)},
$$

em que $P\left(x_{i} \mid y_{j}\right)$ é a probabilidade condicional da variável real estar no bin $i$ tendo sido medido a variável $y_{j}$ no bin $j, P\left(y_{j} \mid x_{i}\right)$ é a probabilidade condicional de medir a variável $y_{j}$ no bin $j$ tendo que o valor real da variável é $x_{i}$ no bin $i$ e $P\left(x_{i}\right)$ é a distribuição de probabilidade da variável real. A partir dessas definições podemos associar $P\left(y_{j} \mid x_{i}\right)$ à matriz de resposta do detector $\left(P\left(y_{j} \mid x_{i}\right) \equiv \hat{A}_{i j}\right)$. Além disso, por definição, temos que,

$$
P\left(x_{i}\right)=\sum_{j} P\left(x_{i} \mid y_{j}\right) P\left(y_{j}\right)=\sum_{j} \frac{P\left(y_{j} \mid x_{i}\right) P\left(x_{i}\right)}{\sum_{l} P\left(y_{j} \mid x_{l}\right) P\left(x_{l}\right)} P\left(y_{j}\right) .
$$


A distribuição de probabilidade da variável medida pode ser identificada com a distribuição medida pelo detector $\left(P\left(y_{j}\right) \equiv y_{j}\right)$, a menos de um fator de normalização. Pela equação 4.20 e tendo conhecimento a priori da distribuição da variável real, é possível calcular a solução do problema $P\left(x_{i}\right)$. Entretanto, como já foi dito, será aplicado nessa etapa um processo iterativo para amenizar o efeito da distribuição real $P\left(x_{l}\right)$. Sendo $k$ o índice da iteração, temos que,

$$
P\left(x_{i}^{k+1}\right)=\sum_{j} \frac{P\left(y_{j} \mid x_{i}\right) P\left(x_{i}^{k}\right)}{\sum_{l} P\left(y_{j} \mid x_{l}\right) P\left(x_{l}^{k}\right)} P\left(y_{j}\right),
$$

em que $P\left(x_{i}^{0}\right)$ é a distribuição real inicial, que pode ser obtida através de simulações de Monte Carlo, porém se necessário também pode ser utilizada uma distribuição uniforme. A distribuição $P\left(x_{i}^{1}\right)$ obtida utilizando $P\left(x_{i}^{0}\right)$ será utilizada como distribuição inicial na segunda iteração para calcular $P\left(x_{i}^{2}\right)$. Esse processo se repete até que a solução $P\left(x_{i}\right)$ convirja. $\mathrm{O}$ critério de convergência pode ser definido através da comparação entre a solução para um dado valor de $k$ e a solução anterior de $k-1$. Na seção 4.4.4 descrevemos o critério escolhido para este trabalho.

\subsection{Construção da matriz resposta}

Como foi dito na seção 4.1.1, a matriz resposta do detector é um elemento muito importante na aplicação de métodos de deconvolução. No contexto deste trabalho, a matriz resposta do detector relaciona os valores de $X_{\max }$ medidos pelo Observatório Pierre Auger e os valores de $X_{\max }$ verdadeiros de cada evento. Através de simulações de Monte Carlo dos chuveiros e de todo o processo de medida do detector, é possível obter o valor de $X_{\max }$ medido para cada chuveiro com o valor de $X_{\max }$ verdadeiro conhecido. Após efetuar esse processo para um número suficiente de chuveiros, a matriz resposta é construída preenchendo um histograma bidimensional no qual o eixo $x$ contém os bins de $X_{\max }$ verdadeiro e o eixo $y$ contém os bins de $X_{\max }$ medido. Como os algoritmos de deconvolução utilizados nesse trabalho tratam a matriz resposta como a matriz de probabilidades, cada bin do eixo $x$ deve ser normalizado de modo a garantir que a soma dos eventos para cada um seja igual a 1 .

É de extrema importância para a eficiência dos métodos de deconvolução que a matriz resposta seja suave, sem grandes flutuações estatísticas entre os bins. Isso implica que as simulações utilizadas contenham um número suficientemente grande de chuveiros em todas as regiões de $X_{\max }$. Através dos softwares de simulação de chuveiros tradicionais, como CORSIKA (92) e CONEX (30, 31), esta condição é difícil de ser satisfeita, pois a distribuição de 


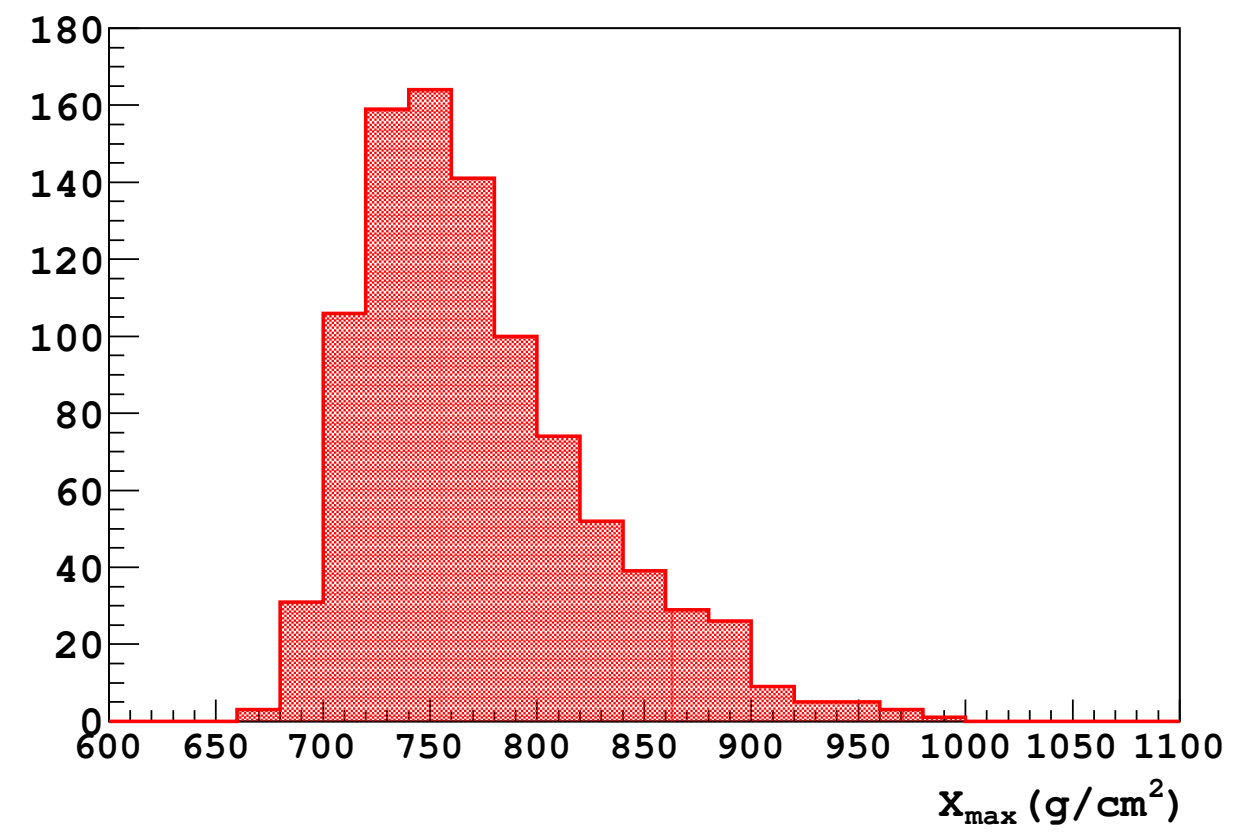

Figura 4.1 - Distribuição de $X_{\max }$ de chuveiros iniciados por prótons com energia de $10^{18.6} \mathrm{eV}$, gerados pelo CONEX. Fonte: Imagem gerada pelo autor.

$X_{\max }$ resultante contém um número muito baixo de chuveiros com valores consideravelmente maiores ou menores que o valor médio.

A figura 4.1 ajuda a ilustrar esse problema. A distribuição mostrada foi gerada a partir do software CONEX, considerando como partícula primária prótons com um valor fixo de energia de $10^{18.6} \mathrm{eV}$. O modelo de interação hadrônica utilizado foi Sibyll2.1 (93). É possível notar claramente que para $X_{\max } \sim 1000 \mathrm{~g} / \mathrm{cm}^{2}$ o número de chuveiros é muito baixo. Nesse caso, para construir a matriz resposta utilizando esse conjunto de simulações, seriam necessários simular um número extremamente elevado de chuveiros para obtermos uma boa amostragem nessa região e como consequência o custo computacional deste procedimento seria inviável.

Com o objetivo de solucionar o problema da subamostragem em algumas regiões de $X_{\max }$, foi implementado um sistema alternativo para simular o processo de medida do Observatório Pierre Auger. A ideia principal desse método é parametrizar a função Gaisser-Hillas (ver seção 3.3.3), que descreve os perfis longitudinais, de forma que seja possível simular a reconstrução de chuveiros atmosféricos para chuveiros com qualquer valor de $X_{\max }$. O procedimento implementado aqui foi baseado no algoritmo proposto na referência (94), que aplica a mesma ideia para parametrizar todos os parâmetros da função Gaisser-Hillas em função da energia e do tipo de partícula (somente prótons e núcleos de ferro).

O conjunto de chuveiros utilizado para parametrizar o perfil longitudinal foi gerado uti- 
lizando o software CONEX (30, 31) e o modelo de interação hadrônica utilizado foi o Sybill2.1 (93). Foram gerados chuveiros com energia entre $10^{17.0}$ e $10^{20.0} \mathrm{eV}$, em intervalos de $\Delta \log (E / e V)=0.1$. Os valores de massa nuclear dos primários foram $A=1,5,10,15,20,25,30,35,40,4$ Para cada valor de energia e massa do primário foram gerados 1000 chuveiros.

Após ajustar a função Gaisser-Hillas aos perfis longitudinais das simulações citadas acima, a próxima etapa do processo foi dividir os parâmetros $\log \left(N_{\max }\right), X_{0}$ e $\lambda$ em bins de $X_{\max }$ e energia. A figura 4.19 mostra alguns exemplos das distribuições desses parâmetros para um intervalo específico de $X_{\max }$ e energia. As curvas vermelhas são funções gaussianas ajustadas às distribuições desses parâmetros. A etapa seguinte é parametrizar os dois parâmetros das gaussianas ajustadas (a média $\mu$ e a variância $\sigma$ ) em função do $X_{\max }$. A partir dessa parametrização é possível obter $\mu$ e $\sigma$ para qualquer valor de $X_{\max }$ em cada intervalo de energia. Em seguida, é feita uma interpolação linear entre os intervalos de energia com o objetivo de obter $\mu$ e $\sigma$ continuamente para qualquer valor de $X_{\max }$ e energia. Por fim os parâmetros $\log \left(N_{\max }\right), X_{0}$ e $\lambda$ para um dado valor de $X_{\max }$ e $\log (E)$ são obtidos randomicamente seguindo uma distribuição de probabilidades gaussiana com parâmetros $\mu\left(X_{\max }, \log (E)\right)$ e $\sigma\left(X_{\max }, \log (E)\right)$.

A figura 4.20 mostra a parametrização de $\mu$ e $\sigma$ em função de $X_{\max }$ para $\log \left(N_{\max }\right), X_{0}$ e $\lambda$, referente a um intervalo de energia específico. A curva vermelha é um polinômio de segundo grau ajustado ao gráfico. Os intervalos de $X_{\max }$ foram definidos em tamanhos variáveis com o objetivo de manter um número suficiente de eventos em cada bin para que o ajuste da função gaussiana fosse confiável. Nas tabelas 4.1, 4.2 e 4.3 estão mostrados os coeficientes obtidos por essa parametrização. Na figura 4.21 mostramos gráficos bidimensionais de $\mu$ e $\sigma$ para os três parâmetros em função de $\log (E / \mathrm{eV})$ e $X_{\max }$. As retas pretas mostram os limites dos valores de $X_{\max }$ que foram utilizados para construir a matriz resposta.

A partir das funções mostradas na figura 4.21 foi possível simular chuveiros cobrindo todas as regiões de $X_{\max }$ com a mesma amostragem. Isso pode ser observado na figura 4.22, que mostra um exemplo de uma matriz resposta construída utilizando este novo método, para um intervalo de energia. As figuras 4.23 e 4.24 mostram as matrizes resposta para todos os bins de energia.

Pela figura 4.19 fica evidente que os parâmetros $\log \left(N_{\max }\right), X_{0}$ e $\lambda$ não podem ser descritos exatamente através de funções gaussianas, e portanto esse ajuste funciona apenas como uma aproximação. Além disto, o procedimento descrito acima determina $\log \left(N_{\max }\right)$, $X_{0}$ e $\lambda$ independentemente, sem levar em conta qualquer correlação entre esses parâmetros. Utilizando esse mesmo conjunto de simulações descrito nesta seção, é possível observar uma 


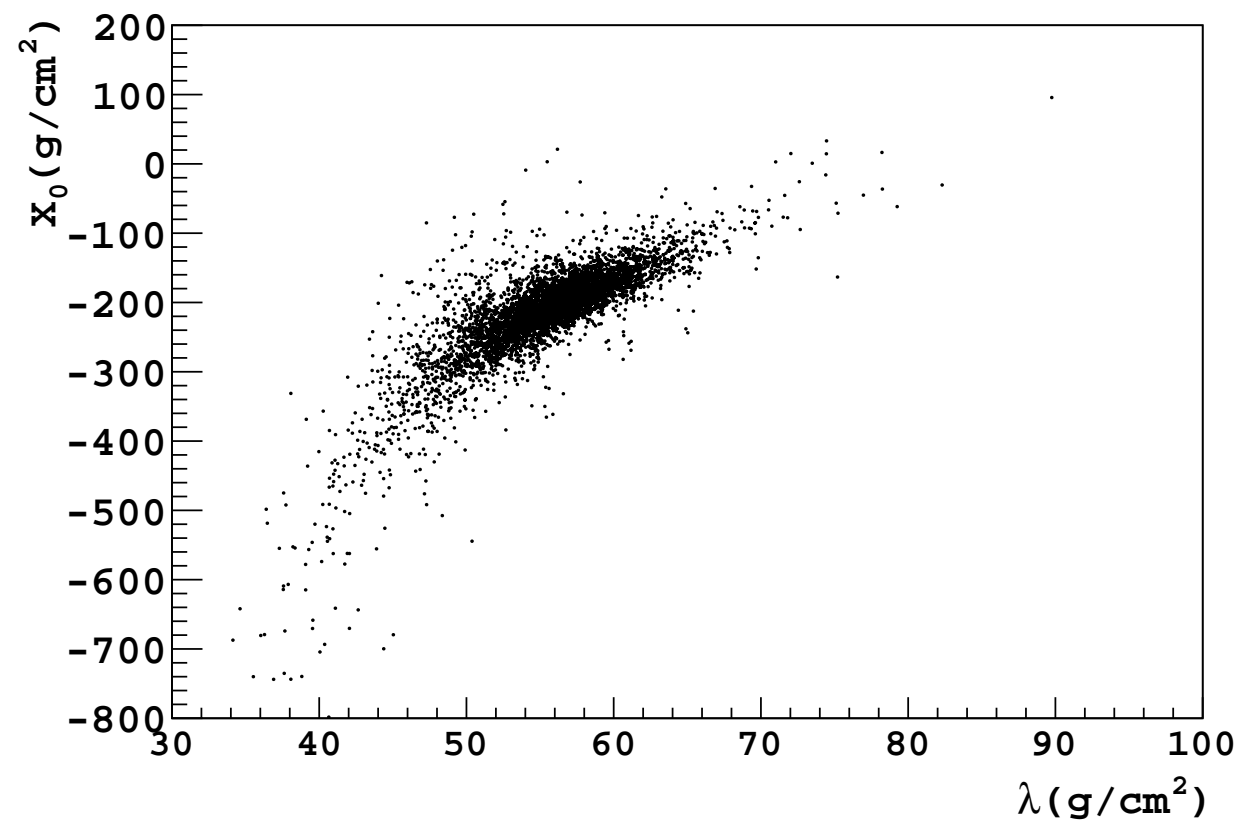

Figura 4.2 - Correlação entre $X_{0}$ e $\lambda$ para chuveiro simulados com energia igual a $10^{18.6} \mathrm{eV}$. Fonte: Imagem elaborada pelo autor.

correlação significativa entre os parâmetros $X_{0}$ e $\lambda$ para um valor fixo de energia (figura 4.2). Isto significa novamente que o algoritmo utilizado neste trabalho para gerar as matrizes resposta trata a questão simplificadamente, desprezando informações que podem ser importantes para outras aplicações.

Entretanto, no contexto do problema abordado aqui, o objetivo do método não é realizar uma descrição completa dos chuveiros atmosféricos medidos pelo Observatório Pierre Auger, mas sim construir de maneira confiável a matriz resposta do detector. Para essa aplicação, a posição do $X_{\max }$ é muito mais relevante do que os detalhes sobre a forma dos chuveiros. Por este motivo acreditamos que as aproximações efetuadas durante a parametrização, o modelo hadrônico escolhido e o conjunto de simulações utilizado não são fatores que influenciam fortemente as informações contidas na matriz.

\subsection{Testes de consistência}

Como foi dito anteriormente, a aplicação dos métodos de deconvolução geram soluções aproximadas que são fisicamente aceitáveis, entretanto, para isto são necessárias algumas informações a priori sobre as características esperadas das soluções. Além disto, informações 
obtidas através de modelos computacionais são essenciais para o funcionamento dos métodos, como acontece, por exemplo, na construção da matriz de resposta. Todos esse fatos podem enviesar os resultados dos métodos de deconvolução. Nesta seção, serão apresentados testes de consistência implementados com o objetivo de avaliar os possíveis efeitos citados acima.

Os testes de consistência baseiam-se na aplicação dos métodos de deconvolução em conjuntos de chuveiros simulados em que os valores de $X_{\max }$ verdadeiros e medidos são conhecidos, e então comparar os parâmetros extraídos das distribuições deconvoluídas com os parâmetros extraídos das distribuições verdadeiras. A diferença entre esses dois resultados representa uma medida da intensidade do viés incluído pelo processo de deconvolução. Neste trabalho foram utilizados conjuntos de chuveiros simulados pelo CONEX $(30,31)$ e reconstruídos pelo Offline (95) no modo real Monte Carlo, o que significa que são reproduzidas as condições reais do detector (condições técnicas e atmosféricas). Os chuveiros foram gerados considerando como partículas primárias prótons e núcleos de ferro, e o modelo hadrônico utilizado foi Sibyll2.1 (93).

Nas figuras 4.3 e 4.4 , estão os gráficos de $\left\langle X_{\max }\right\rangle$ e de $R M S_{X_{\max }}$ em função da energia, para o conjunto de simulações de próton e de núcleos de ferro após a aplicação dos métodos de deconvolução comparados com os valores reais. A figura 4.5 mostra as diferenças entre os valores de $\left\langle X_{\max }\right\rangle$ e $R M S_{X_{\max }}$ deconvoluídos e os valores reais para os dois conjuntos de simulações utilizados, que estão indicados como $\Delta X_{\max }$ e $\Delta R M S_{X_{\max }}$, respectivamente.

Notamos nas figuras 4.3 e 4.4 que o valor absoluto máximo de $\Delta X_{\max }$ e $\Delta R M S_{X_{\max }}$ é da ordem de $4 \mathrm{~g} / \mathrm{cm}^{2}$. Este valor é significantemente menor que as incertezas sistemáticas envolvidas na reconstrução do $X_{\max }$ (ver seção 4.4.3). Além disso, podemos notar que os valores de $\Delta X_{\max }$ e $\Delta R M S_{X_{\max }}$ oscilam em torno de 0 , sem apresentar um comportamento sistemático. Por estes motivos, concluímos que o resultado dos testes de consistência são suficientes para validar os algoritmos de deconvolução implementados neste trabalho.

\subsection{Análise de dados}

\subsubsection{Seleção dos dados}

Os dados utilizados neste trabalho foram medidos de forma híbrida pelo Observatório Pierre Auger entre 04/12/2004 e 31/12/2012. O limite mínimo de energia para os eventos 


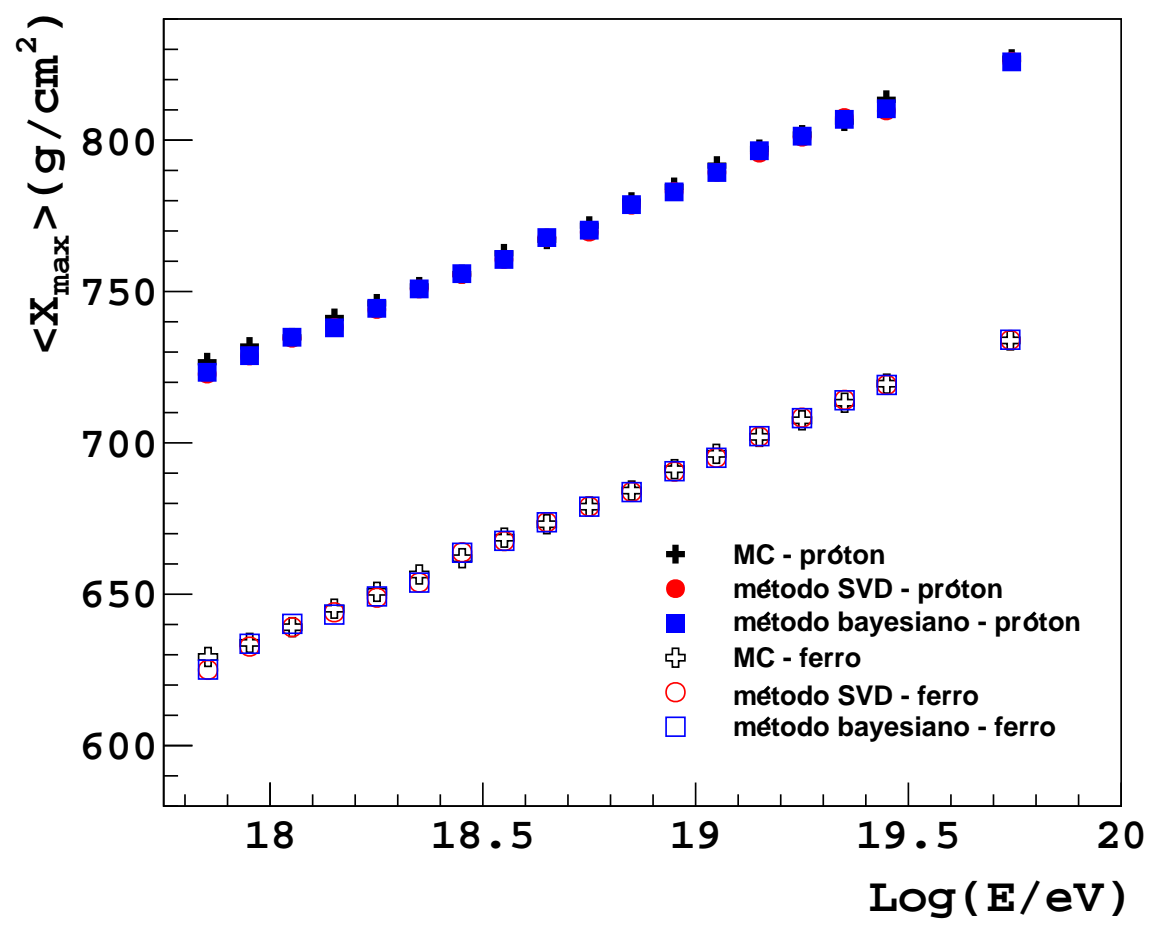

Figura 4.3 - Teste de consistência para $\left\langle X_{\max }\right\rangle$. Estão mostrados os valores de $\left\langle X_{\max }\right\rangle$ para as distribuição reais e deconvoluídas pelos dois métodos e para dois tipos de primários diferentes, prótons e núcleos de ferro. Fonte: Imagem elaborada pelo autor.

selecionados foi de $10^{17.8} \mathrm{eV}$. O número total de eventos selecionados foi 43704 . Os eventos foram divididos em intervalos com largura $\Delta \log (E / \mathrm{eV})=0.1$ entre $10^{17.8} \mathrm{eV}$ e $10^{19.5} \mathrm{eV}$. Os 55 eventos com energia acima de $10^{19.5} \mathrm{eV}$ foram aglutinados em um único bin. O evento mais energético selecionado tem energia de $8.21 \times 10^{19.0} \mathrm{eV}$. Foram aplicados cortes de qualidade básicos para eliminar eventos que não puderam ser bem reconstruídos. Abaixo estão listados os cortes, mais detalhes podem ser encontrados na referência (3).

Campo de visão: seleciona eventos em que o $X_{\max }$ medido está dentro do campo de visão dos telescópios.

Qualidade do ajuste: seleciona chuveiros em que o valor de $\chi^{2}$ por grau de liberdade para o ajuste da função Gaisser-Hillas ao perfil longitudinal é menor que 2.25.

Extensão do perfil: seleciona eventos que o perfil longitudinal medido tenha um comprimento maior que $300 \mathrm{~g} / \mathrm{cm}^{2}$.

Continuidade no perfil: seleciona eventos que não contenham intervalos no perfil medido maiores que $20 \%$ do comprimento total.

A figura 4.6 mostra o número de eventos para cada intervalo de energia nos dois casos: 


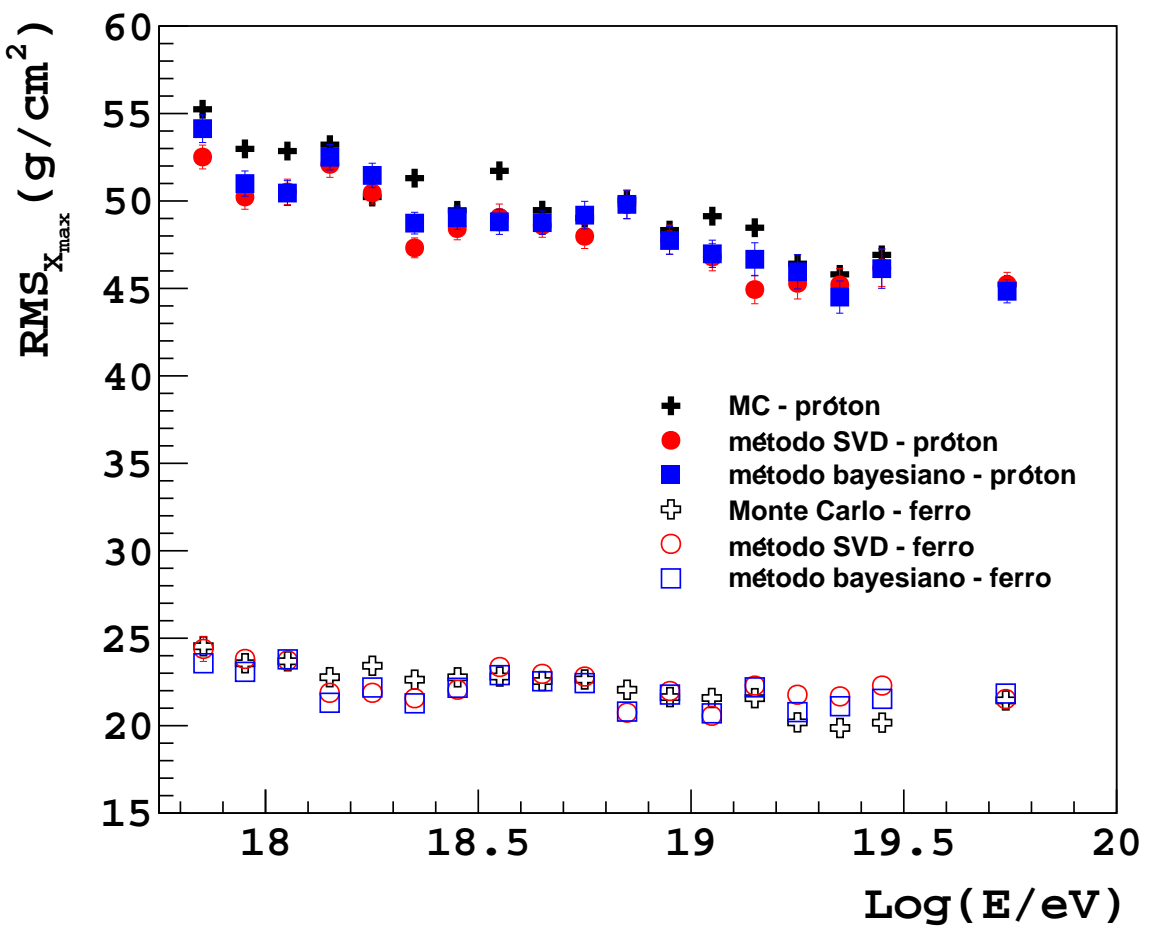

Figura 4.4 - Teste de consistência para $R M S_{X_{\max }}$. Estão mostrados os valores de $R M S_{X_{\max }}$ para as distribuição reais e deconvoluídas pelos dois métodos e para dois tipos de primários diferentes, prótons e núcleos de ferro. Fonte: Imagem elaborada pelo autor.

este trabalho, que aplica apenas cortes de qualidade, e na análise padrão, que aplica cortes de qualidade e cortes de fidelidade. O número de eventos por intervalo de energia para essa análise também está mostrado na tabela 4.4. A relação entre os números de eventos na presente análise e na análise padrão varia entre aproximadamente 2.5 no primeiro intervalo de energia, e 1.5, no intervalo de energia mais alta.

Entre os eventos detectados pelo Observatório Pierre Auger, existem os chamados eventos estéreos, que são medidos por mais de um telescópio de fluorescência. Nesses casos os valores da energia e do $X_{\max }$ medido são calculados a partir da média ponderada, definindo como peso o inverso ao quadrado da incerteza dos valores medidos por cada telescópio. Entre os eventos selecionados para esse trabalho 552 são estéreos.

\subsubsection{Incertezas estatísticas}

Os eventos medidos pelo Observatório Pierre Auger são independentes e portanto a correlação entre os bins da distribuição medida é nula. Isso significa que a sua matriz de covariância 


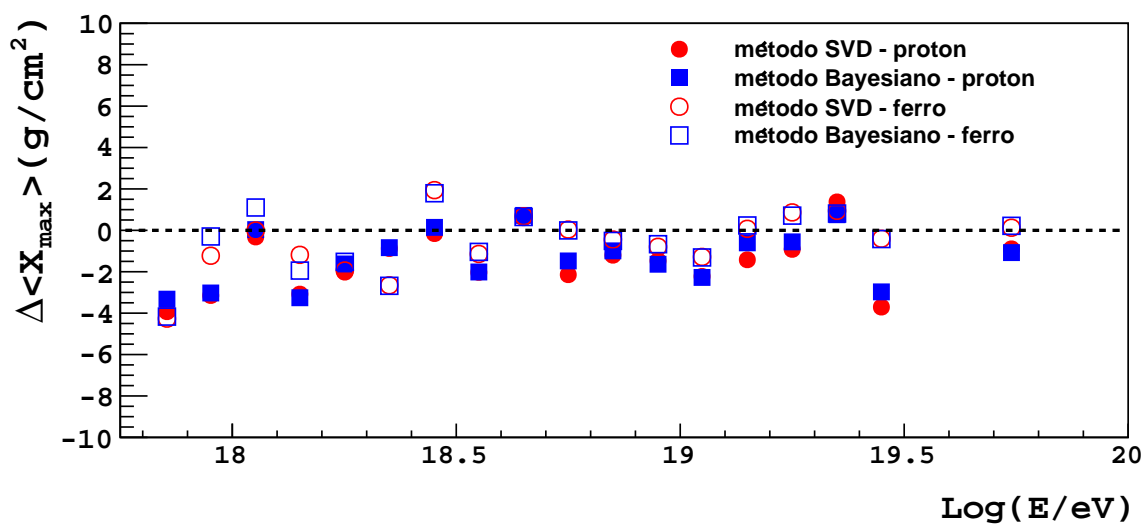

(a)

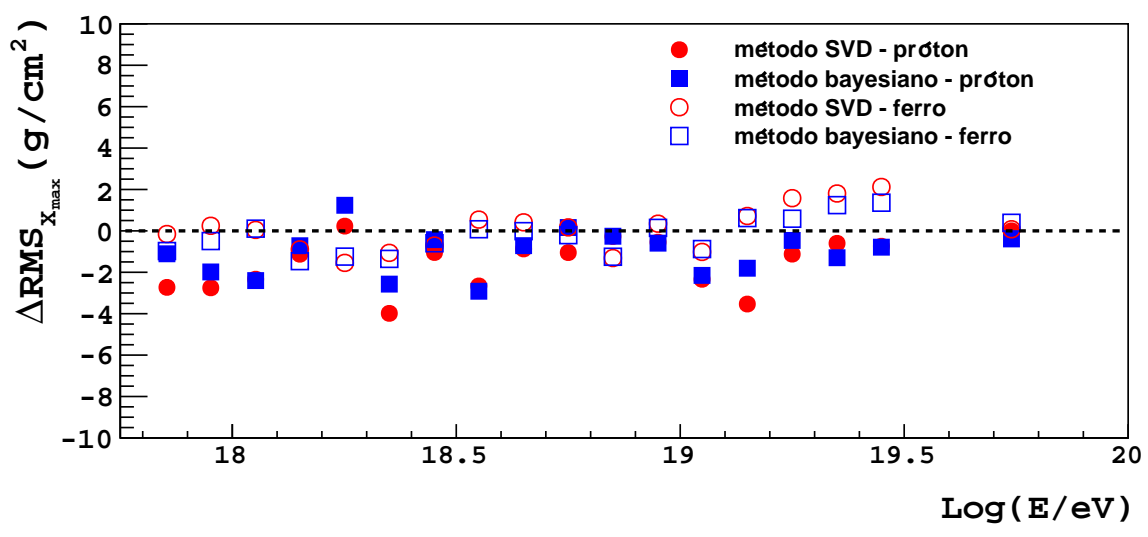

(b)

Figura 4.5 - Diferença entre os valores reais e deconvoluídos em função da energia para (a) $\left\langle X_{\max }\right\rangle$ e (b) $R M S_{X_{\max }}$. Fonte: Imagem elaborada pelo autor. 


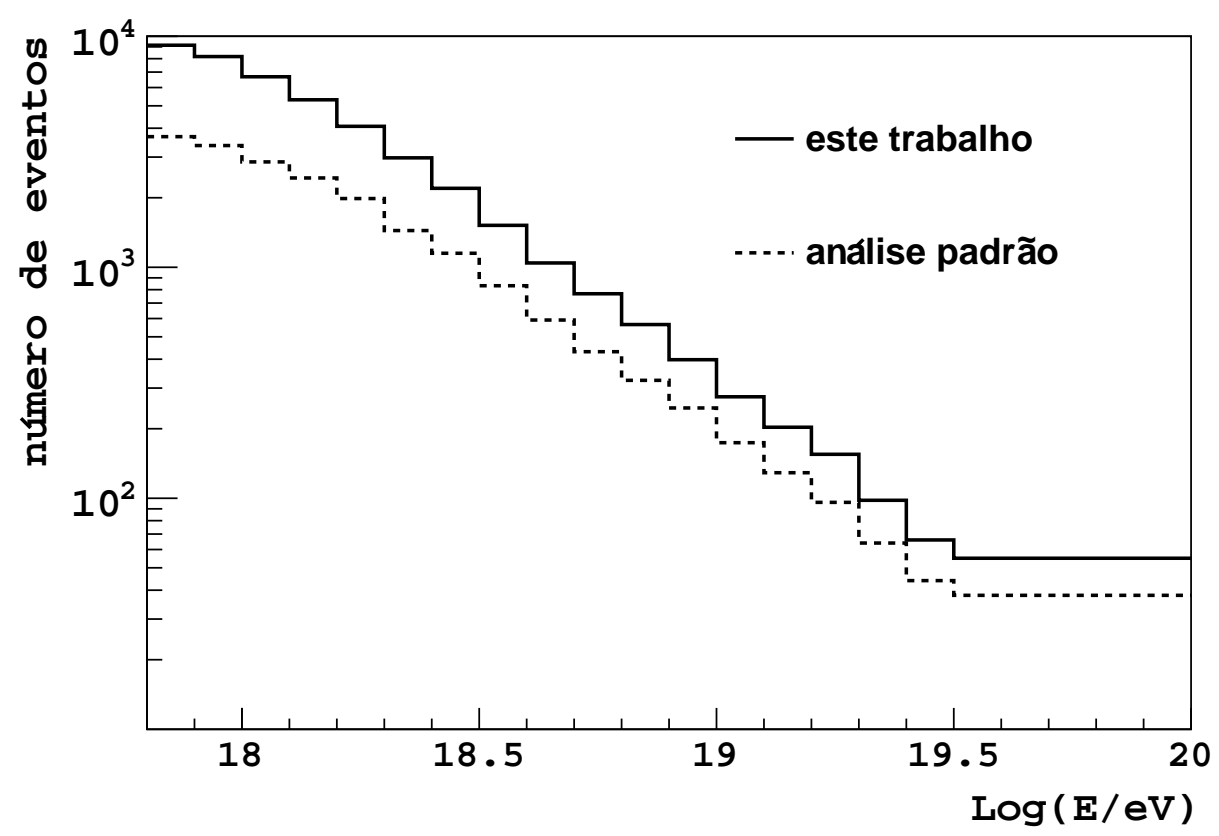

Figura 4.6 - Número de eventos selecionados para essa análise (somente cortes de qualidade) comparado com o número de eventos selecionados para a análise padrão (cortes de qualidade e fidelidade). Fonte: Imagem elaborada pelo autor.

é diagonal. Neste caso, o cálculo das incertezas estatísticas de $\left\langle X_{\max }\right\rangle$ e $R M S_{X_{\max }}$ é relativamente simples. Entretanto, o mesmo não é verdade para as distribuições deconvoluídas, devido ao fato do processo de deconvolução gerar correlações entre os bins. Nesta seção, apresentaremos os cálculos das incertezas estatísticas efetuados através de um chamado toy Monte Carlo, que leva em consideração tais correlações.

Primeiramente definiremos algumas grandezas importantes (as mesmas definições podem ser encontradas na referência (96)). O valor médio estimado de $X_{\max },\left\langle X_{\max }\right\rangle$, é definido como,

$$
\left\langle X_{\max }\right\rangle=\frac{1}{N} \sum_{i=1}^{N} X_{\max }^{i}
$$

em que $N$ é o número de eventos e $X_{\text {max }}^{i}$ 's são os valores de $X_{\max }$ de cada evento. A variância estimada de $X_{\max }$ é,

$$
\sigma_{X_{\max }}^{2}=\frac{1}{N-1} \sum_{i=1}^{N}\left(X_{\max }^{i}-\left\langle X_{\max }\right\rangle\right)^{2}
$$

A grandeza utilizada nesse trabalho para medir as flutuações dos $X_{\max }$ é o $R M S$, definido como,

$$
R M S_{X_{\max }}=\sqrt{\sigma_{X_{\max }}^{2}}
$$

No caso padrão, em que os bins das distribuições de $X_{\max }$ são descorrelacionados, as 
incertezas estatísticas de $\left\langle X_{\max }\right\rangle$ e $R M S_{X_{\max }}$ são dados por,

$$
\begin{gathered}
\sigma_{\left\langle X_{\max }\right\rangle}^{\prime 2}=\frac{R M S_{X_{\max }}}{\sqrt{N}} \\
\sigma_{R M S_{X_{\max }}^{\prime 2}}^{\prime 2}=\frac{1}{2 \cdot \sqrt{N} \cdot R M S_{X_{\max }}} \cdot \sqrt{m_{4}-\frac{N-3}{N-1}\left(R M S_{X_{\max }}\right)^{4}}
\end{gathered}
$$

em que novamente $N$ é o número de eventos e $m_{4}$ é o quarto momento da distribuição.

O toy Monte Carlo implementado neste trabalho consiste basicamente em aplicar os métodos de deconvolução a um número grande de distribuições que emulam as distribuições medidas, e então, a partir dos valores de $\left\langle X_{\max }\right\rangle$ e $R M S_{X_{\max }}$ das soluções obtidas, estimar suas variâncias e consequentemente as incertezas estatísticas. As distribuições medidas emuladas foram geradas aleatoriamente seguindo uma distribuição de probabilidade dada por uma função ajustada às distribuições medidas e contendo sempre o mesmo número de eventos original. A função utilizada para o ajuste é a proposta na referência (97). Os métodos de deconvolução foram aplicados a 1000 distribuições para cada intervalo de energia, e as incer-

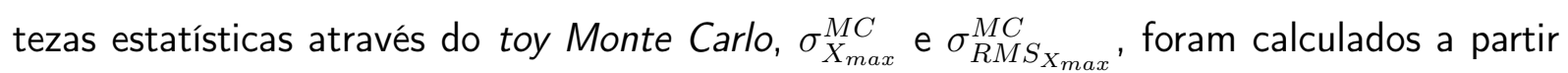
do $R M S$ das distribuições de $\left\langle X_{\max }\right\rangle$ e $R M S_{X_{\max }}$ das 1000 soluções deconvoluídas.

As figuras 4.7 e 4.8 mostram os resultados das incertezas estatísticas calculados através do toy Monte Carlo e através do cálculo padrão para os dois métodos de deconvolução, SVD e bayesiano. A incerteza padrão, indicada nas legendas por $\sigma^{\prime}$, é o valor médio das incertezas calculadas pelas equações 4.25 e 4.26 para as 1000 soluções obtidas. Pelos gráficos das figuras 4.7 e 4.8 nota-se que o aumento no valor da incerteza estatística devido às correlações geradas pelos métodos de deconvolução é significativo, principalmente em altas energias. Entretanto, apesar dessa diferença, o efeito da deconvolução não é suficiente para superar o efeito do aumento no número de eventos em relação a análise padrão. Nos resultados apresentados na seção 4.5 as incertezas estatísticas são as calculadas a partir do toy Monte Carlo, mostrados em 4.7 e 4.8

\subsubsection{Incertezas sistemáticas}

O cálculo do $X_{\max }$ a partir das medidas dos telescópios de fluorescência e dos tanques Cherenkov está sujeita a incertezas sistemáticas devido a várias fontes diferentes. Estudar e entender quais são as possíveis fontes e qual a intensidade da incerteza é uma tarefa complexa e de extrema importância para permitir a interpretação correta dos dados medidos. Parte 


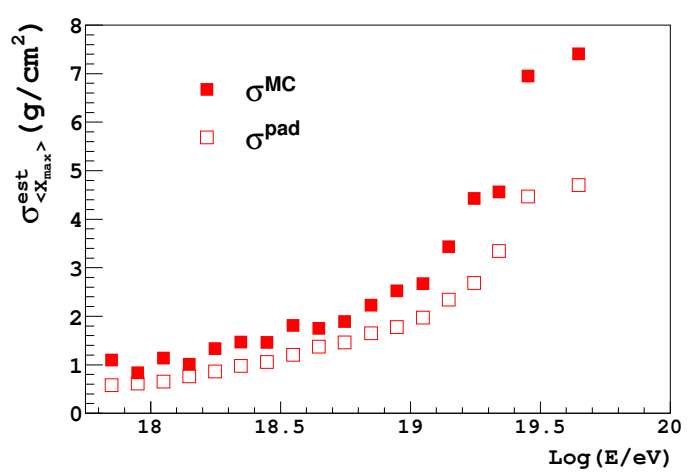

(a)

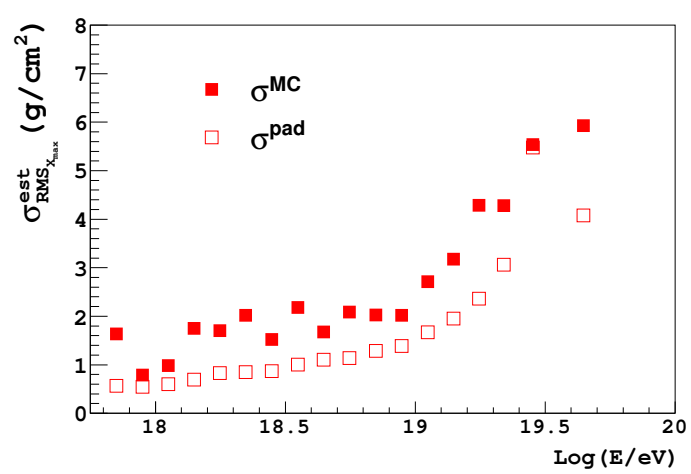

(b)

Figura 4.7 - Incertezas estatísticas em (a) $\left\langle X_{\max }\right\rangle$ e (b) $R M S_{X_{\max }}$ para o método SVD. A incerteza padrão está indicado como $\sigma^{\prime}$ e a incerteza calculada pelo toy Monte Carlo está indicado como $\sigma^{M C}$. Fonte: Imagem elaborada pelo autor.

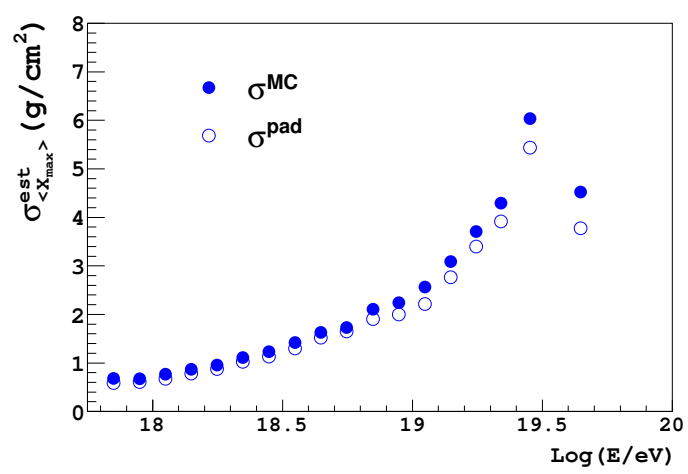

(a)

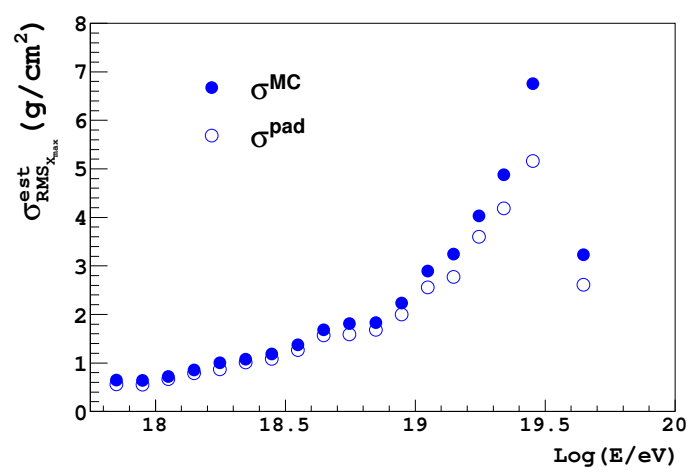

(b)

Figura 4.8 - Incertezas estatísticas em (a) $\left\langle X_{\max }\right\rangle$ e (b) $R M S_{X_{\max }}$ para o método bayesiano. A incerteza padrão está indicado como $\sigma^{\prime}$ e a incerteza calculada pelo toy Monte Carlo está indicado como $\sigma^{M C}$. Fonte: Imagem elaborada pelo autor. 
da colaboração do Observatório Pierre Auger se dedica a estudar essa questão, fazendo uso intenso de simulações de Monte Carlo, tanto dos chuveiros, como dos detectores. Uma descrição detalhada dos cálculos das incertezas sistemáticas associados as medidas de $X_{\max }$ pode ser encontrada na referência (3). Entretanto, apresentaremos neste trabalho a lista com as fontes de incertezas sistemáticas e um breve resumo a cerca de cada uma delas.

\section{Reconstrução do perfil longitudinal}

A principal fonte de incertezas sistemáticas na reconstrução do perfil longitudinal está associada a escolha da função Gaisser-Hillas para ajustar o perfil medido. Como foi explicado na seção 3.3.3, a função Gaisser-Hillas, como é utilizada neste contexto, possui 4 parâmetros livres para o ajuste, entretanto, em muitos eventos não existe informação suficiente no perfil medido para que o ajuste seja feito de maneira livre. Por esse motivo são definidos limites para os parâmetros $X_{0}$ e $\lambda$ com o objetivo de reduzir a liberdade do ajuste e permitir que esse procedimento funcione efetivamente para todos os eventos. O erro associado a esse processo é avaliado através de simulações, comparando os valores médios de $X_{\max }$ obtidos com e sem a imposição desses limites. Além disso, foi avaliado também a incerteza associada a escolha da função Gaisser-Hillas, uma vez que existem outras possíveis funções que poderiam ser utilizadas para descrever o perfil longitudinal. Essa etapa foi feita comparando os valores de $X_{\max }$ obtidos pelo ajuste e os valores reais de $X_{\max }$ obtidos através dos chuveiros simulados. A incerteza total associada ao ajuste da função Gaisser-Hillas é da ordem de $4-5 \mathrm{~g} / \mathrm{cm}^{2}$ para $\circ\left\langle X_{\max }\right\rangle$.

O algoritmo de reconstrução utilizado e as correções aplicadas a função de distribuição lateral também são fontes de incertezas, sendo que o primeiro contribui com uma incerteza da ordem de apenas $1 \mathrm{~g} / \mathrm{cm}^{2}$ e o segundo da ordem de $5 \mathrm{~g} / \mathrm{cm}^{2}$. A contribuição para a incerteza sistemática referente à reconstrução do perfil longitudinal está mostrada na figura 4.9 em função da energia.

\section{Calibração}

A principal fonte de incerteza referente às calibrações dos detectores tem origem no alinhamento do telescópio. As medidas na elevação dos espelhos, que são muito importante para determinar a profundidade do $X_{\max }$ medido, carregam uma incerteza natural da ordem de $0.1^{\circ}$, que ao ser propagado às medidas de $X_{\max }$, geram um erro sistemático da ordem de $4 \mathrm{~g} / \mathrm{cm}^{2}$, 
variável com a energia. A calibração dos pixels das câmeras dos telescópios de fluorescências e a sincronização temporal entre os detectores de superfície e de fluorescência também são fontes importantes e contribuem com erros da ordem de $1 \mathrm{~g} / \mathrm{cm}^{2}$ e $2 \mathrm{~g} / \mathrm{cm}^{2}$ respectivamente nas medidas de $X_{\max }$. Na figura 4.9 está mostrado o erro sistemático associado a calibração em função da energia.

\section{Atmosfera}

Entre os efeitos atmosféricos que influenciam sistematicamente a reconstrução dos chuveiros estão as propriedades dos aerossóis e o perfil de distribuição molecular. Em ambos os casos as informações a priori sobre tais propriedades atmosféricas contém uma incerteza natural do processo de medida. A incerteza sistemática incluído na medida de $X_{\max }$ por esses efeitos é calculado pela propagação dessas incertezas durante o processo de reconstrução. Outro fator atmosférico importante é a presença de nuvens, que podem funcionar como filtros enviesando principalmente chuveiros rasos, com $X_{\max }$ pequeno. Nesse caso, é possível estimar as incertezas sistemáticas utilizando os próprios dados, através da comparação dos valores médios de $X_{\text {max }}$ medidos em períodos sem nuvens e com nuvens.

Todos os efeitos atmosféricos citados (aerossóis, perfil molecular e nuvens) influenciam sistematicamente tanto as medidas de $\left\langle X_{\max }\right\rangle$ quanto as de $R M S_{X_{\max }}$. Na figura 4.9 estão mostrados essas contribuições. Em 4.9(b) fica claro que as incertezas sistemáticas em $R M S_{X_{\max }}$ são dominadas pelos efeitos atmosféricos, com uma pequena contribuição dos efeitos da reconstrução da energia.

\section{Energia}

A reconstrução da energia dos chuveiros sofrem de incertezas que podem influenciar sistematicamente os valores de $X_{\max }$ medidos. O principal fator é a chamada energia invisível (76). A incerteza sistemática nos valores de $\left\langle X_{\max }\right\rangle$ e $R M S_{X_{\max }}$ são $2.0 \mathrm{~g} / \mathrm{cm}^{2}$ e $0.6 \mathrm{~g} / \mathrm{cm}^{2}$ respectivamente, independentes da energia. 


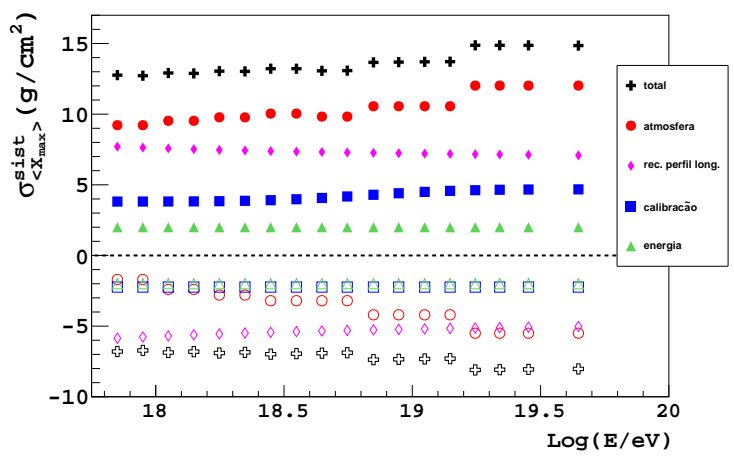

(a)

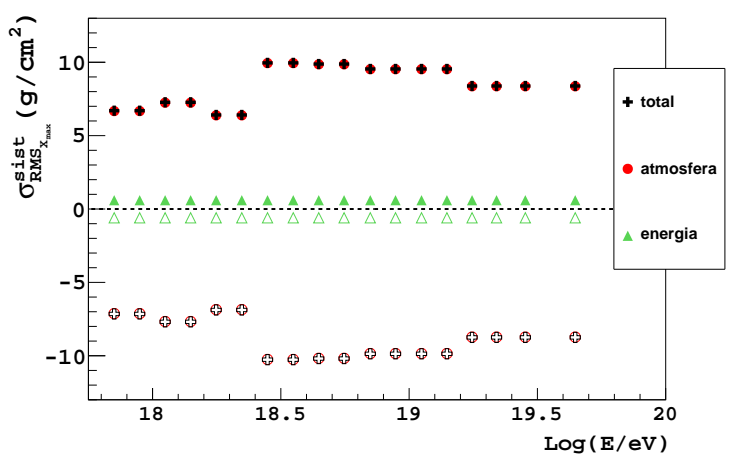

(b)

Figura 4.9 - Incertezas sistemáticas em (a) $\left\langle X_{\max }\right\rangle$ e (b) $R M S_{X_{\max }}$ em função da energia para cada fonte separadamente. Fonte: Imagem elaborada pelo autor.

\subsubsection{Número de iterações (somente método bayesiano)}

O número de iterações é um parâmetro importante do método iterativo bayesiano e deve ser escolhido de maneira a garantir a convergência da solução. Para avaliar o grau de convergência das soluções, definimos o parâmetro $\chi_{i, i-1}^{2}$ como,

$$
\chi_{i, i-1}^{2}=\frac{1}{N} \sum_{j=0}^{n}\left(\frac{X_{j}^{i}-X_{j}^{i-1}}{X_{j}^{i}}\right)^{2},
$$

em que $N$ é o número total de eventos, $n$ é o número de bins na distribuição, $i$ e $i-1$ são índices dos números de iterações e $j$ é o índice de cada bin da distribuição.

Calculando o parâmetro $\chi^{2}$ para cada iteração é possível determinar o número de iterações mínimo em que a convergência da solução é garantida para todos os bins de energia. A figura 4.10 mostra $\chi^{2}$ em função do número de iteração para todos os intervalos de energia. Podemos observar que a partir de 5 iterações, o valor de $\chi^{2}$ para todos os bins de energia é menor que 0.15. Sendo assim, o número de 5 iterações foi utilizado para obter todos os resultados desse trabalho. 


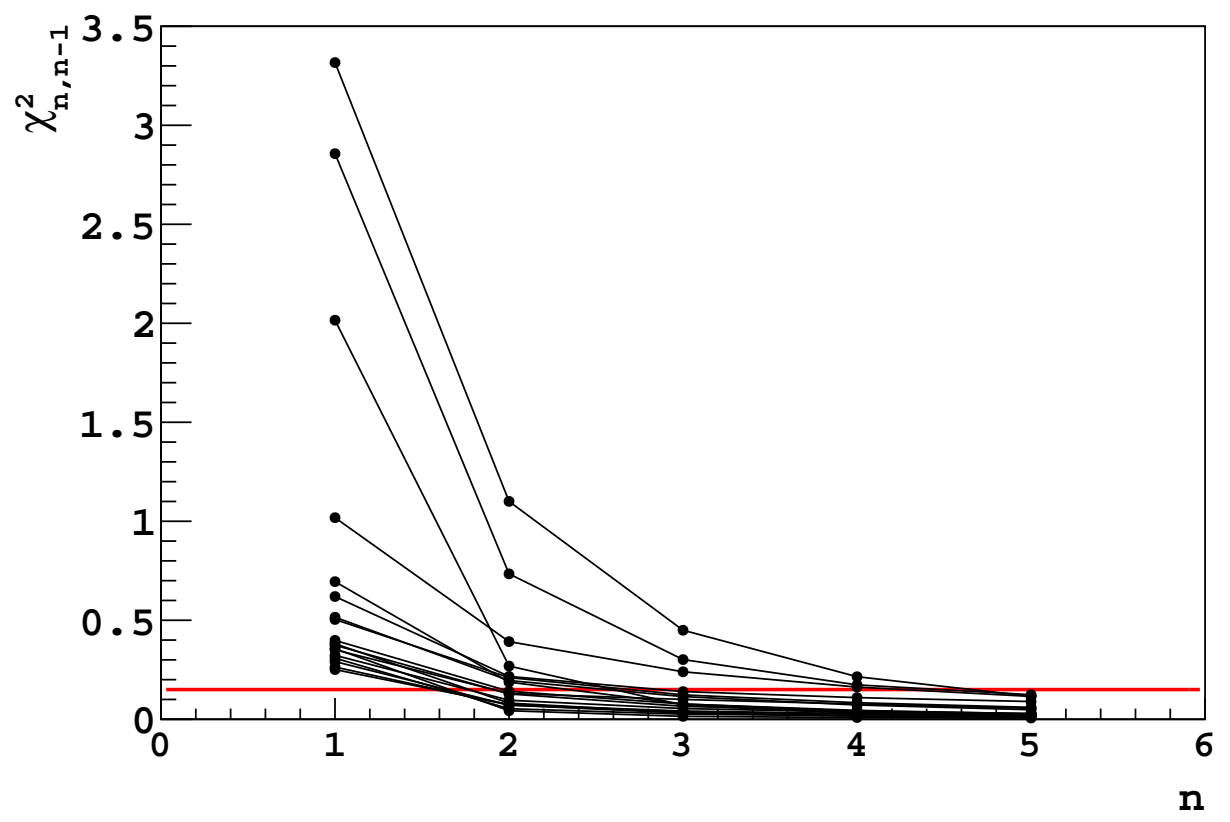

Figura $4.10-\chi_{n, n-1}^{2}$ em função do número de iterações $n$ para todos os bins de energia. A linha vermelha indica $\chi_{n, n-1}^{2}=0.15$. Fonte: Imagem elaborada pelo autor.

\subsection{Resultados e discussões}

Nessa seção, serão apresentados os resultados da aplicação dos métodos de deconvolução às distribuições de $X_{\max }$ medidas pelo Observatório Pierre Auger. Além das distribuições deconvoluídas de $X_{\max }$, serão avaliados a evolução com a energia de $\left\langle X_{\max }\right\rangle$ e $R M S_{X_{\max }}$. Em seguida utilizaremos a equação 2.5 para calcular $\langle\ln (A)\rangle$ como função da energia para diferentes modelos de interação hadrônica. Para finalizar, os resultados desse trabalho serão comparados com resultados de outros experimentos.

As distribuições de $X_{\max }$ deconvoluídas por ambos os métodos estão apresentadas na figura 4.25 e nas figuras 4.11 e 4.12 estão $\left\langle X_{\max }\right\rangle$ e $R M S_{X_{\max }}$ deconvoluídos em função da energia. Existe uma evidente concordância entre os dois métodos de deconvolução, tanto para os valores de $\left\langle X_{\max }\right\rangle$, quanto para o $R M S_{X_{\max }}$. As barras de erros das figuras 4.11 e 4.12 incluem apenas as incertezas estatísticas calculados na seção 4.4.2

Nas figuras 4.13 e 4.14 estão mostrados a evolução de $\left\langle X_{\max }\right\rangle$ e $R M S_{X_{\max }}$ com a energia para os resultados dos métodos de deconvolução comparados com os resultados da análise padrão, publicada no International Cosmic Rays Conference de 2013 (98) e predições de diferentes modelos hadrônicos. Nestes gráficos as barras de erro são apenas estatísticas, calculadas na seção 4.4.2. As incertezas sistemáticas estão mostradas na figura 4.15 como 


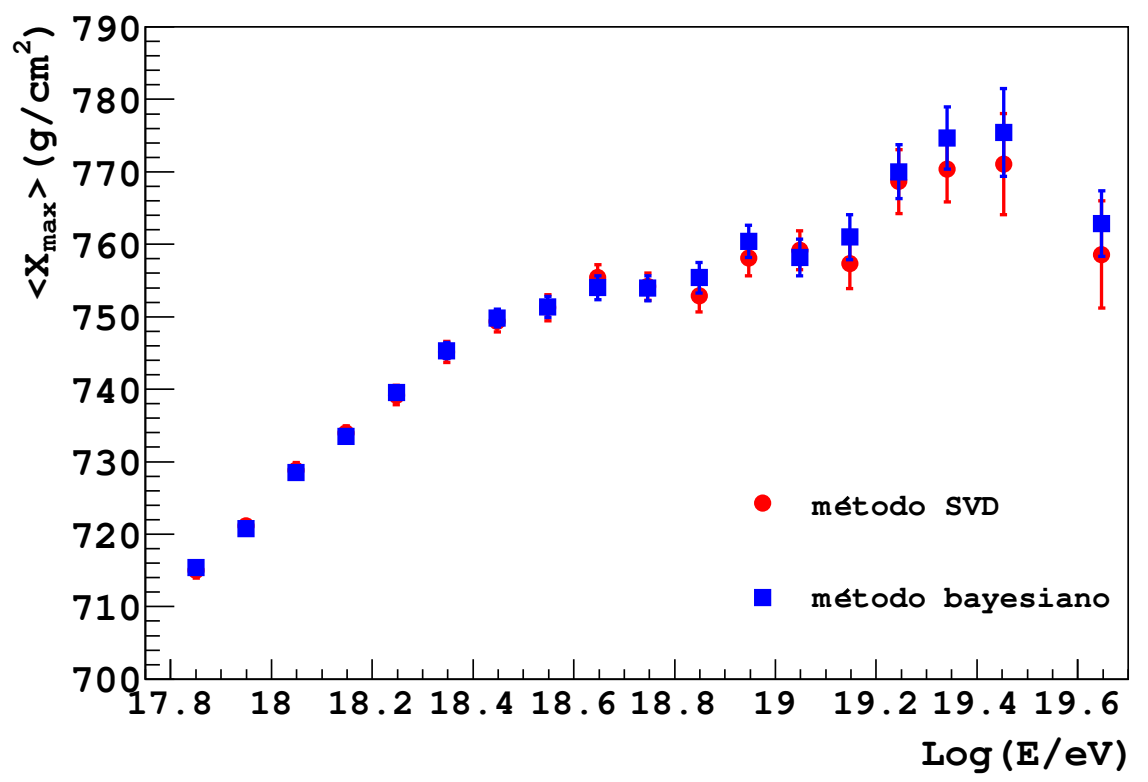

Figura 4.11 - $\left\langle X_{\max }\right\rangle$ em função da energia para os dois métodos de deconvolução. Fonte: Imagem elaborada pelo autor.

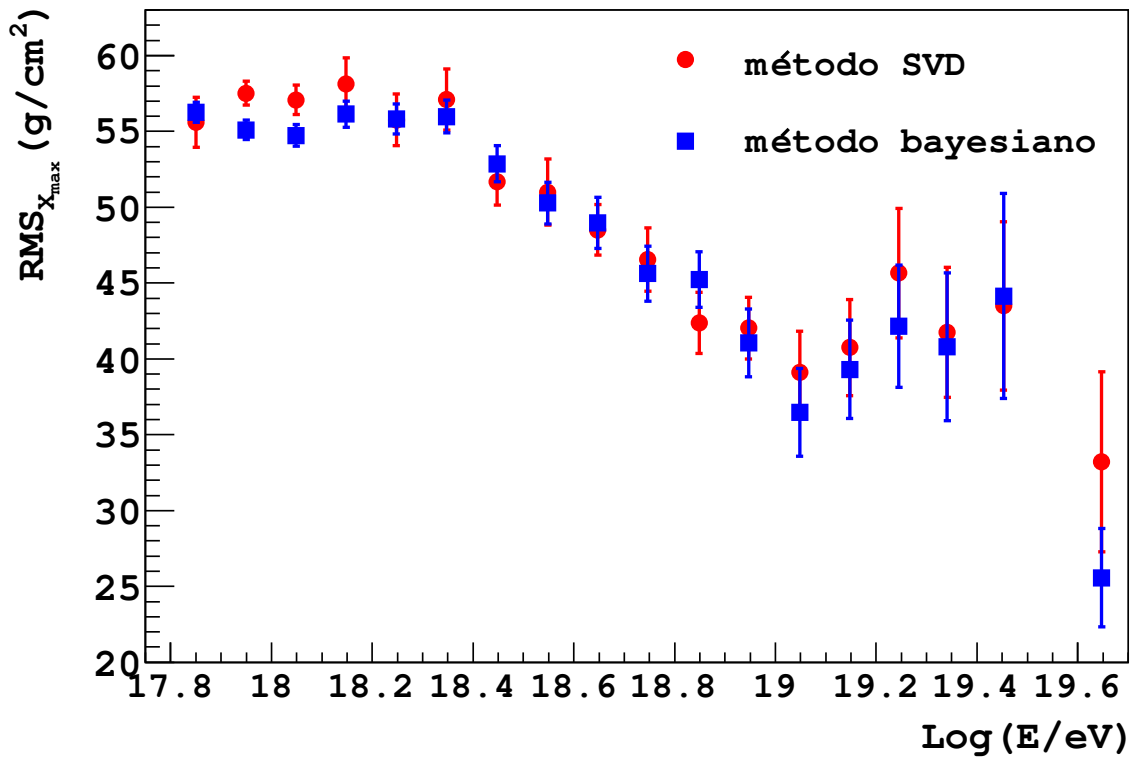

Figura 4.12 - $R M S_{X_{\max }}$ em função da energia para os dois métodos de deconvolução. Fonte: Imagem elaborada pelo autor. 


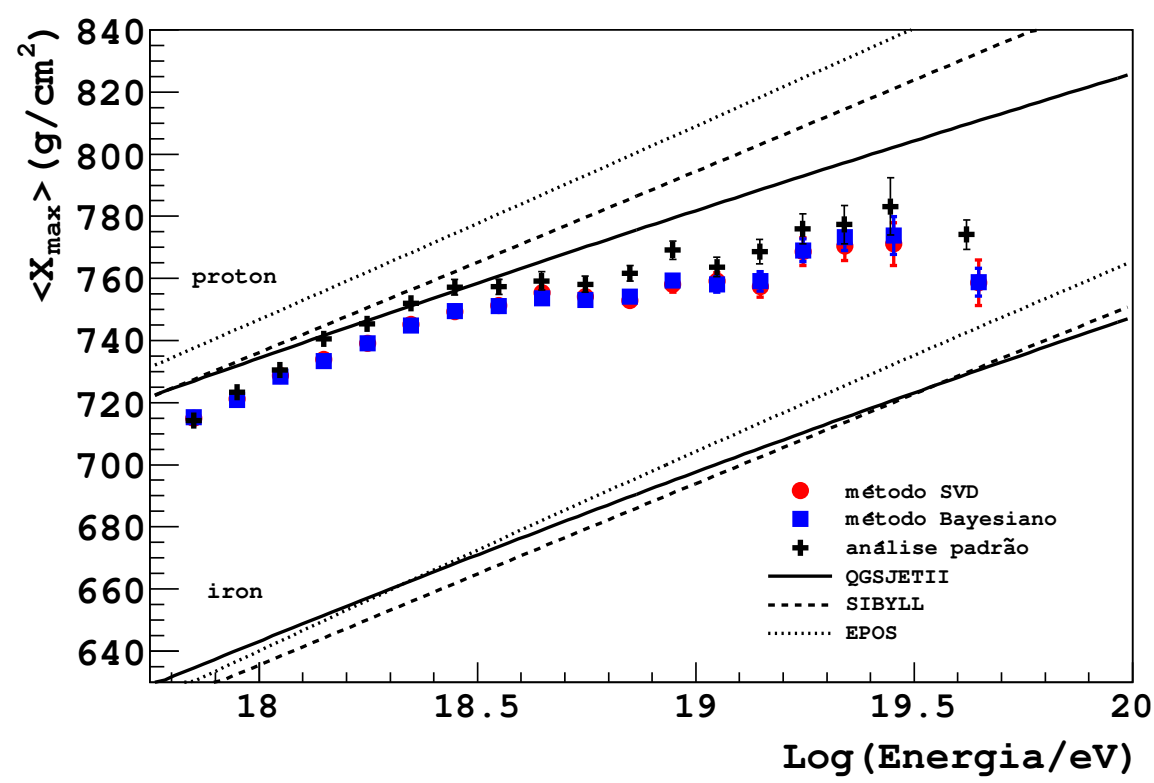

Figura $4.13-\left\langle X_{\max }\right\rangle$ em função da energia. As linhas mostram predições calculadas através de diferentes modelos de interação hadrônica para próton e núcleos de ferro. Fonte: Imagem elaborada pelo autor.

bandas preenchidas e correspondem aos erros descritos na seção 4.4.3

Comparando os valores de $\left\langle X_{\max }\right\rangle$ e $R M S_{X_{\max }}$ calculados a partir dos métodos de deconvolução com os valores de simulações obtidas através de modelos teóricos, é possível extrair informações sobre a composição primária média dos chuveiros medidos pelo Observatório Pierre Auger. A partir dos gráficos mostrados nas figuras 4.13 e 4.14 observamos que o comportamento de $\left\langle X_{\max }\right\rangle$ é muito parecido nos resultados dos métodos de deconvolução e na análise padrão. Em ambos os casos, assumindo como corretas as predições das simulações, podemos concluir que existem dois comportamentos diferentes: a) até aproximadamente $\log (E / e V) \sim 18.4$ os dados estão muito próximos das simulações de prótons, indicando uma predominância de partículas leves, b) a partir dessa energia, a inclinação do gráfico se altera, se aproximando das simulações de partículas mais pesadas. O comportamento observado nos gráficos de $R M S_{X_{\max }}$ em função da energia é muito parecido com o observado em $\left\langle X_{\max }\right\rangle$, mostrando uma alteração na inclinação a partir de $\log (E / e V) \sim 18.4$. Abaixo dessa energia os pontos também estão próximos das simulações de prótons e acima existe uma tendência a se aproximar de simulações de núcleos mais pesadas.

Apesar do comportamento geral dos resultados obtidos a partir dos métodos de deconvolução ser parecido com o observado na análise padrão, existem algumas vantagens da primeira com relação a segunda. Primeiramente, notamos que, como consequência do aumento no número de eventos, as barras de erro estatísticas são menores. Além disso, o número maior de 


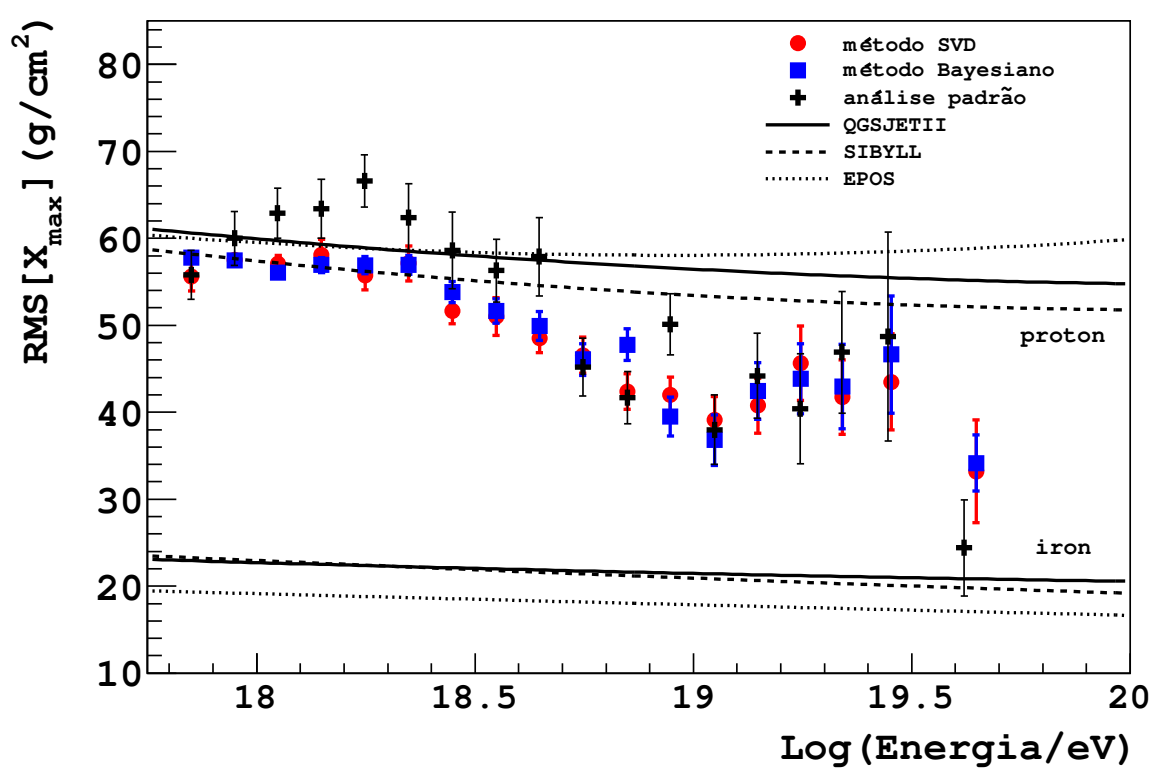

Figura 4.14 - $R M S_{X_{\max }}$ em função da energia. As linhas mostram predições calculadas através de diferentes modelos de interação hadrônica para próton e núcleos de ferro. Fonte: Imagem elaborada pelo autor.

eventos poderia permitir uma divisão com mais intervalos de energia. Isso deve ser realizado futuramente com o aumento dos eventos medidos pelo Observatório Pierre Auger. Outra diferença está no comportamento do $R M S_{X_{\max }}$ para energias menores que $10^{18.5} \mathrm{eV}$. Nesse intervalo de energia, notamos que, ao contrário da análise pelos métodos de deconvolução, os valores de $R M S_{X_{\max }}$ obtidos pela análise padrão estão acima dos valores obtidos pelas simulações de prótons, o que é difícil de entender frente aos modelos teóricos atuais.

A interpretação dos dados da taxa de elongação do ponto de vista da composição pode ser feita utilizando a equação 2.5, que permite calcular a massa logarítmica média $(\langle\ln (A)\rangle)$ através de $\left\langle X_{\max }\right\rangle$. Essa relação depende dos valores de $\left\langle X_{\max }\right\rangle_{p}$ e $\left\langle X_{\max }\right\rangle_{F e}$ calculados a partir dos modelos de interação hadrônica. Na figura 4.16 estão apresentados os valores de $\langle\ln (A)\rangle$ em função da energia para os resultados dos dois métodos de deconvolução, para três modelos de interação hadrônica diferentes: QGSJetll-04 (99, 100), EPOS-LHC (101), Sibyll2.1 (93). Observamos nesses gráficos com mais clareza o mesmo comportamento destacado acima com relação a $\left\langle X_{\max }\right\rangle$. Em energias abaixo de $\log (E / e V) \sim 18.4$, o valor de $\langle\ln (A)\rangle$ é quase constante e está abaixo de 1, o que significa partículas leves em média. Acima dessa energia a inclinação do gráfico se altera e o valor de $\langle\ln (A)\rangle$ tende a aumentar. Para os três modelos de interação hadrônica utilizados o comportamento de $\langle\ln (A)\rangle$ é muito parecido, a menos de diferenças sistemáticas menores que 0.5 .

As figuras 4.17 e 4.18 mostram a taxa de elongação calculada neste trabalho, a partir 


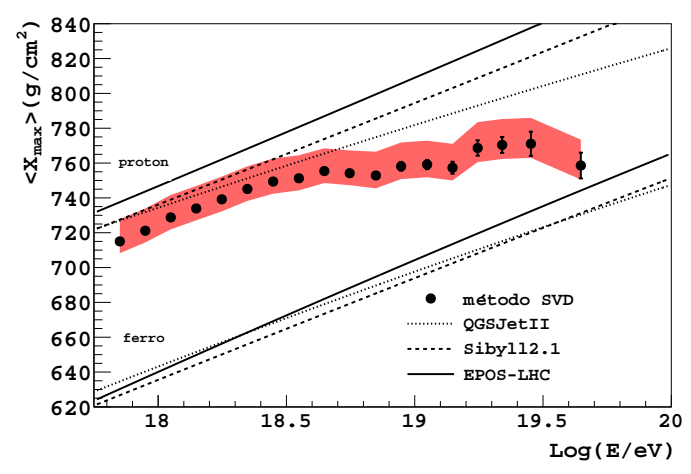

(a)

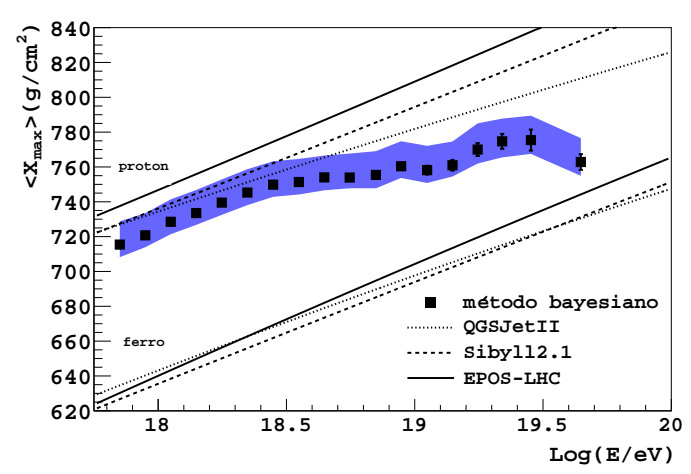

(c)

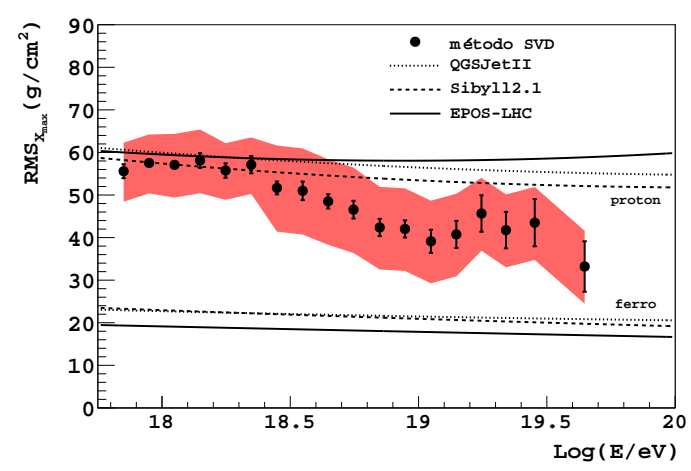

(b)

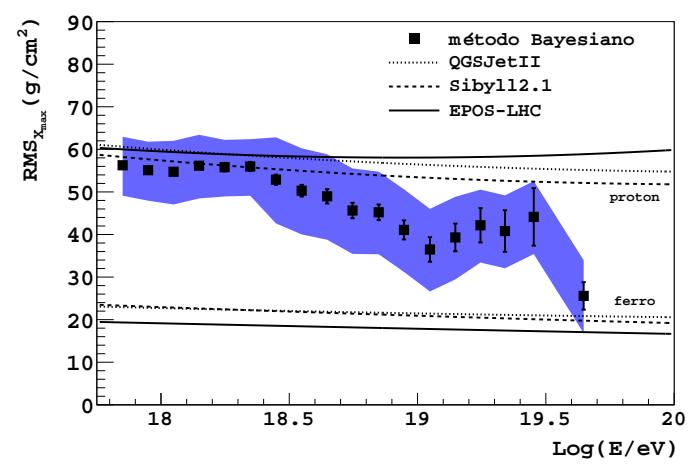

(d)

Figura 4.15 - (a) $\left\langle X_{\max }\right\rangle$ para o método SVD, (b) $R M S_{X_{\max }}$ para o método SVD, (c) $\left\langle X_{\max }\right\rangle$ para o método bayesiano e (d) $R M S_{X_{\max }}$ para o método bayesiano. As bandas preenchidas mostram as incertezas sistemáticas calculados na seção 4.4.3. Fonte: Imagens elaboradas pelo autor. 


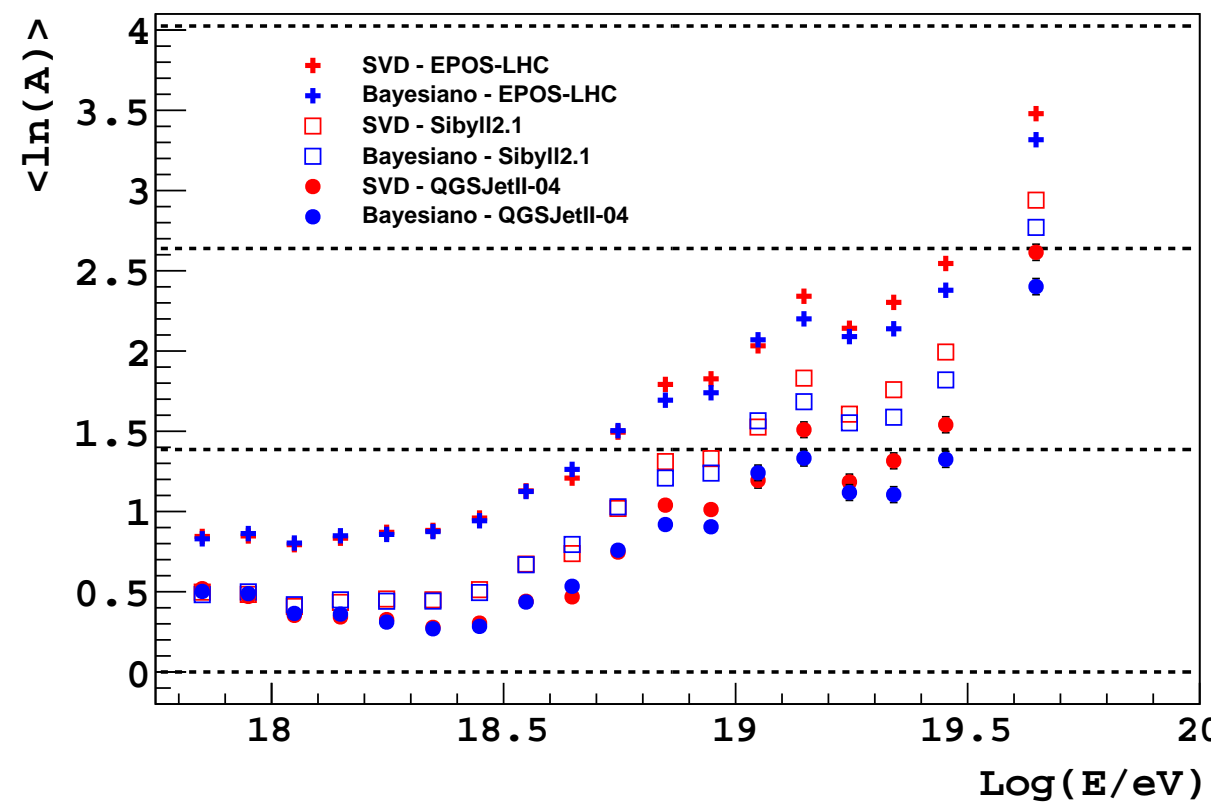

Fe

$\mathbf{N}$

He

\section{$\mathbf{p}$}

Figura 4.16 - $\operatorname{Ln}(A)$ em função da energia calculado através dos valores de $\left\langle X_{\max }\right\rangle$ deconvoluídos pelos métodos SVD e bayesiano. Fonte: Imagem elaborada pelo autor.

dos métodos de deconvolução, comparada com as taxas de elongação medidas por outros experimentos. Na figura 4.17 a escala de energia está ampliada, contendo resultados de experimentos que medem chuveiros com energia acima de $10^{15} \mathrm{eV}$. Na escala de energia que compreende os dados analisados nesse trabalho, mostrado na figura 4.18, observa-se que outros experimentos, como HiRes e o Telescope Array, medem chuveiros com valores de $\left\langle X_{\max }\right\rangle$ em média maiores que os medidos pelo Observatório Pierre Auger em altas energias $\left(>10^{18.5} \mathrm{eV}\right)$. Uma possível explicação de carácter astrofísico para essa diferença é que a composição das partículas medidas é diferente nos hemisférios norte e sul devido às diferentes fontes. Outra explicação possível seria de carácter experimental. Como o HiRes e o Telescope Array publicam seus resultados com os vieses experimentais inclusos, nos dados mostrados aqui foi realizada uma correção através da adição de um fator $\langle\Delta\rangle$, que é a diferença média entre os valores com viés e sem viés obtidos por simulações. Neste caso, os efeitos da detecção pode estar sendo mal estimada pelas simulações, o que estaria resultando nas diferenças observadas entre os experimentos. 


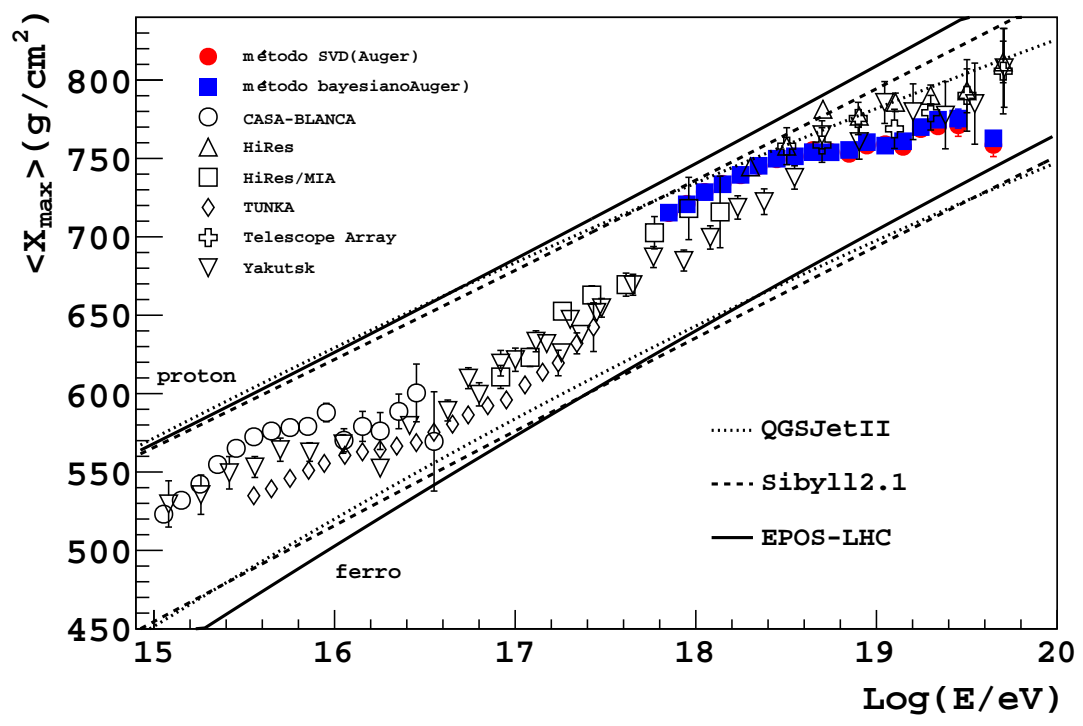

Figura $4.17-\left\langle X_{\max }\right\rangle$ em função da energia $\left(>10^{15.0}\right)$ para os resultados diferentes experimentos comparados com os resultados obtidos nesse trabalho. Os dados de outros experimentos foram extraídos da referência (27). Fonte: Imagem elaborada pelo autor.

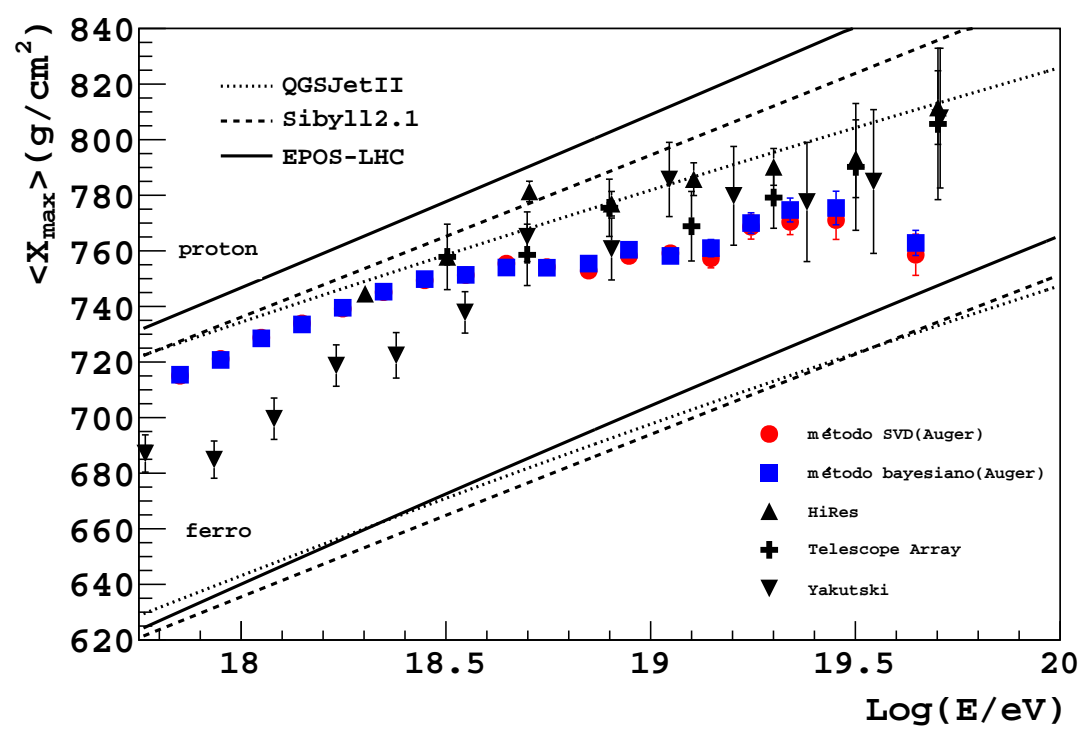

Figura $4.18-\left\langle X_{\max }\right\rangle$ em função da energia $\left(>10^{18.0}\right)$ para os resultados diferentes experimentos comparados com os resultados obtidos nesse trabalho. Os dados de outros experimentos foram extraídos da referência (27). Fonte: Imagem elaborada pelo autor. 
Tabela 4.1 - Parametrização de $\mu_{\log 10\left(N_{\max }\right)}$ e $\sigma_{\log 10\left(N_{\max }\right)}$ em função de $X_{\max }$. Os parâmetros mostrados são referentes as funções $\mu_{\log 10\left(N_{\max }\right)}=a_{0}+a_{1} \cdot X_{\max }+a_{2} \cdot X_{\max }^{2} \mathrm{e}$ $\sigma_{\log 10\left(N_{\max }\right)}=b_{0}+b_{1} \cdot X_{\max }+b_{2} \cdot X_{\max }^{2}$.

\begin{tabular}{|c|c|c|c|c|c|c|}
\hline $\mathrm{LgE}$ & $a_{0}$ & $a_{1}\left(\times 10^{4}\right)$ & $a_{2}\left(\times 10^{7}\right)$ & $b_{0}\left(\times 10^{2}\right)$ & $b_{1}\left(\times 10^{5}\right)$ & $b_{2}\left(\times 10^{8}\right)$ \\
\hline \hline 17.0 & 5.33 & -5.77 & 5.50 & 2.91 & -16.0 & 22.8 \\
\hline 17.1 & 5.48 & -7.27 & 6.57 & -7.66 & 19.1 & -6.57 \\
\hline 17.2 & 5.56 & -6.30 & 5.70 & -1.01 & -2.96 & 11.4 \\
\hline 17.3 & 5.76 & -9.50 & 8.18 & 0.79 & -8.51 & 15.3 \\
\hline 17.4 & 5.85 & -9.07 & 7.78 & -1.04 & -1.81 & 8.94 \\
\hline 17.5 & 5.95 & -8.78 & 7.49 & 0.50 & -7.26 & 13.5 \\
\hline 17.6 & 6.00 & -7.02 & 5.91 & -0.83 & -2.60 & 9.23 \\
\hline 17.7 & 6.15 & -8.49 & 7.01 & -8.10 & 20.0 & -8.48 \\
\hline 17.8 & 6.27 & -9.05 & 7.38 & -11.6 & 30.4 & -16.5 \\
\hline 17.9 & 6.35 & -8.37 & 6.70 & -2.65 & 2.50 & 5.04 \\
\hline 18.0 & 6.42 & -7.08 & 5.64 & -2.13 & 1.17 & 5.60 \\
\hline 18.1 & 6.46 & -5.38 & 4.30 & -0.89 & -1.99 & 7.32 \\
\hline 18.2 & 6.62 & -6.80 & 5.28 & -9.10 & 22.2 & -10.6 \\
\hline 18.3 & 6.75 & -7.62 & 5.84 & 0.36 & -5.44 & 9.32 \\
\hline 18.4 & 6.83 & -6.92 & 5.21 & -4.78 & 9.31 & -1.36 \\
\hline 18.5 & 6.83 & -3.89 & 2.97 & -0.63 & -1.89 & 5.95 \\
\hline 18.6 & 7.04 & -7.06 & 5.17 & -3.60 & 6.61 & -0.28 \\
\hline 18.7 & 7.09 & -5.48 & 3.96 & -7.31 & 17.3 & -8.06 \\
\hline 18.8 & 7.27 & -7.78 & 5.54 & -2.18 & 2.83 & 1.91 \\
\hline 18.9 & 7.31 & -5.81 & 4.06 & -5.86 & 13.0 & -5.20 \\
\hline 19.0 & 7.40 & -5.45 & 3.72 & -4.41 & 9.07 & -2.67 \\
\hline 19.1 & 7.46 & -4.34 & 2.91 & -5.22 & 11.7 & -4.93 \\
\hline 19.2 & 7.65 & -6.71 & 4.50 & -2.69 & 5.08 & -0.67 \\
\hline 19.3 & 7.70 & -5.27 & 3.46 & -2.66 & 4.96 & -0.65 \\
\hline 19.4 & 7.86 & -6.76 & 4.38 & -5.02 & 11.1 & -4.68 \\
\hline 19.5 & 7.81 & -2.66 & 1.57 & 2.98 & -10.3 & 9.54 \\
\hline 19.6 & 7.89 & -1.96 & 1.03 & 4.04 & -13.3 & 11.5 \\
\hline 19.7 & 8.02 & -2.66 & 1.48 & -0.84 & 0.28 & 2.02 \\
\hline 19.8 & 8.17 & -3.99 & 2.31 & 4.89 & -14.9 & 12.0 \\
\hline 19.9 & 8.09 & 0.96 & -0.99 & 8.36 & -23.8 & 17.6 \\
\hline 20.0 & 8.17 & 1.26 & -1.21 & 4.73 & -14.3 & 11.4 \\
\hline & & & & & & \\
\hline
\end{tabular}

Fonte: Tabela elaborado pelo autor. 
Tabela 4.2 - Parametrização de $\mu_{\lambda}$ e $\sigma_{\lambda}$ em função de $X_{\max }$. Os parâmetros mostrados são referentes as funções $\mu_{\lambda}=a_{0}+a_{1} \cdot X_{\max }+a_{2} \cdot X_{\max }^{2}$ e $\sigma_{\lambda}=b_{0}+b_{1} \cdot X_{\max }+b_{2} \cdot X_{\max }^{2}$.

\begin{tabular}{|c|c|c|c|c|c|c|}
\hline LgE & $a_{0}$ & $a_{1}\left(\times 10^{2}\right)$ & $a_{2}\left(\times 10^{5}\right)$ & $b_{0}$ & $b_{1}\left(\times 10^{3}\right)$ & $b_{2}\left(\times 10^{6}\right)$ \\
\hline 17.0 & 72.0 & 3.63 & -8.68 & -11.1 & 36.8 & -15.3 \\
\hline 17.1 & 58.3 & 7.89 & -11.9 & 1.75 & -8.85 & 24.0 \\
\hline 17.2 & 72.3 & 2.90 & -7.56 & 12.4 & -43.8 & 51.6 \\
\hline 17.3 & 42.5 & 11.8 & -14.2 & -4.67 & 7.34 & 12.9 \\
\hline 17.4 & 14.6 & 21.1 & -22.0 & -20.0 & 62.3 & -36.7 \\
\hline 17.5 & 35.5 & 14.2 & -16.3 & -32.0 & 98.0 & -63.5 \\
\hline 17.6 & 44.5 & 10.8 & -13.1 & -22.8 & 66.3 & -37.1 \\
\hline 17.7 & 52.1 & 8.16 & -10.9 & 3.95 & -17.6 & 27.8 \\
\hline 17.8 & 34.4 & 13.4 & -14.9 & -29.7 & 85.5 & -51.5 \\
\hline 17.9 & 9.23 & 20.8 & -20.2 & -42.2 & 121.0 & -77.6 \\
\hline 18.0 & 31.6 & 13.6 & -14.5 & -3.77 & 1.78 & 14.7 \\
\hline 18.1 & 26.9 & 14.9 & -15.4 & -44.3 & 125.0 & -78.7 \\
\hline 18.2 & 47.7 & 8.29 & -10.3 & -14.4 & 35.3 & -12.8 \\
\hline 18.3 & 42.9 & 9.42 & -10.9 & -0.16 & -6.65 & 17.6 \\
\hline 18.4 & 23.7 & 14.6 & -14.4 & -1.68 & -2.47 & 14.3 \\
\hline 18.5 & 51.9 & 6.19 & -8.17 & -4.82 & 6.41 & 7.51 \\
\hline 18.6 & 32.2 & 11.9 & -12.2 & -32.9 & 86.1 & -49.3 \\
\hline 18.7 & 27.1 & 12.8 & -12.6 & -13.9 & 30.4 & -9.08 \\
\hline 18.8 & 1.88 & 19.9 & -17.6 & -14.3 & 31.3 & -9.83 \\
\hline 18.9 & 6.44 & 18.0 & -15.9 & -7.95 & 12.3 & 3.83 \\
\hline 19.0 & 12.3 & 16.1 & -14.3 & -23.9 & 56.3 & -26.9 \\
\hline 19.1 & 41.6 & 7.85 & -8.61 & 2.48 & -15.3 & 21.4 \\
\hline 19.2 & 7.71 & 16.8 & -14.5 & -23.6 & 56.5 & -28.3 \\
\hline 19.3 & 19.8 & 13.2 & -11.9 & 22.8 & -70.1 & 57.5 \\
\hline 19.4 & -10.5 & 21.1 & -17.0 & -17.3 & 37.5 & -14.7 \\
\hline 19.5 & 46.5 & 5.41 & -6.25 & 5.83 & -23.2 & 24.7 \\
\hline 19.6 & 61.7 & 1.27 & -3.46 & 0.50 & -10.4 & 16.9 \\
\hline 19.7 & 28.2 & 9.77 & -8.85 & -12.7 & 24.2 & -6.07 \\
\hline 19.8 & -10.6 & 19.7 & -15.2 & 16.6 & -53.1 & 44.8 \\
\hline 19.9 & 67.8 & -1.13 & -1.39 & -0.01 & -8.26 & 14.2 \\
\hline 20.0 & 64.2 & -0.31 & -1.88 & 17.9 & -55.5 & 45.1 \\
\hline
\end{tabular}

Fonte: Tabela elaborado pelo autor. 
Tabela 4.3 - Parametrização de $\mu_{X_{0}}$ e $\sigma_{X_{0}}$ em função de $X_{\max }$. Os parâmetros mostrados são referentes as funções $\mu_{X_{0}}=a_{0}+a_{1} \cdot X_{\max }+a_{2} \cdot X_{\max }^{2}$ e $\sigma_{X_{0}}=b_{0}+b_{1} \cdot X_{\max }+b_{2} \cdot X_{\max }^{2}$.

\begin{tabular}{|c|c|c|c|c|c|c|}
\hline LgE & $a_{0}\left(\times 10^{-1}\right)$ & $a_{1}\left(\times 10^{2}\right)$ & $a_{2}\left(\times 10^{5}\right)$ & $b_{0}\left(\times 10^{-1}\right)$ & $b_{1}(\times 10)$ & $b_{2}\left(\times 10^{4}\right)$ \\
\hline 17 & 9.34 & -56.8 & 27.9 & 25.5 & -11.2 & 13.1 \\
\hline 17.1 & 13.8 & -74.6 & 45.3 & 8.48 & -5.21 & 7.74 \\
\hline 17.2 & -4.02 & -12.9 & -8.66 & 41.4 & -16.2 & 16.8 \\
\hline 17.3 & 10.7 & -64.1 & 35.1 & 20.6 & -9.4 & 11.1 \\
\hline 17.4 & -34.5 & 84.1 & -86.7 & 27.8 & -11.6 & 12.7 \\
\hline 17.5 & 8.05 & -51.1 & 20.2 & 3.27 & -3.5 & 5.97 \\
\hline 17.6 & -12.3 & 9.4 & -24.7 & 26.5 & -11.2 & 12.2 \\
\hline 17.7 & -23.2 & 45 & -54.7 & -8.2 & -0.353 & 3.74 \\
\hline 17.8 & -47.2 & 119 & -112 & 10.4 & -6.2 & 8.26 \\
\hline 17.9 & -67.7 & 180 & -158 & 19.6 & -8.88 & 10.1 \\
\hline 18 & -83.5 & 230 & -197 & 36.5 & -14.4 & 14.5 \\
\hline 18.1 & -77.4 & 207 & -178 & 9.76 & -6.2 & 8.18 \\
\hline 18.2 & -67.1 & 175 & -154 & 28.6 & -11.8 & 12.2 \\
\hline 18.3 & -2.61 & -20.4 & -6.56 & 0.722 & -3.28 & 5.69 \\
\hline 18.4 & -104 & 282 & -231 & 35.1 & -13.4 & 13.1 \\
\hline 18.5 & -21.9 & 35.7 & -48.8 & 31.7 & -12.4 & 12.3 \\
\hline 18.6 & -52.7 & 125 & -114 & 5.23 & -4.59 & 6.47 \\
\hline 18.7 & -96.5 & 250 & -204 & 28.5 & -11.5 & 11.5 \\
\hline 18.8 & -143 & 382 & -298 & 26.2 & -10.8 & 11 \\
\hline 18.9 & -114 & 293 & -230 & 49.6 & -17.7 & 16 \\
\hline 19 & -125 & 321 & -250 & 34.6 & -13.2 & 12.6 \\
\hline 19.1 & -41.9 & 88.2 & -87.6 & -20.9 & 2.32 & 1.65 \\
\hline 19.2 & -133 & 335 & -256 & 8.78 & -5.99 & 7.39 \\
\hline 19.3 & -161 & 411 & -306 & 23.4 & -10.1 & 10.2 \\
\hline 19.4 & -171 & 434 & -322 & 57.2 & -19.7 & 16.9 \\
\hline 19.5 & -106 & 254 & -197 & 3.21 & -4.4 & 6.1 \\
\hline 19.6 & -94.6 & 224 & -178 & 28.9 & -11.4 & 10.9 \\
\hline 19.7 & -100 & 233 & -181 & -36.6 & 5.74 & -0.457 \\
\hline 19.8 & -198 & 488 & -348 & 86 & -26.7 & 20.9 \\
\hline 19.9 & -14.1 & 3.16 & -29.2 & 36.6 & -13.3 & 11.9 \\
\hline 20 & -69 & 141 & -116 & -9.75 & -1.25 & 3.94 \\
\hline
\end{tabular}

Fonte: Tabela elaborado pelo autor. 
Tabela 4.4 - Resultados obtidos através do método SVD. Os valores de $\left\langle X_{\max }\right\rangle, R M S_{X_{\max }}$ e $\sigma$ estão em unidade de $\mathrm{g} / \mathrm{cm}^{2}$.

\begin{tabular}{|c|c|c|c|c|c|c|c|}
\hline$L g(\langle E\rangle)$ & $N$ & $\left\langle X_{\max }\right\rangle$ & $\sigma_{\text {estat }}^{\left\langle X_{\max }\right\rangle}$ & $\sigma_{\text {sist }}^{\left\langle X_{\max }\right\rangle}$ & $R M S_{X_{\max }}$ & $\sigma_{\text {estat }}^{\text {RMS }}$ & $\sigma_{\text {sist }}^{\text {RMS }}$ \\
\hline \hline 17.85 & 9148 & 715.0 & 1.101 & $+12.76-6.80$ & 55.60 & 1.64 & $+6.70-7.15$ \\
\hline 17.95 & 8170 & 721.1 & 0.837 & $+12.72-6.72$ & 57.52 & 0.79 & $+6.70-7.15$ \\
\hline 18.05 & 6680 & 728.8 & 1.140 & $+12.91-6.87$ & 57.09 & 0.98 & $+7.27-7.69$ \\
\hline 18.15 & 5310 & 733.9 & 1.014 & $+12.88-6.81$ & 58.11 & 1.75 & $+7.27-7.69$ \\
\hline 18.25 & 4076 & 739.2 & 1.333 & $+13.04-6.91$ & 55.76 & 1.70 & $+6.41-6.88$ \\
\hline 18.35 & 2977 & 745.1 & 1.465 & $+13.03-6.86$ & 57.12 & 2.02 & $+6.41-6.88$ \\
\hline 18.45 & 2197 & 749.4 & 1.460 & $+13.22-6.99$ & 51.67 & 1.52 & $+9.96-10.27$ \\
\hline 18.55 & 1519 & 751.3 & 1.808 & $+13.22-6.95$ & 50.99 & 2.18 & $+9.96-10.27$ \\
\hline 18.65 & 1044 & 755.4 & 1.751 & $+13.07-6.91$ & 48.50 & 1.67 & $+9.88-10.19$ \\
\hline 18.75 & 768 & 754.2 & 1.892 & $+13.08-6.88$ & 46.56 & 2.09 & $+9.88-10.19$ \\
\hline 18.85 & 565 & 752.9 & 2.227 & $+13.66-7.37$ & 42.38 & 2.02 & $+9.55-9.87$ \\
\hline 18.95 & 398 & 758.1 & 2.524 & $+13.68-7.35$ & 42.03 & 2.02 & $+9.55-9.87$ \\
\hline 19.05 & 275 & 759.2 & 2.671 & $+13.70-7.32$ & 39.12 & 2.72 & $+9.55-9.87$ \\
\hline 19.15 & 203 & 757.3 & 3.429 & $+13.71-7.30$ & 40.76 & 3.18 & $+9.55-9.87$ \\
\hline 19.25 & 155 & 768.6 & 4.428 & $+14.87-8.10$ & 45.66 & 4.28 & $+8.39-8.75$ \\
\hline 19.34 & 98 & 770.4 & 4.561 & $+14.87-8.08$ & 41.76 & 4.28 & $+8.39-8.75$ \\
\hline 19.45 & 66 & 771.1 & 6.950 & $+14.87-8.06$ & 43.50 & 5.54 & $+8.39-8.75$ \\
\hline 19.65 & 55 & 758.6 & 7.406 & $+14.85-8.03$ & 33.21 & 5.93 & $+8.39-8.75$ \\
\hline
\end{tabular}

Fonte: Tabela elaborada pelo próprio autor. 
Tabela 4.5 - Resultados obtidos através do método iterativo bayesiano. Os valores de $\left\langle X_{\max }\right\rangle$, $R M S_{X_{\text {max }}}$ e $\sigma$ estão em unidade de $\mathrm{g} / \mathrm{cm}^{2}$.

\begin{tabular}{|c|c|c|c|c|c|c|}
\hline$L g(\langle E\rangle)$ & $\left\langle X_{\max }\right\rangle$ & $\sigma_{\text {estat }}^{\left\langle X_{\max }\right\rangle}$ & $\sigma_{\text {sist }}^{\left\langle X_{\max }\right\rangle}$ & $R M S_{X_{\max }}$ & $\sigma_{\text {estat }}^{\text {RMS }}$ & $\sigma_{\text {sist }}^{\text {RMS }}$ \\
\hline \hline 17.85 & 715.4 & 0.682 & $+12.76-6.80$ & 56.26 & 0.647 & $+6.70-7.15$ \\
\hline 17.95 & 720.8 & 0.673 & $+12.72-6.72$ & 55.10 & 0.638 & $+6.70-7.15$ \\
\hline 18.05 & 728.5 & 0.768 & $+12.91-6.87$ & 54.74 & 0.723 & $+7.27-7.69$ \\
\hline 18.15 & 733.5 & 0.871 & $+12.88-6.81$ & 56.15 & 0.859 & $+7.27-7.69$ \\
\hline 18.25 & 739.5 & 0.959 & $+13.04-6.91$ & 55.82 & 1.003 & $+6.41-6.88$ \\
\hline 18.35 & 745.3 & 1.113 & $+13.03-6.86$ & 55.98 & 1.079 & $+6.41-6.88$ \\
\hline 18.45 & 749.8 & 1.233 & $+13.22-6.99$ & 52.87 & 1.188 & $+9.96-10.27$ \\
\hline 18.55 & 751.4 & 1.422 & $+13.22-6.95$ & 50.29 & 1.376 & $+9.96-10.27$ \\
\hline 18.65 & 754.0 & 1.633 & $+13.07-6.91$ & 48.96 & 1.682 & $+9.88-10.19$ \\
\hline 18.75 & 753.9 & 1.731 & $+13.08-6.88$ & 45.64 & 1.813 & $+9.88-10.19$ \\
\hline 18.85 & 755.4 & 2.108 & $+13.66-7.37$ & 45.22 & 1.834 & $+9.55-9.87$ \\
\hline 18.95 & 760.4 & 2.239 & $+13.68-7.35$ & 41.07 & 2.236 & $+9.55-9.87$ \\
\hline 19.05 & 758.2 & 2.562 & $+13.70-7.32$ & 36.48 & 2.895 & $+9.55-9.87$ \\
\hline 19.15 & 761.0 & 3.092 & $+13.71-7.30$ & 39.31 & 3.243 & $+9.55-9.87$ \\
\hline 19.25 & 770.0 & 3.708 & $+14.87-8.10$ & 42.16 & 4.029 & $+8.39-8.75$ \\
\hline 19.34 & 774.7 & 4.292 & $+14.87-8.08$ & 40.81 & 4.879 & $+8.39-8.75$ \\
\hline 19.45 & 775.4 & 6.034 & $+14.87-8.06$ & 44.14 & 6.755 & $+8.39-8.75$ \\
\hline 19.65 & 762.9 & 4.523 & $+14.85-8.03$ & 25.58 & 3.233 & $+8.39-8.75$ \\
\hline
\end{tabular}

Fonte: Tabela elaborada pelo próprio autor. 


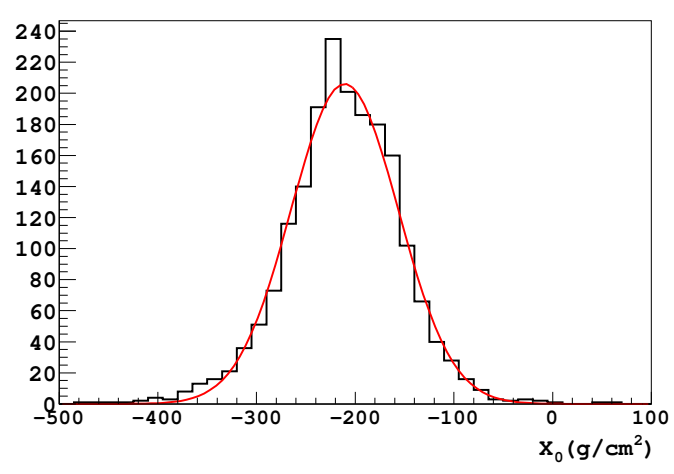

(a)

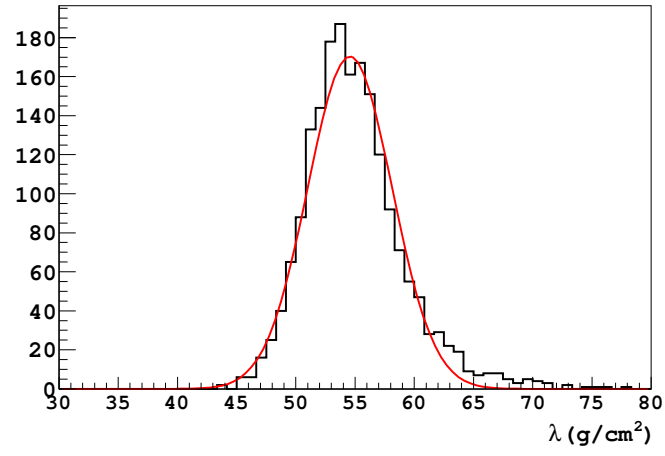

(b)

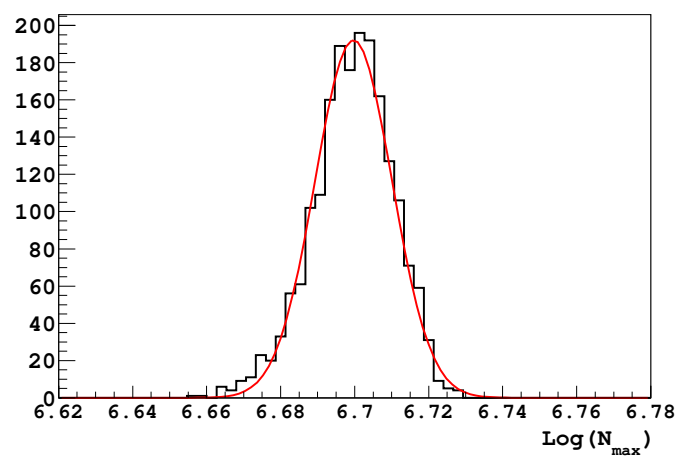

(c)

Figura 4.19 - Distribuições de (a) $X_{0}$, (b) $\lambda$ e (c) $\log \left(N_{\max }\right)$. O bin de energia é definido por $18.45<\log (E / \mathrm{eV})<18.5$ e o bin de $X_{\max }$ é definido por $700<X_{\max } /\left(\mathrm{g} / \mathrm{cm}^{2}\right)<$ 725. As curvas em vermelho mostram as funções gaussianas ajustadas às distribuições. Fonte: Imagens elaboradas pelo autor. 


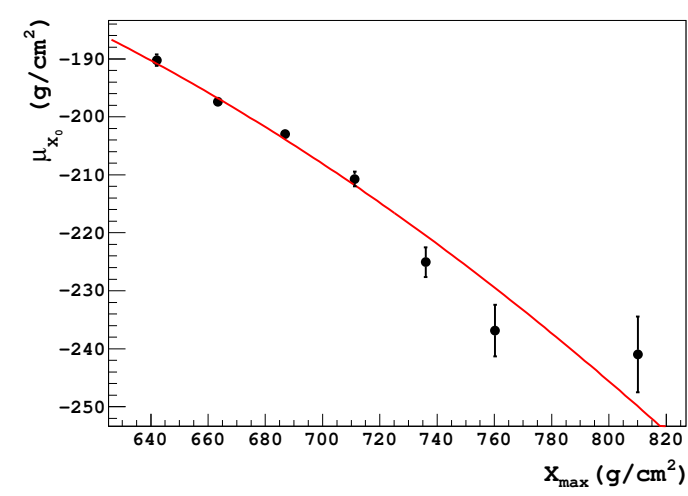

(a)

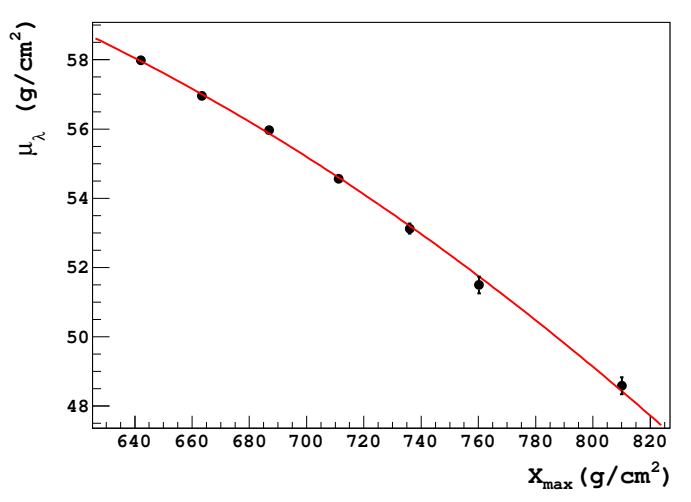

(c)

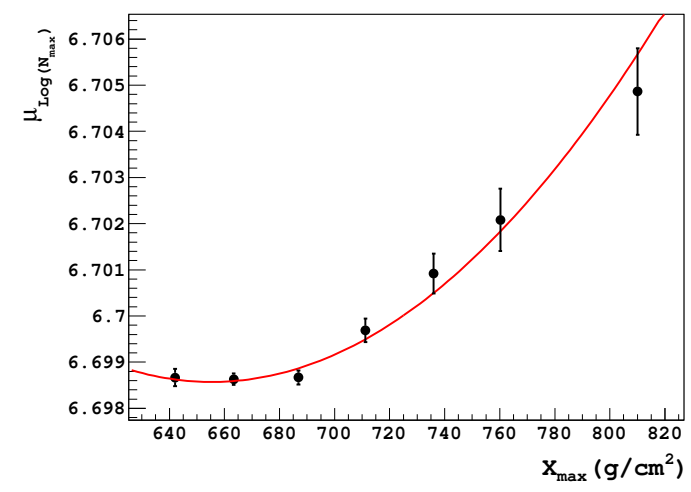

(e)

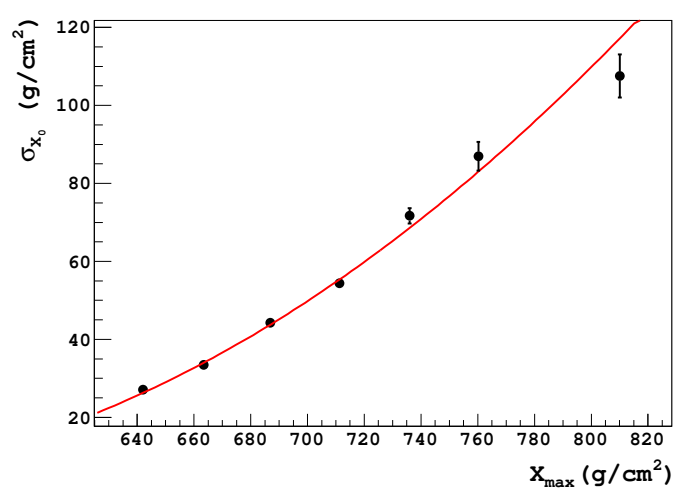

(b)

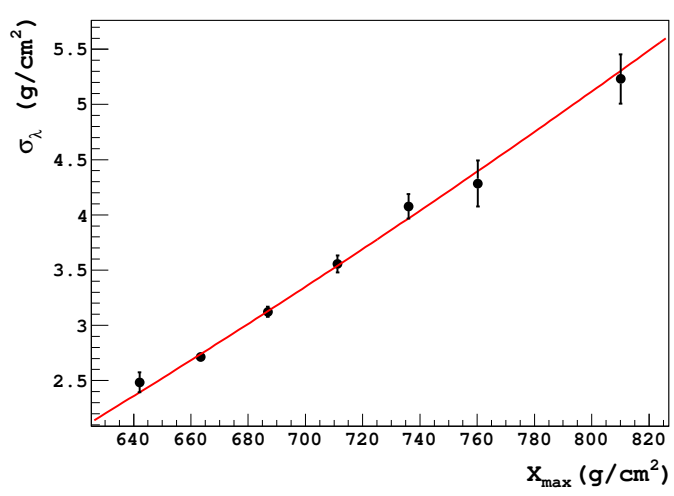

(d)

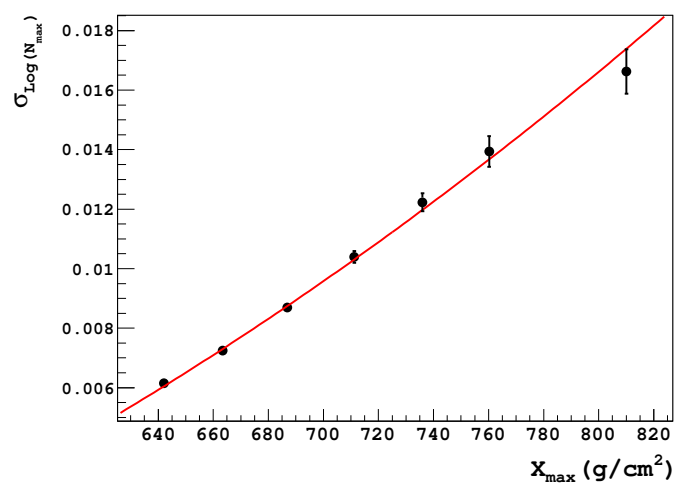

(f)

Figura 4.20 - Parametrização de (a) $\mu_{X_{0}}$, (b) $\sigma_{X_{0}}$, (c) $\mu_{\lambda}$, (d) $\sigma_{\lambda}$, (e) $\mu_{\log \left(N_{\max }\right)}$ e (f) $\sigma_{\log \left(N_{\max }\right)}$ em função de $X_{\max }$. O bin de energia é definido por $18.4<\log (E / \mathrm{eV})<18.5$. As curvas em vermelho são polinômios de segundo grau ajustados aos gráficos. Fonte: Imagens elaboradas pelo autor. 


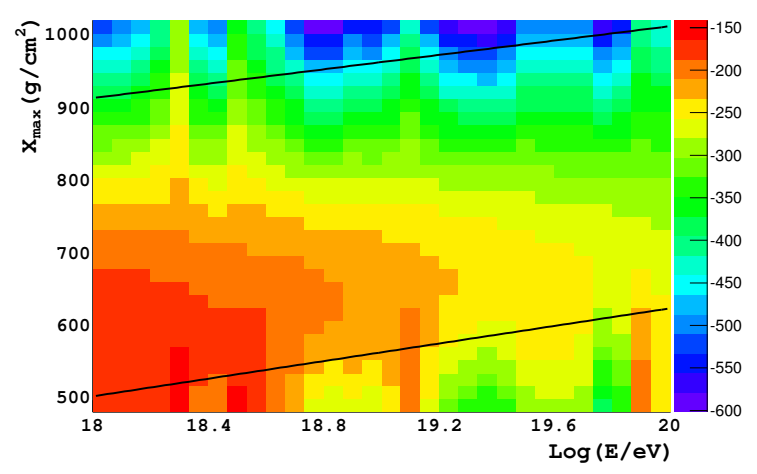

(a)

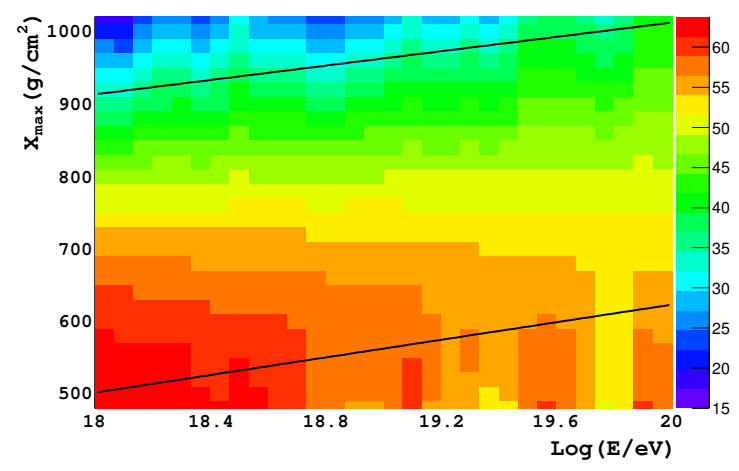

(c)

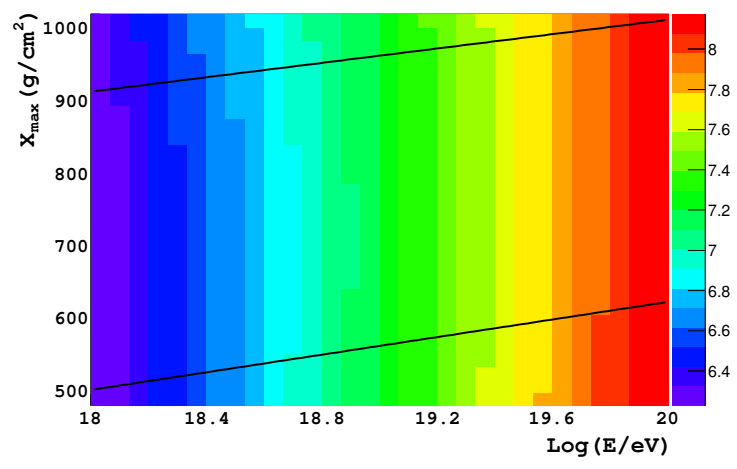

(e)

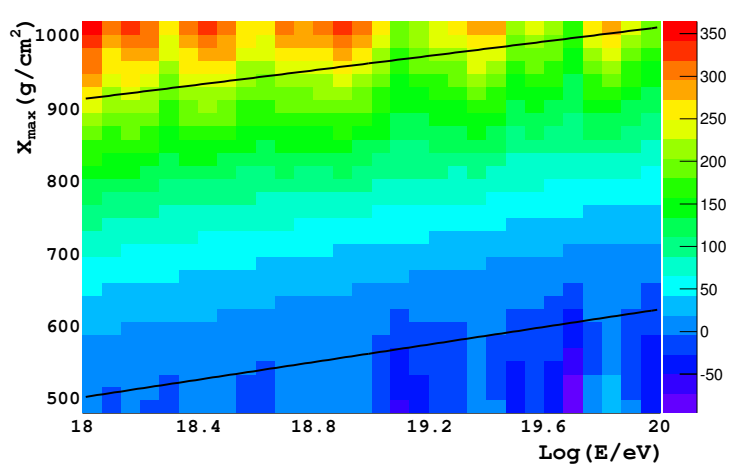

(b)

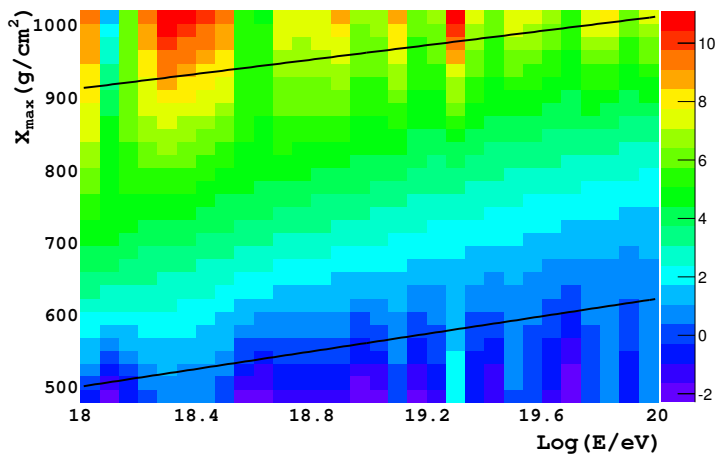

(d)

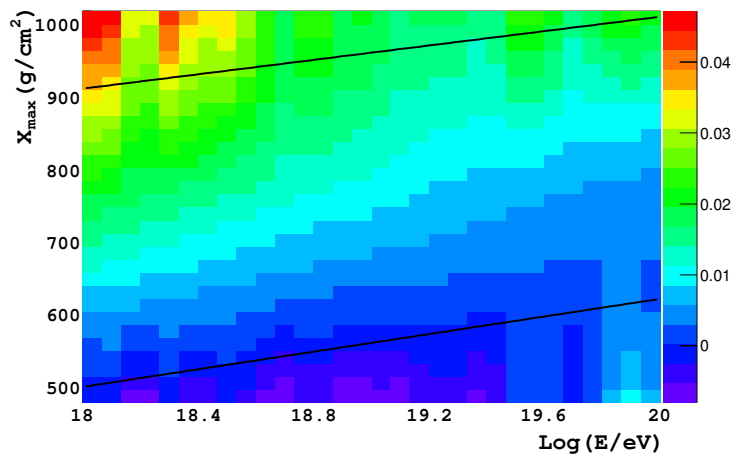

(f)

Figura 4.21 - Parametrização final de (a) $\mu_{X_{0}}$, (b) $\sigma_{X_{0}}$, (c) $\mu_{\lambda}$, (d) $\sigma_{\lambda}$, (e) $\mu_{\log \left(N_{\max }\right)}$ e (f) $\sigma_{\log \left(N_{\max }\right)}$ em função da energia (eixo $x$ ) $X_{\max }$ (eixo $y$ ). Os códigos das cores representam os parâmetros $\sigma^{\prime} s$ e $\mu^{\prime} s$. As linhas pretas definem os limites de utilização da parametrização. Fonte: Imagens elaboradas pelo autor. 


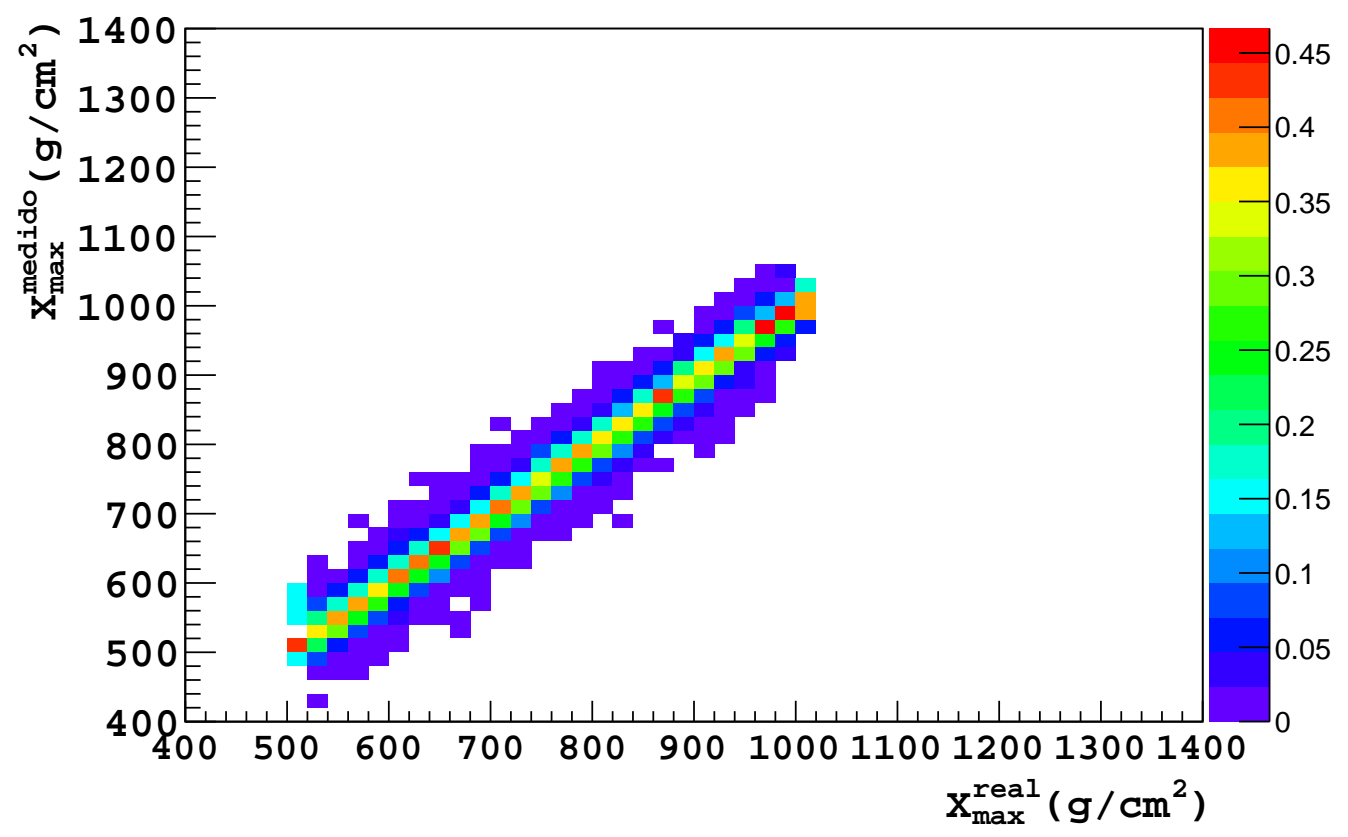

Figura 4.22 - Matriz de resposta do detector para o intervalo de energia definido por $18.4<$ $\log (E / e V)<18.5$. Fonte: Imagem elaborada pelo autor.

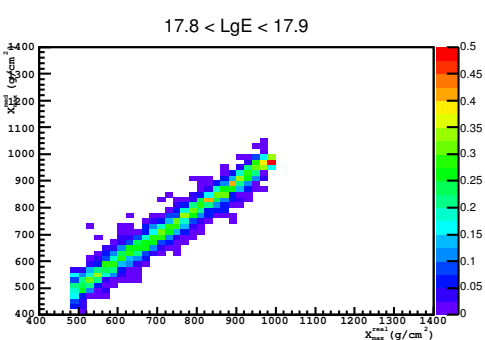

$18.1<\operatorname{Lg} \mathrm{E}<18.2$

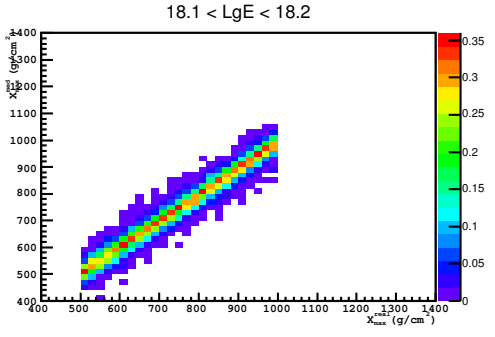

$18.4<\mathrm{LgE}<18.5$

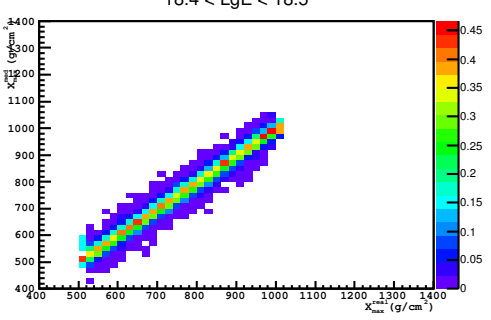

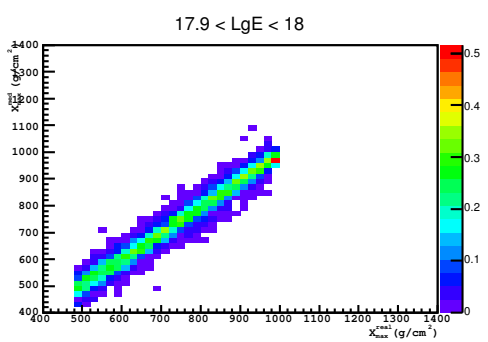

$18.2<\operatorname{LgE}<18.3$

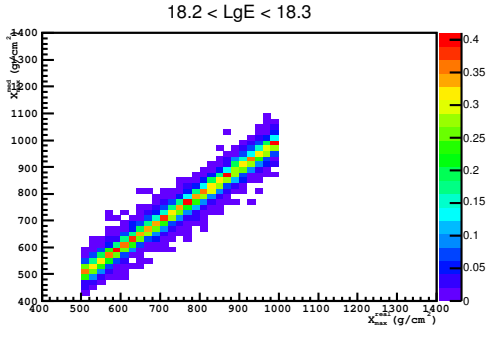

$18.5<\operatorname{LgE}<18.6$

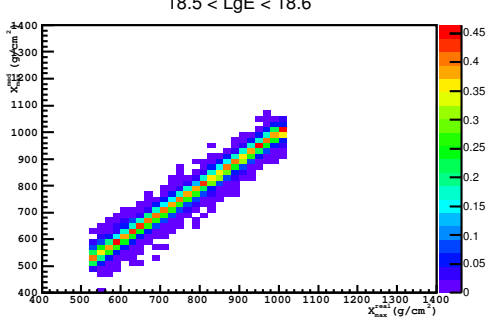

$18<\operatorname{LgE}<18.1$

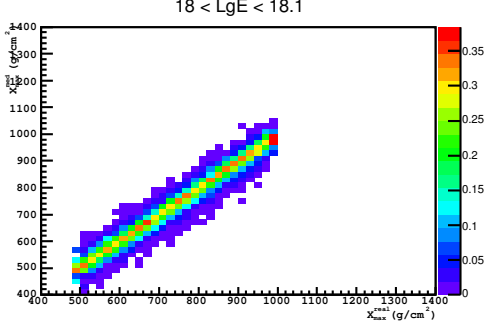

$18.3<\operatorname{LgE}<18.4$

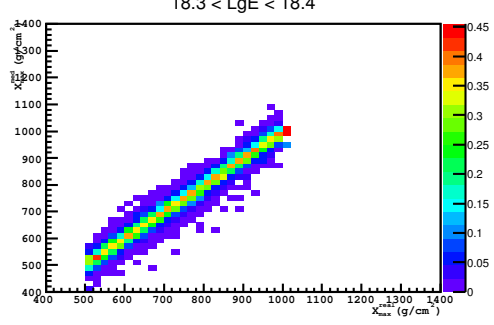

$18.6<\operatorname{LgE}<18.7$

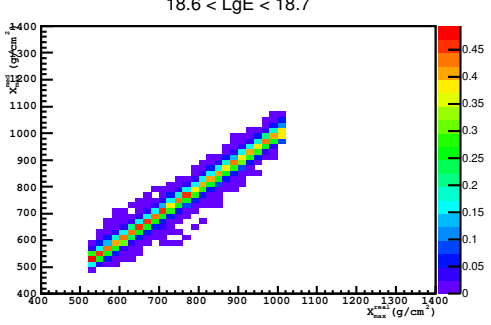

Figura 4.23 - Matrizes resposta do detector para os nove primeiros intervalos de energia. Fonte: Imagem elaborada pelo autor. 


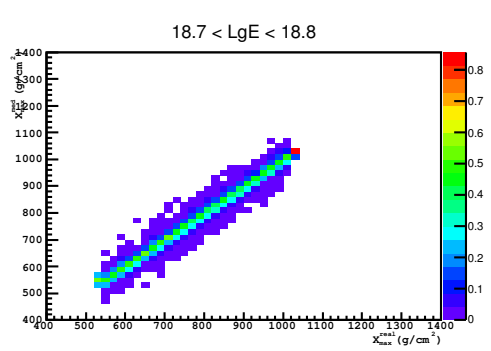

$19<\operatorname{LgE}<19.1$

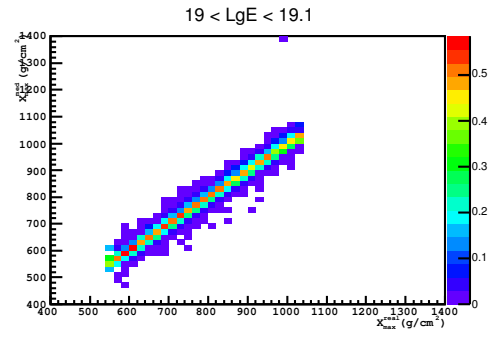

$19.3<\operatorname{LgE}<19.4$

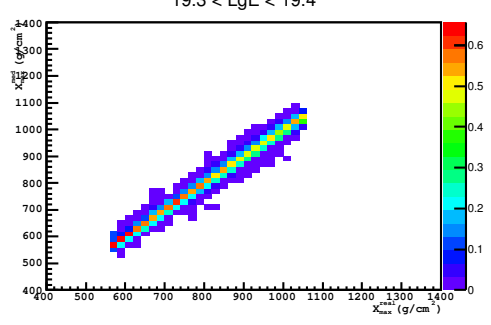

$18.8<\operatorname{LgE}<18.9$

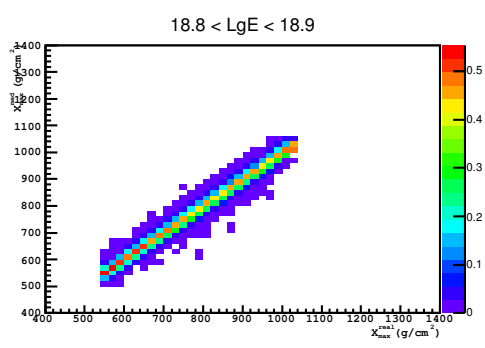

$19.1<\operatorname{LgE}<19.2$

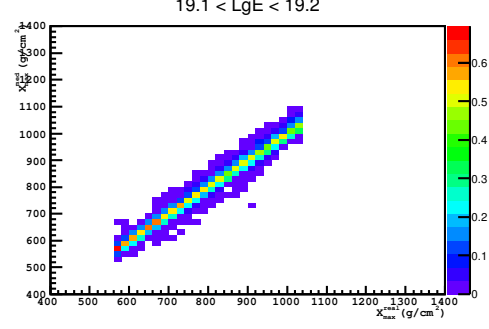

$19.4<\operatorname{LgE}<19.5$

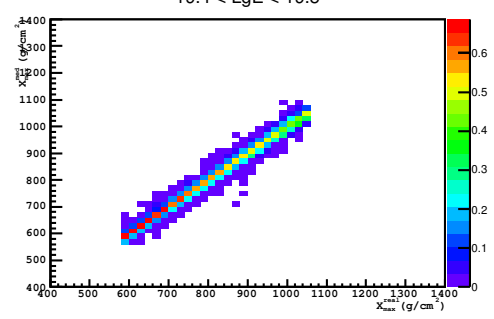

$18.9<\mathrm{LgE}<19$

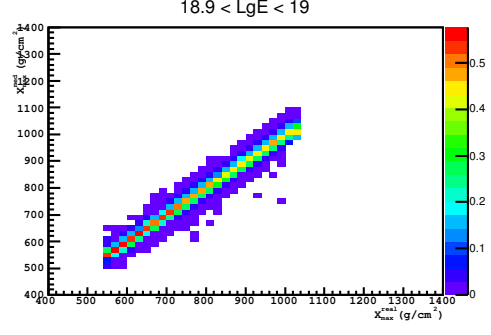

$19.2<\operatorname{LgE}<19.3$

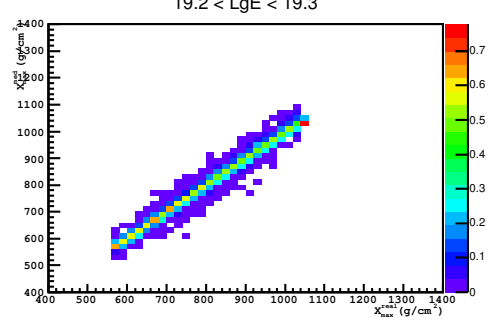

$19.5<\operatorname{LgE}<20$

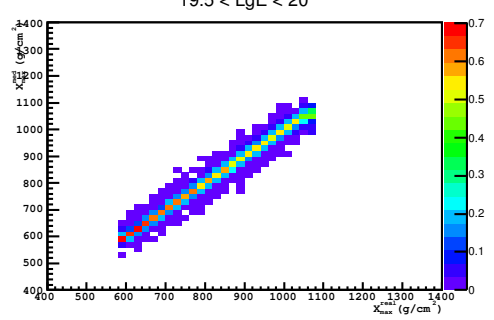

Figura 4.24 - Matrizes resposta do detector para os nove últimos intervalos de energia. Fonte: Imagem elaborada pelo autor. 

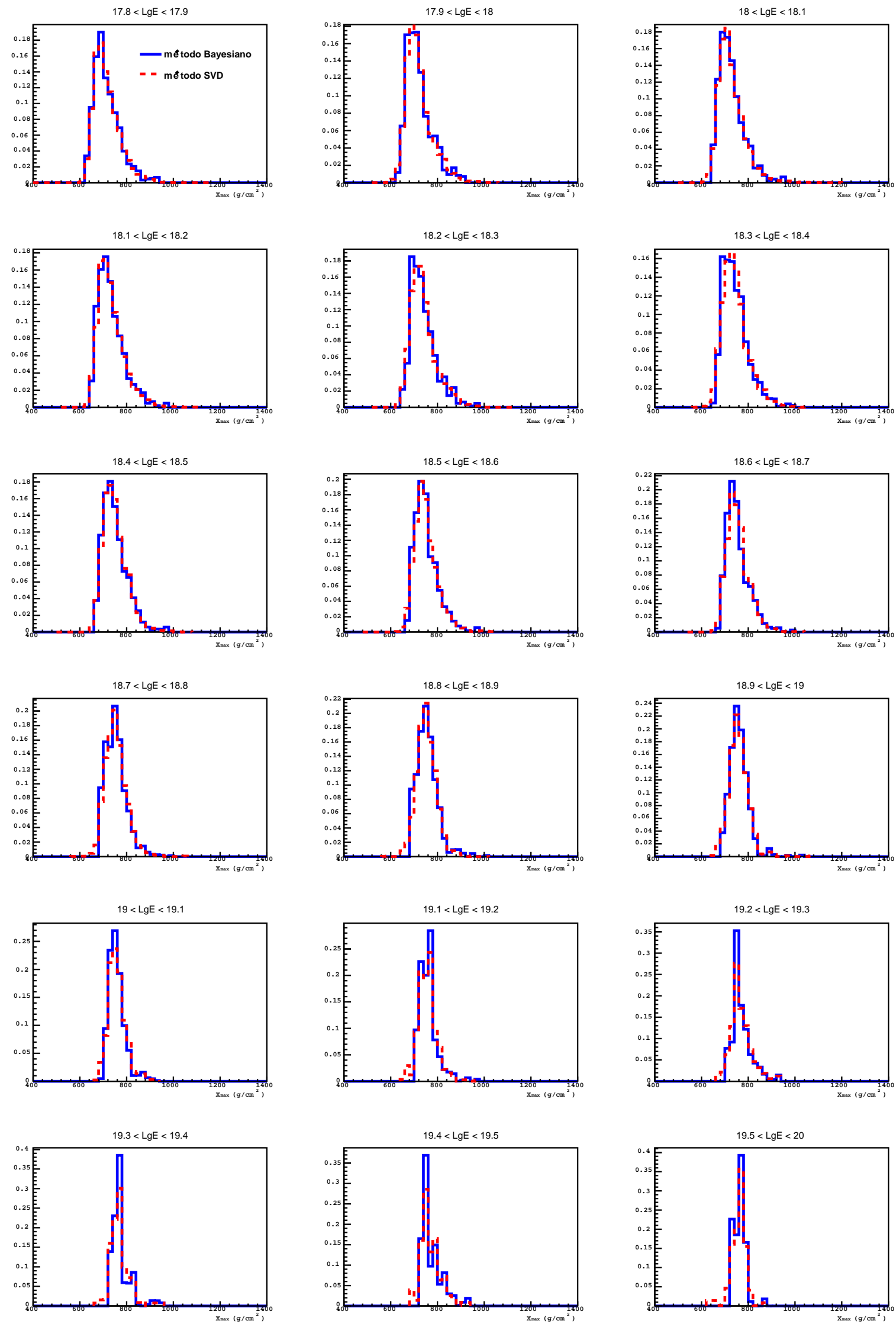

Figura 4.25 - Distribuições de $X_{\max }$ deconvoluídas para os dois métodos de deconvolução, SVD e bayesiano. Cada histograma representa um bin de energia. Fonte: Imagem elaborada pelo autor. 


\section{Análise multiparamétrica: redes neurais}

\subsection{Introdução}

\subsubsection{Análises multiparamétricas}

Ao longo das últimas décadas tem se tornado muito comum a aplicação de métodos estatísticos multiparamétricos em análises de dados de experimentos de física de altas energias (102). A maior motivação para a inclusão dessas novas ferramentas de análise é a possibilidade de extrair de maneira mais eficiente a informação contida nos dados e consequentemente obter resultados físicos mais precisos.

Alguns trabalhos já foram desenvolvidos com o objetivo de aplicar métodos multiparamétricos ao estudo de composição de raios cósmicos de altas energias, como pode ser visto nas referências (103-105). Em muitos desses trabalhos anteriores, é comum o fato dos métodos multiparamétricos serem aplicados apenas a eventos gerados por simulações, e não aos dados reais medidos por experimentos. Isso significa que, nesses casos, as complicações experimentais na determinação dos parâmetros sensíveis à composição não estão sendo levadas em conta, limitando muito a aplicabilidade das análises desenvolvidas. Incorporar os efeitos experimentais nas análises multiparamétricas é um dos problemas abordados pelo presente trabalho.

Os principais métodos multiparamétricos utilizados no contexto de física de altas energias são fortemente dependentes de simulações computacionais, e portanto, a confiança nos modelos teóricos é muito importante. Nesse sentido surge outra grande dificuldade na aplicação de tais ferramentas ao estudo de raios cósmicos de altas energias: a incerteza na descrição dos chuveiros atmosféricos através de modelos teóricos. Atualmente existe grande segurança por parte da comunidade de astrofísica de partículas a cerca da descrição teórico-computacional do desenvolvimento da componente eletromagnética dos chuveiros atmosféricos. Entretanto, 
o mesmo não é verdade com relação as componentes muônica e hadrônica. Existem evidências indicando que o número de múons contidos nos chuveiros medidos pelo Observatório Pierre Auger é maior que o número de múons existentes nos chuveiros gerados pelas simulações (106-109). Uma vez que as medidas dos telescópios de fluorescência dependem quase completamente da componente eletromagnética dos chuveiros e as medidas dos detectores de superfície são muito dependentes da componente muônica, escolhemos limitar esse trabalho à análise de parâmetros medidos apenas pelos telescópios de fluorescência do Observatório Pierre Auger e não incluir parâmetros medidos pelos detectores de superfície.

Entre os muitos métodos multiparamétricos existentes na literatura, as redes neurais (6) estão entre os mais utilizados como método de regressão não-linear. Alguns grupos já aplicam redes neurais a dados de experimentos de astrofísica de partículas, entre eles estão o IceCube (110, 111) e o PAMELA (112, 113). Neste trabalho utilizaremos redes neurais, mais precisamente uma classe chamada de Multilayer Perceptrons, para inferir a massa e a energia de astropartículas através de parâmetros extraídos dos perfis longitudinais medidos pelos telescópios de fluorescência do Observatório Pierre Auger.

\subsubsection{Redes neurais}

O funcionamento do cérebro humano, apesar de ainda pouco conhecido pela ciência atualmente, serviu de base para o desenvolvimento das chamadas redes neurais artificiais. De modo geral, o cérebro é formado por células chamadas neurônios, que se comunicam através da troca de sinais elétricos, as sinapses. A partir desse processo, os mais de $10^{11}$ neurônios contidos num cérebro humano são capazes de processar toda a informação necessária para executar as suas funções fisiológicas. Em analogia a esse sistema biológico tão eficiente, as redes neurais artificiais foram pensadas com o objetivo de ser uma ferramenta matemático-computacional capaz de processar informações, podendo ser usadas para abordar problemas como reconhecimento de padrões, inferência estatística, entre outros. A propriedade desses sistemas neurais biológicos de alterar a configuração das intensidades das interconexões entre os neurônios em resposta a estímulos externos é fundamental para que exista o processo de aprendizagem e por esse motivo essa propriedade foi transportada para as redes neurais artificiais.

A estrutura básica de uma rede neural artificial é baseada em modelos simplificados de neurônios, formada basicamente por nós e suas conexões. Matematicamente cada nó é caracterizado por uma função de ativação $g(x)$ e cada conexão entre nós é caracterizada por um valor $w_{i j}$ chamado de peso. Então, sendo $x_{i}$ variáveis atribuídas a cada nó, a resposta de um 


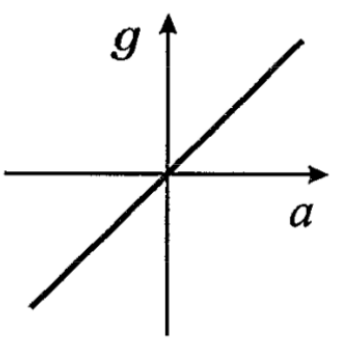

(a)

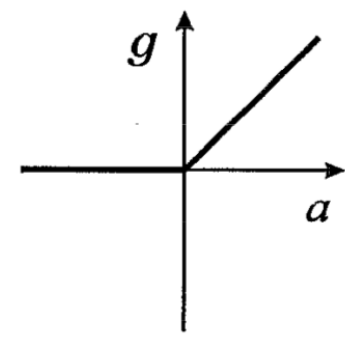

(c)

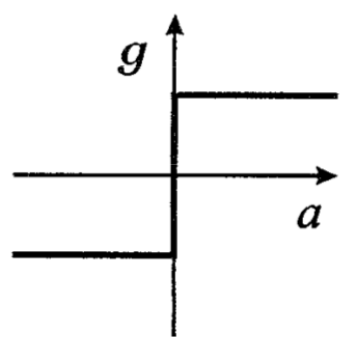

(b)

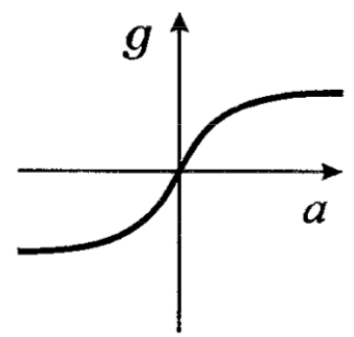

(d)

Figura 5.1 - Funções de ativação dos tipos (a) linear, (b) threshold, (b) threshold linear e (d) sigmoid. Fonte: Imagem extraída da referência (6).

dado nó é dada por

$$
y_{j}=g\left(\sum_{i} w_{i j} x_{i}\right) .
$$

As funções de ativação podem ser de vários tipos. A figura 5.1 ilustra os principais tipos de funções de ativação.

Esse trabalho será focado em um tipo especial de redes neurais chamado Multilayer Perceptron, que é muito utilizado em problemas de regressão não-linear. Nesse caso, os nós são divididos em camadas e cada nó de uma camada só se conecta com os nós das camadas adjacentes. A primeira camada contém os parâmetros de entrada e a última contém os parâmetros de saída. As outras camadas são chamadas de camadas intermediárias.

A ideia geral do processo de regressão através de Multilayer Perceptrons é procurar a melhor configuração dos pesos entre os nós para que, a partir dos parâmetros de entrada disponíveis, seja possível obter os parâmetros de saída com a maior precisão possível. A determinação da configuração ideal das conexões é feita utilizando um conjunto de dados em que os parâmetros de entrada e saída são conhecidos e esse processo é chamado de treinamento. Para cada problema abordado através de Multilayer Perceptrons deve-se definir o número de camadas e nós e o algoritmo de treinamento para otimizar a qualidade dos resultados. Os detalhes sobre a configuração e o processo de treinamento da rede neural usada nesse trabalho serão apresentados na seção 5.6. Na referência (6) pode ser encontrada uma descrição mais detalhada sobre redes neurais artificiais, Multilayer Perceptrons e suas aplicações. 


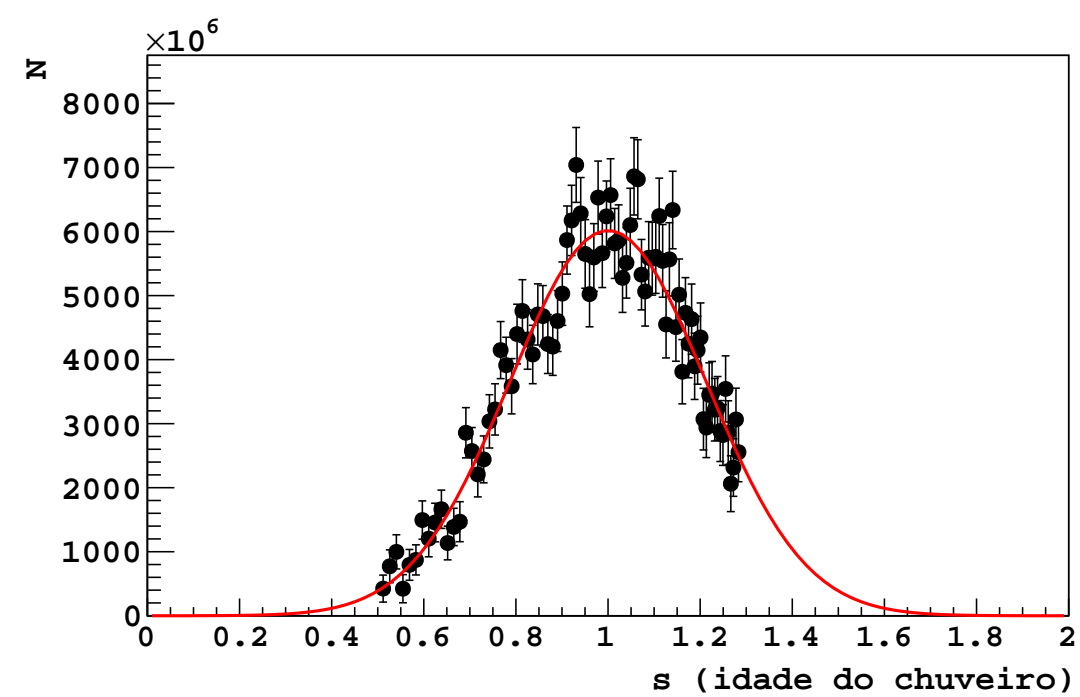

Figura 5.2 - Exemplo de um perfil longitudinal medido pelo Observatório Pierre Auger e ajustado pela função gaussiana na idade do chuveiro. $N$ é o número de partículas e $s$ é a idade do chuveiro. Os pontos pretos são os valores medidos e a curva vermelha é a função ajustada. Fonte: Imagem elaborada pelo autor.

\subsection{Definição dos parâmetros}

Na seção 3.3.3 mostramos como é feito a reconstrução do perfil longitudinal dos chuveiros medidos pelos telescópios de fluorescência do Observatório Pierre Auger. Além da função Gaisser-Hillas, outras funções podem ser utilizadas para ajustar o perfil medido. Entre elas, a gaussiana na idade do chuveiro (114) é bem conhecida e muito usada pela colaboração do HiRes (54). A idade do chuveiro é definida como

$$
s=\frac{3 X}{X+2 X_{\max }}
$$

em que $X$ é a profundidade atmosférica e $X_{\max }$ é a profundidade atmosférica na qual o chuveiro alcança o maior número de partículas. Logo, a função que descreve o perfil longitudinal através de uma gaussiana na idade do chuveiro é definida por

$$
N(s)=N_{\max } \cdot e^{-\frac{(s-1)^{2}}{2 \cdot \sigma^{2}}} .
$$

A função descrita pelas equações 5.2 e 5.3 possui apenas três parâmetros livres de ajuste $\left(N_{\max }, X_{\max }\right.$ e $\left.\sigma\right)$, diferentemente da função Gaisser-Hillas que possui quatro parâmetros livres $\left(N_{\max }, X_{\max }, X_{0}\right.$ e $\left.\lambda\right)$. A figura 5.2 mostra um exemplo de um perfil longitudinal medido pelo Observatório Pierre Auger e ajustado pela gaussiana na idade do chuveiro. 


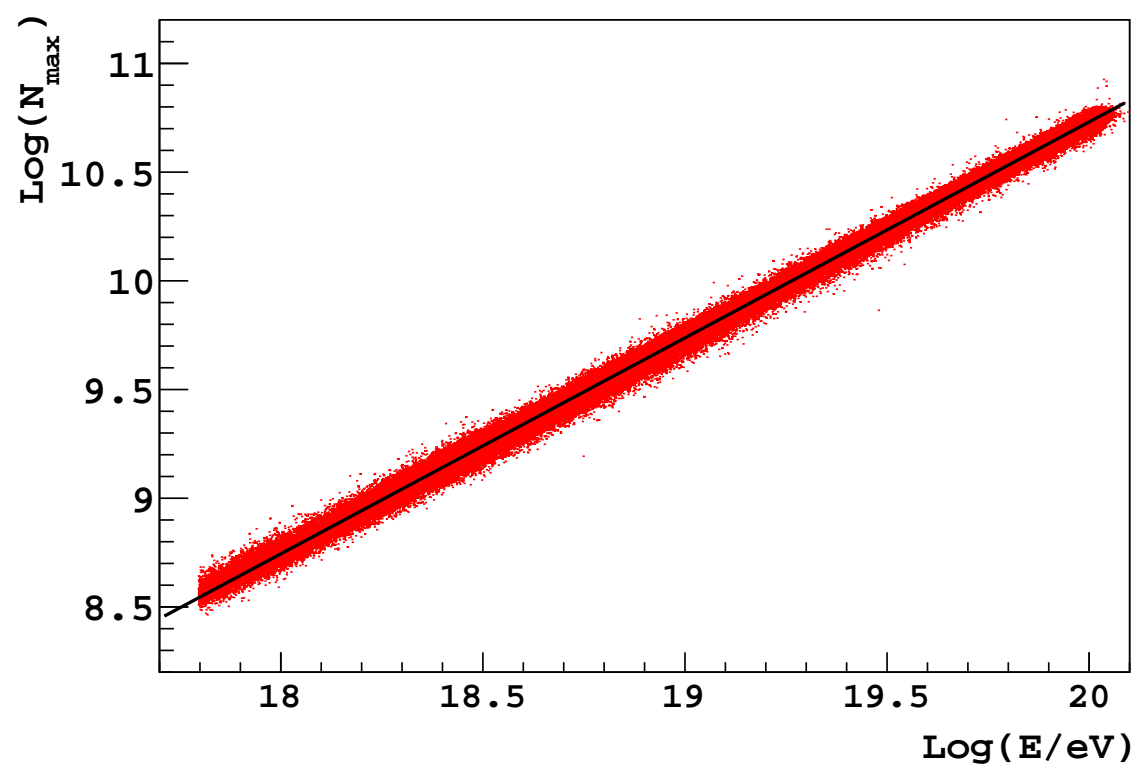

Figura 5.3 - Gráfico de $\log \left(N_{\max }\right) \times \log (E)$ mostrando a forte correlação do parâmetro $\log \left(N_{\max }\right)$ com a energia primária do chuveiro. Os dados foram extraídos de um conjunto de chuveiros simulados pelo software CONEX (30, 31) e reconstruídos pelo Offline (95). O modelo de interação hadrônica utilizado foi o QGSJetII-04 (99, 100). Fonte: Imagem elaborada pelo autor.

O fato do $X_{\max }$ carregar informação sobre a composição da partícula primária do chuveiro é bem conhecido e pode ser verificado pela análise apresentada no capítulo 4. Tal como o $X_{\max }$, o parâmetro $\sigma$ também carrega informação sobre a composição da partícula primária. A figura 5.14 mostra a evolução com a energia da média de $\sigma$ e podemos observar que os chuveiros simulados com diferentes tipos de primários tem valores significativamente diferentes de $\langle\sigma\rangle$.

O $N_{\text {max }}$ é um fator de normalização do perfil longitudinal e está fortemente correlacionado com a energia total do chuveiro. Essa correlação pode ser observada na figura 5.3, que mostra um gráfico de $\log \left(N_{\max }\right) \times \log (E)$ para um conjunto de chuveiros simulados. Como o objetivo dessa análise é inferir a massa e a energia da partícula primária através dos parâmetros extraídos do perfil longitudinal medido, escolhemos utilizar os três parâmetros definidos pela função dada na equação $5.3\left(X_{\max }, \sigma\right.$ e $\left.N_{\max }\right)$ como parâmetros de entrada para as redes neurais.

Como foi dito na seção 5.1.1, os efeitos dos detectores na determinação dos parâmetros do perfil longitudinal são muito relevantes para o tipo de análise que será desenvolvida nesse trabalho. Por esse motivo, incluiremos os efeitos experimentais da reconstrução nas simulações que serão utilizadas no treinamento da rede neural. Isso significa que os parâmetros $N_{\max }$, 
$X_{\text {max }}$ e $\sigma$ serão extraídos após os chuveiros simulados serem reconstruídos levando em conta o processo de medida híbrido do Observatório Pierre Auger.

A resolução na determinação dos parâmetros do perfil longitudinal é o principal efeito dos detectores nas medidas. A figura 5.4 mostra as distribuições das diferenças entre os valores reconstruídos e reais para os parâmetros $X_{\max }, \sigma$ e $\log \left(N_{\max }\right)$ obtidos através de simulações. O $R M S$ dessas distribuições pode ser usado como uma medida da resolução do detector. A figura 5.5 mostra a evolução com a energia da resolução para cada um dos parâmetros, calculadas através de simulações. É possível observar que o efeito experimental incluso pelo processo de medida é significativo, sendo da ordem de $\sim 20 \mathrm{~g} / \mathrm{cm}^{2}, \sim 0.02 \mathrm{e} \sim 0.03$ para o $X_{\max }, \sigma$ e $\log \left(N_{\max }\right)$ respectivamente.

Além dos parâmetros de entrada definidos acima, definiremos os dois parâmetros de saída das redes neurais, sendo um relativo a energia e o outro relativo a massa da partícula primária. O parâmetro relativo a energia será o logaritmo de seu valor, $\log (E / \mathrm{eV})$ e o parâmetro de saída relativo a massa será definido como $M L P=\ln (A) / \ln (56)$, onde $A$ é a massa nuclear da partícula primária. Essa definição pode ser interpretada como uma massa normalizada, que varia de 0 , para prótons, até 1 , para núcleos de ferro.

\subsection{Simulações}

Os chuveiros simulados utilizados nesse trabalho foram gerados pelo software CONEX (30, 31). A reconstrução foi feita pelo software Offline (95), desenvolvido pela colaboração do Observatório Pierre Auger. Nesse processo foram consideradas as condições reais de trabalho dos detectores, incluindo condições técnicas e atmosféricas. O modelo de interação hadrônica utilizado foi o QGSJetll-04 (99, 100). Os tipos de primários selecionados foram núcleos de hidrogênio (prótons), hélio, nitrogênio, silício e ferro, com valor de massa nuclear de $A=1$, $A=4, A=14, A=28$ e $A=56$, respectivamente. A seleção dos eventos simulados foi feita seguindo os critérios descritos na seção 5.5 .

A figura 5.6 mostra o número de chuveiros simulados para cada primário, em cada intervalo de energia, que foram selecionados para essa análise. O número total de eventos simulados foi de 14598, 8747, 14782, 12020 e 15325 para núcleos de hidrogênio, hélio, nitrogênio, silício e ferro, respectivamente. 


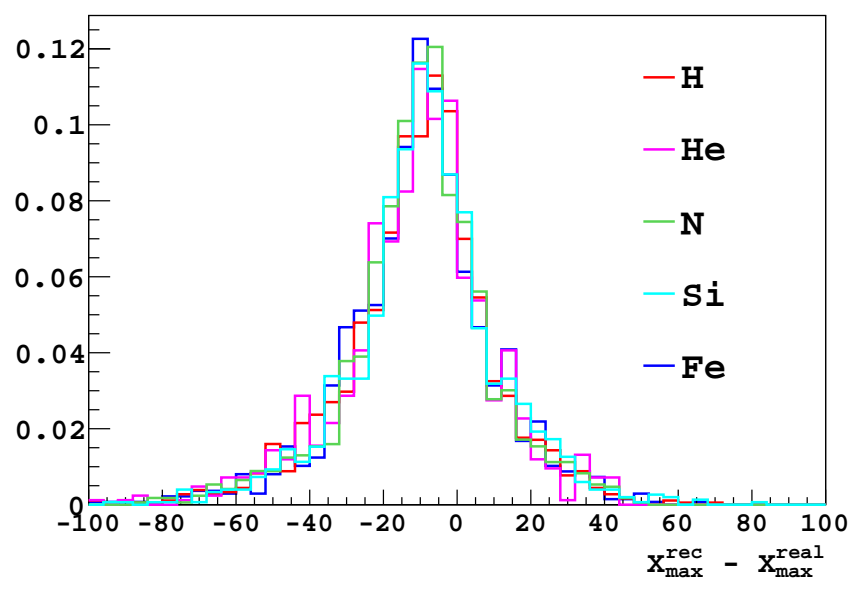

(a)

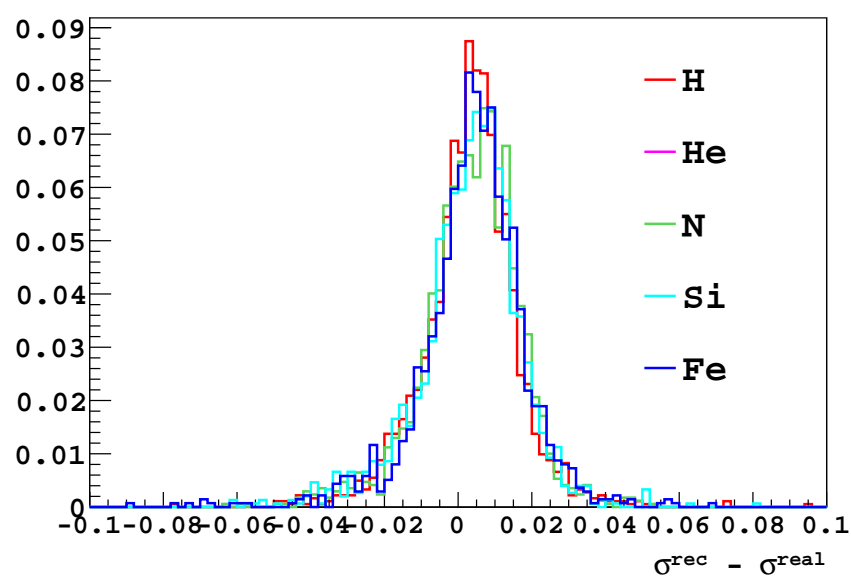

(b)

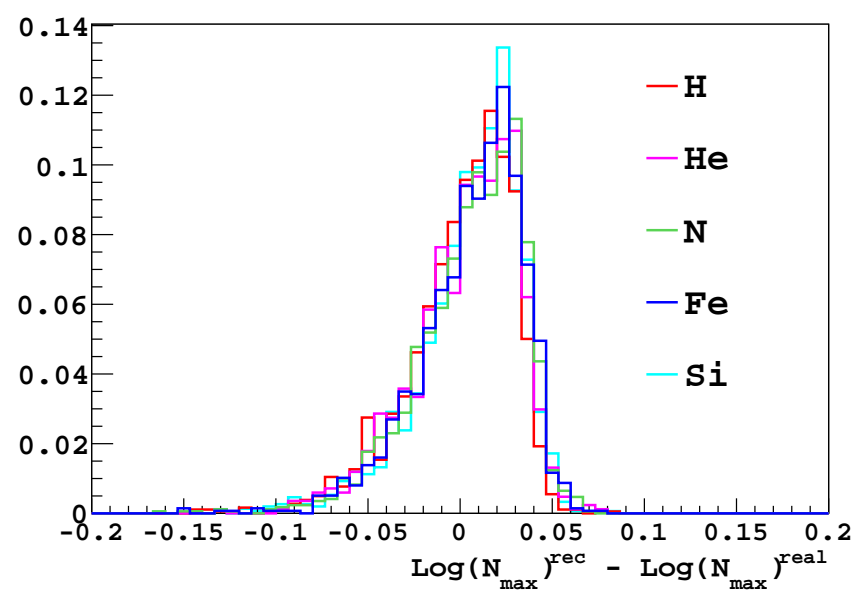

(c)

Figura 5.4 - Distribuições das diferenças entre os parâmetros reconstruídos e simulados, para (a) $X_{\max }$, (b) $\sigma$ e (c) $\log \left(N_{\max }\right)$. O intervalo de energia dos eventos selecionados para esse exemplo é $19.0<\log (E / \mathrm{eV})<19.2$. As distribuições estão normalizadas para facilitar a comparação entre diferentes primários. Fonte: Imagem elaborada pelo autor. 


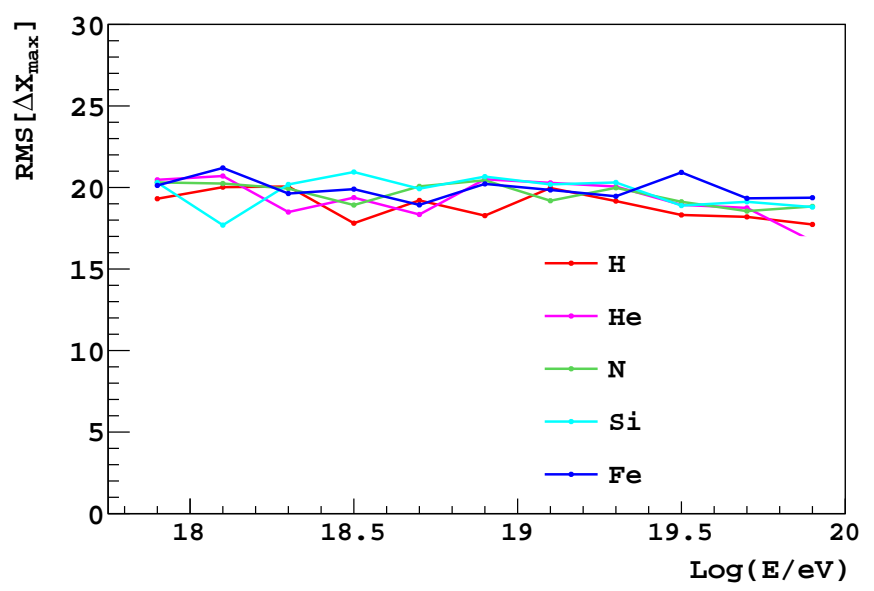

(a)

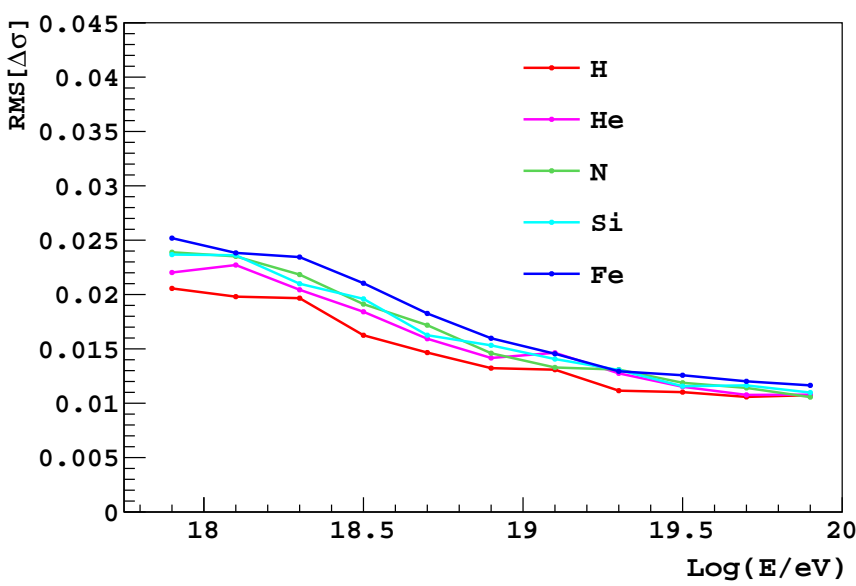

(b)

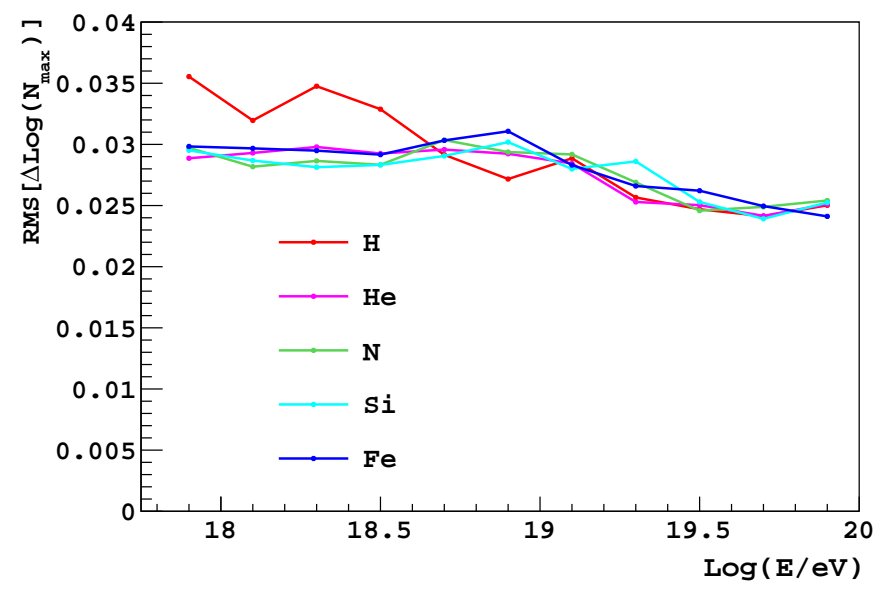

(c)

Figura 5.5 - Resolução dos parâmetros (a) $X_{\max }$, (b) $\sigma$ e (c) $\log \left(N_{\max }\right)$ em função da energia obtida através de chuveiros simulados. Fonte: Imagem elaborada pelo autor. 


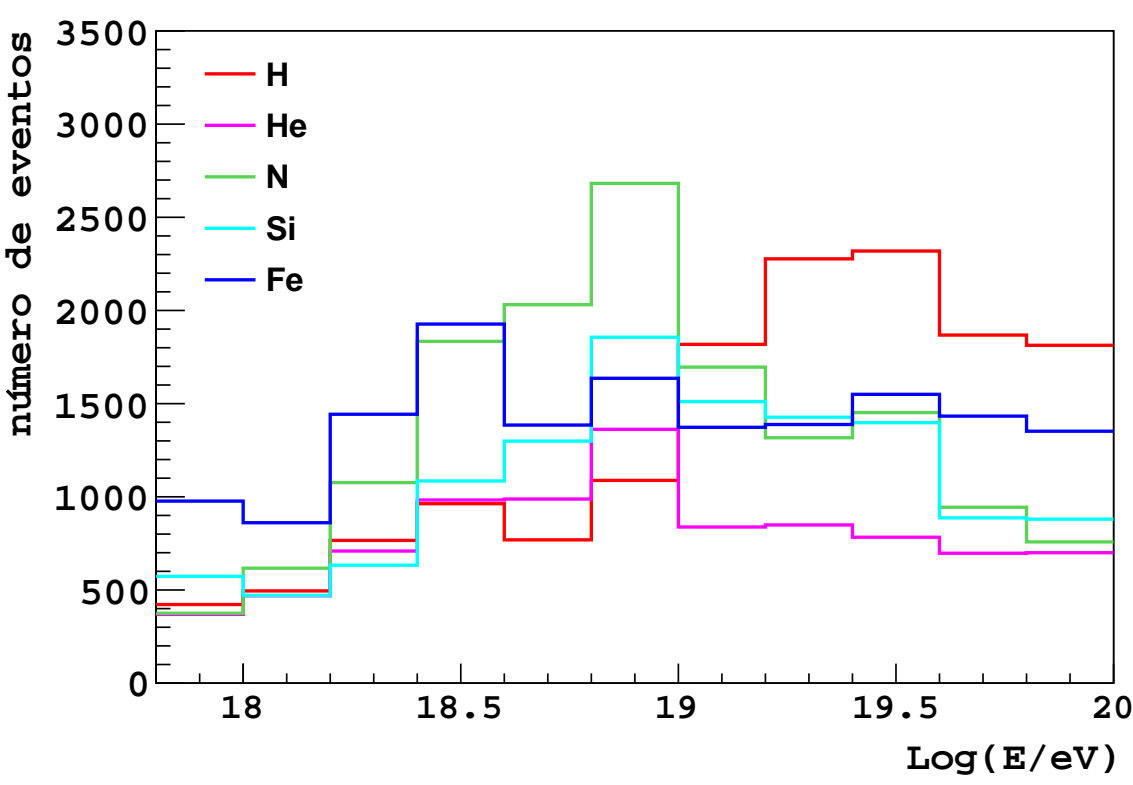

Figura 5.6 - Número de eventos simulados para cada tipo de primário por intervalo de energia. Fonte: Imagem elaborada pelo autor.

\subsection{Dados medidos}

Tal como na análise apresentada no capítulo 4, os dados utilizados nesse trabalho foram medidos de forma híbrida pelo Observatório Pierre Auger entre 04/12/2004 e 31/12/2012. $\mathrm{O}$ limite mínimo de energia para os eventos selecionados também foi de $10^{17.8} \mathrm{eV}$. Os bins de energia foram divididos em intervalos de $\Delta \log (E / \mathrm{eV})=0.2$ entre $10^{17.8} \mathrm{eV}$ e $10^{19.4} \mathrm{eV}$, e acima desse valor foi definido um único bin. Foram aplicados os mesmos cortes de qualidade básicos apresentados na seção 4.4.1. Além desse cortes, foram aplicados os critérios de seleção descritos na seção 5.5. A figura 5.7 mostra o número de eventos selecionado para essa análise em cada intervalo de energia.

\subsection{Seleção dos eventos}

A seleção dos chuveiros utilizados nessa análise pode ser dividida em duas etapas. A primeira delas envolve a aplicação dos mesmos cortes de qualidade descritos na seção 4.4.1. Esses cortes tem como objetivo garantir a qualidade mínima da reconstrução do perfil longitudinal.

A segunda etapa da seleção utiliza características geométricas do perfil longitudinal me- 


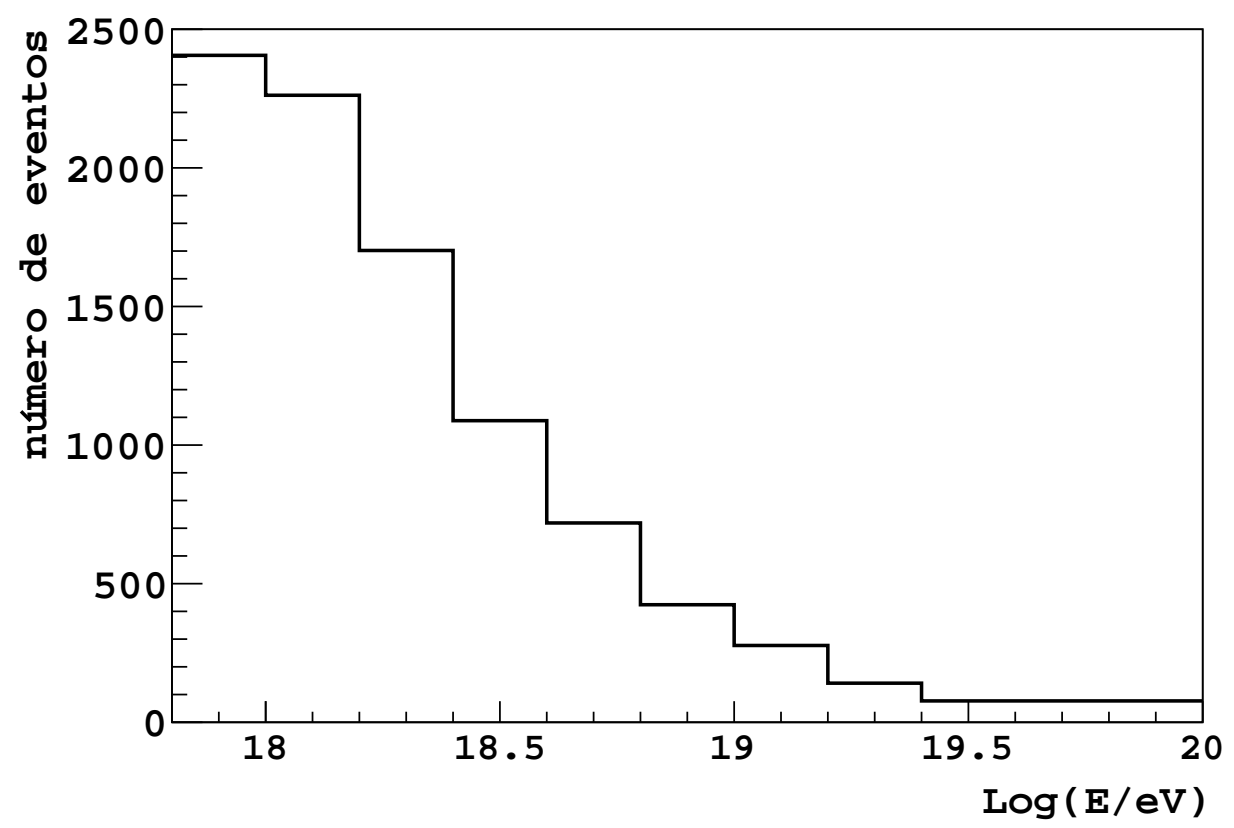

Figura 5.7 - Número de eventos selecionados para essa análise para cada bin de energia. Fonte: Imagem elaborada pelo autor.

dido para selecionar eventos em que os parâmetros $X_{\max }$ e $\sigma$ são reconstruídos com nível adequado de fidelidade. Nesse caso, os parâmetros geométricos que serão avaliados são as posições dos extremos do intervalo medido pelos telescópios de fluorescência. Em termos de profundidade atmosférica essas posições são chamadas de $X_{i n f}$ e $X_{\text {sup }}$, a posição inferior e superior respectivamente. Nesse trabalho iremos avaliar essas posições em termos da idade do chuveiro, o que, pela equação 5.2, significa $s_{i n f}=\frac{3 X_{i n f}}{X_{i n f}+2 X_{\max }}$ e $s_{\text {sup }}=\frac{3 X_{\text {sup }}}{X_{\text {sup }}+2 X_{\max }}$. Valores de $s_{\text {inf }}$ e $s_{u p}$ próximos de 1 significam que os limites do perfil medido estão muito próximos do máximo de partículas $X_{\max }$ e portanto o ajuste da função gaussiana pode ser prejudicado, de maneira a gerar um viés nos valores de $X_{\max }$ e $\sigma$. As figuras 5.8 e 5.9 mostram a dependência de $X_{\max }$ e $\sigma$ com $s_{\text {inf }}$ e $s_{\text {sup }}$. Pode-se observar que $X_{\max }$ e $\sigma$ são aproximadamente constantes nas regiões que $s_{i n f}$ e $s_{\text {sup }}$ estão longes de 1 , porém esse comportamento não se mantém em regiões próximas a 1 . Nesse sentido os valores de $s_{\text {inf }}=0.8$ e $s_{\text {sup }}=1.2$ serão utilizados como valores limites de confiabilidade, o que significa que chuveiros com $s_{\text {inf }}<0.8$ e $s_{\text {sup }}>1.2$ serão descartados. 


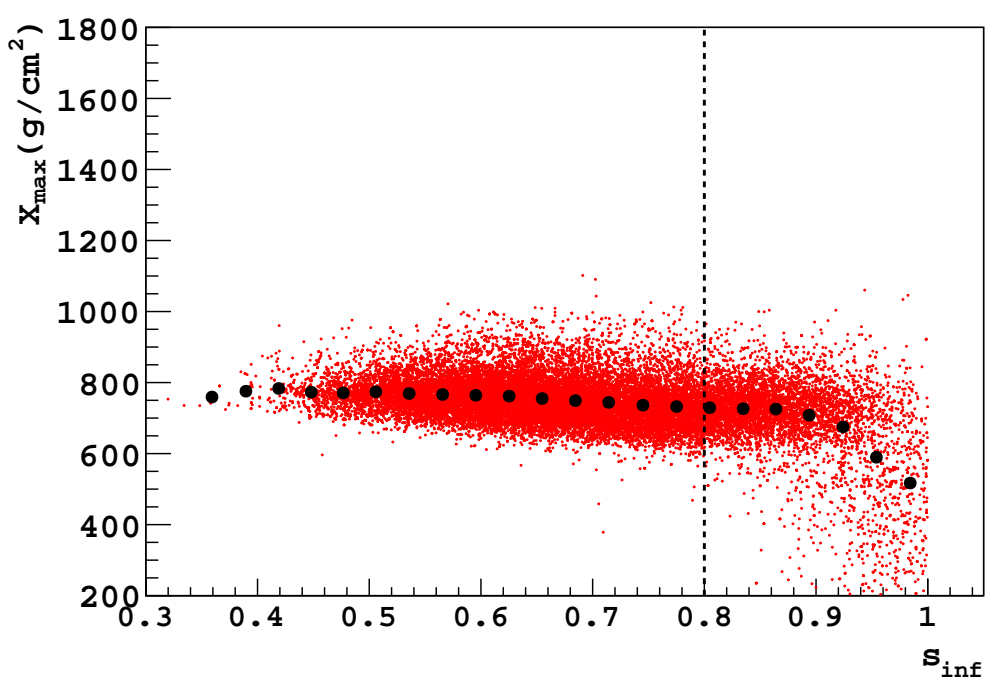

(a)

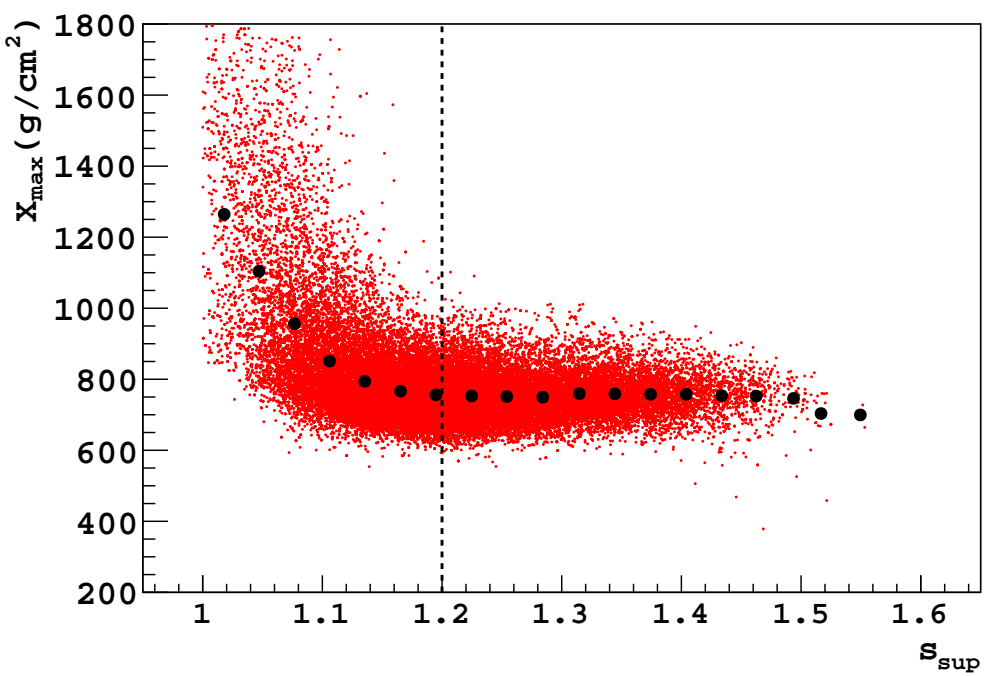

(b)

Figura 5.8 - Gráficos de (a) $X_{\max } \times s_{\text {inf }}$ e (b) $X_{\max } \times s_{\text {sup }}$. As linhas pretas mostram os limites $s_{\text {inf }}=0.8$ e $s_{\text {sup }}=1.2$ usados para separar os eventos enviesados e os não enviesados. Fonte: Imagem elaborada pelo autor. 


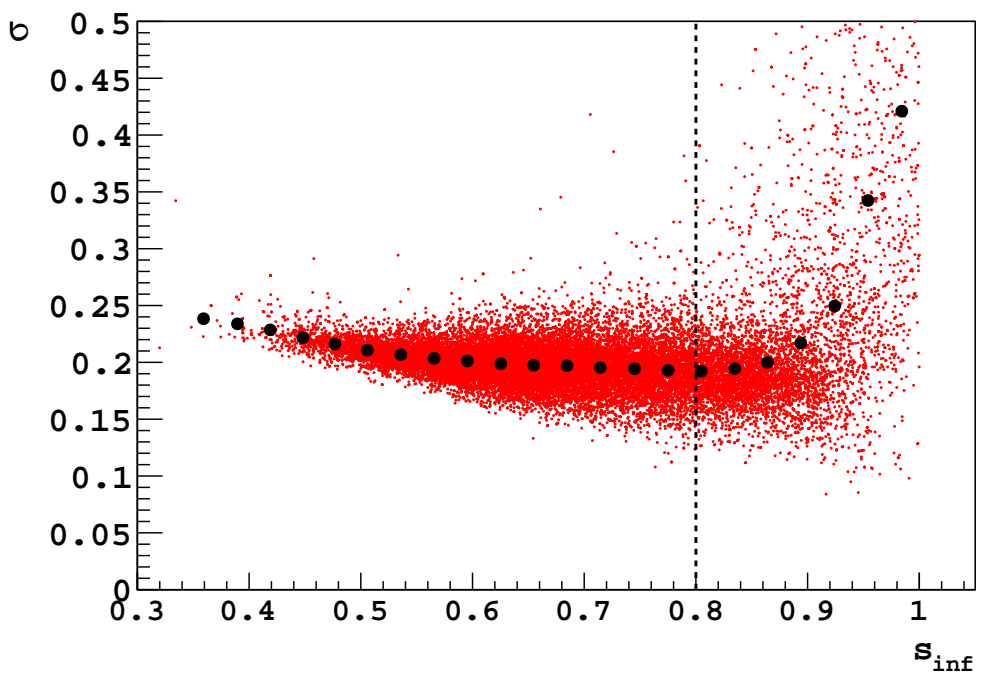

(a)

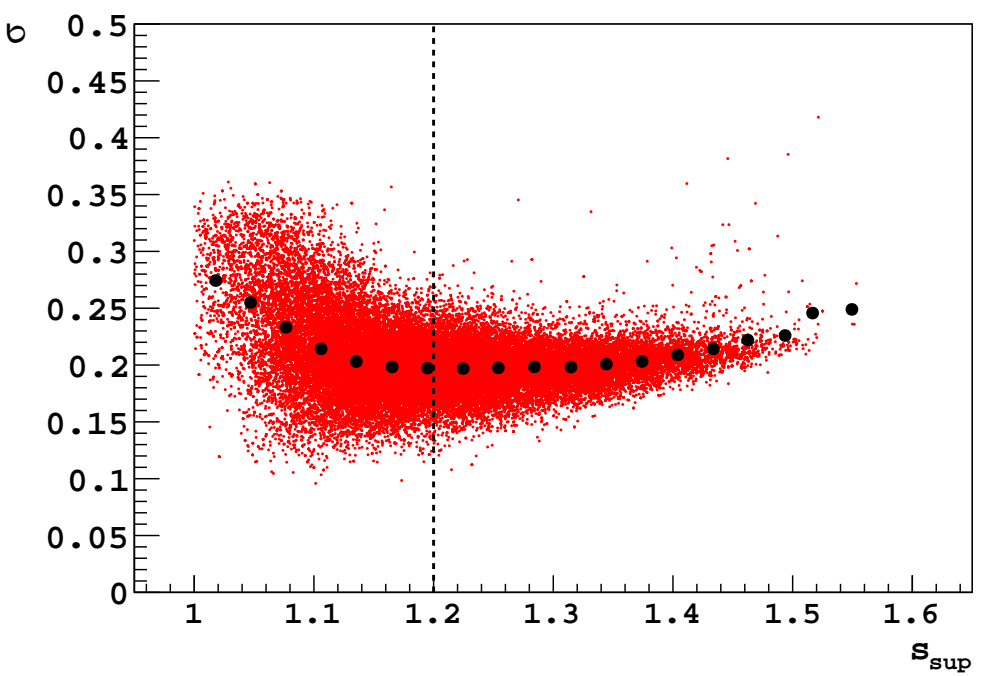

(b)

Figura 5.9 - Gráficos de (a) $\sigma \times s_{\text {inf }}$ e (b) $\sigma \times s_{\text {sup }}$. As linhas pretas mostram os limites $s_{\text {inf }}=0.8$ e $s_{\text {sup }}=1.2$ usados para separar os eventos enviesados e os não enviesados. Fonte: Imagem elaborada pelo autor. 


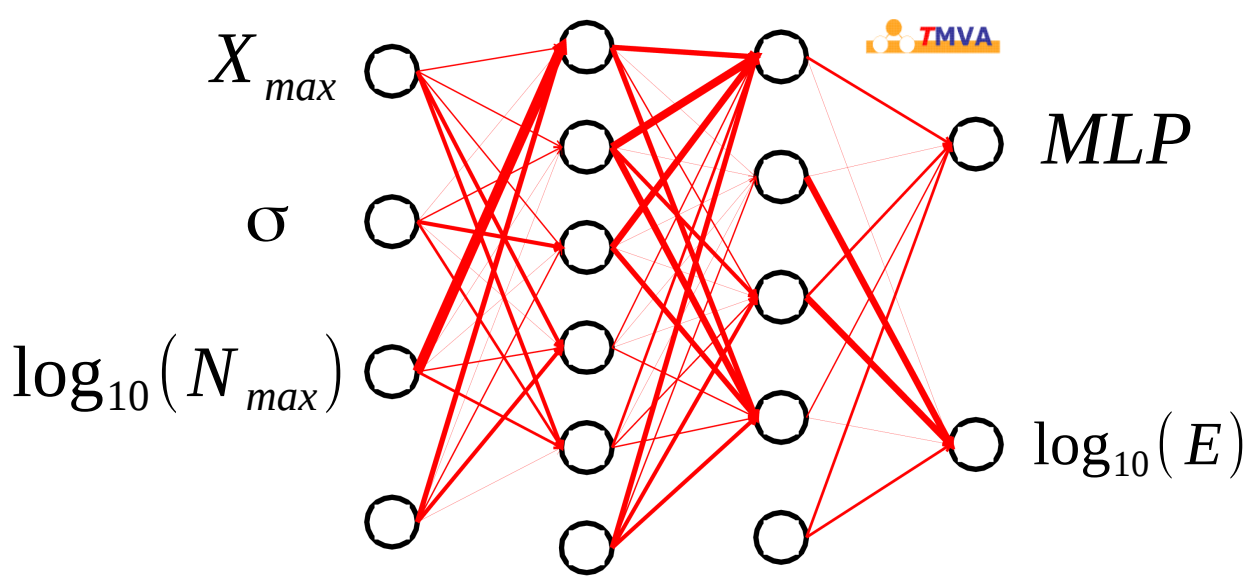

Figura 5.10 - Estrutura da rede neural usada por esse trabalho. Fonte: Imagem elaborada pelo autor.

\subsection{Configuração, treinamento e implementação da rede neural}

A configuração da rede neural escolhida foi do tipo 3:5:4:2, sendo três parâmetros de entrada, cinco e quatro nós nas camadas intermediárias e dois parâmetros de saída. Existem ainda um nó na camada de entrada e em cada uma das camadas intermediárias responsável por adicionar um termo offset. Alguns testes mostraram que essa configuração é suficiente para gerar os resultados mais precisos para nossa análise. Incluir mais camadas ou mais nós em cada camada seria redundante e não acrescentariam vantagens para o caso estudado nesse trabalho. A figura 5.10 mostra a configuração final da rede neural. A função de ativação escolhida para esse trabalho é a chamada função sigmóide, sugerida na referência (6).

Existem uma série de algoritmos destinados ao treinamento das redes neurais do tipo Multilayer Perceptrons. Para esse trabalho foi escolhido um método chamado Broyden-FletcherGoldfarb-Shanno (BFGS) (115-118). É possível encontrar na literatura resultados mostrando que esse algoritmo é o mais rápido (119). Os detalhes sobre o método não serão apresentados aqui, mas podem ser encontrados na referência (119).

Para o processo de treinamento, os chuveiros simulados descritos na seção 5.3 foram divididos aleatoriamente em duas amostras com o mesmo número de eventos: a amostra de treinamento e a amostra de testes. A amostra de treinamento é usada pelo algoritmo para determinar a melhor configuração da rede e a amostra de teste é usada para validar o processo. 
A implementação computacional das redes neurais desse trabalho foi feita através de uma ferramenta chamada Toolkit for Multivariate Analysis(TMVA) (119). O TMVA é uma biblioteca desenvolvida em ambiente ROOT (120) destinada à aplicação de métodos multiparamétricos e não é limitada apenas à redes neurais.

\subsection{Resultados e discussões}

Os resultados dessa análise serão apresentados em duas partes: a primeira referente a reconstrução da massa da partícula primária, na seção 5.7.1, e a segunda referente a reconstrução da energia, na seção 5.7.2.

\subsubsection{Massa nuclear}

Na seção 5.2 definimos o parâmetro $M L P$, que é obtido através das redes neurais e contém informação sobre a composição das partículas primárias dos chuveiros. Para avaliar a eficiência desse parâmetro quanto a separação de tipos de primários diferentes, utilizaremos uma grandeza chamada fator de mérito (FM), definido como

$$
F M=\frac{\bar{A}-\bar{B}}{\sqrt{\sigma_{A}^{2}+\sigma_{B}^{2}}},
$$

em que $\bar{A}$ e $\bar{B}$ são as médias e $\sigma_{A}$ e $\sigma_{B}$ são as variâncias das distribuições das grandezas $A$ e $B$. Calcularemos aqui o fator de mérito das distribuições do parâmetros obtidos por simulações de prótons e núcleos de ferro, que são os dois limites de tipos de primários considerados.

A figura 5.11 mostra os fatores de mérito calculados para os parâmetros $X_{\max }, \sigma, \log \left(N_{\max }\right)$ e $M L P$ em função da energia. Os eventos utilizados nessa etapa foram extraídos da amostra de testes. Notamos com relação aos parâmetros extraídos diretamente do perfil longitudinal que: a) o $X_{\max }$ contém a maior parte da informação sobre a composição tendo o maior fator de mérito, b) o $\sigma$ contém informação de composição significativa tendo fator de mérito em torno de 0.5 e c) o $\log \left(N_{\max }\right)$ praticamente não contém informação e portanto é pouco importante na análise de composição. Além disso, notamos que o parâmetro de saída das redes neurais $M L P$ tem fator de mérito maior que de qualquer parâmetro extraído do perfil, sendo mais eficiente como parâmetro destinado ao estudo da composição. Esse fato mostra que a análise por redes neurais, como apresentada nesse trabalho, é vantajosa em relação as análises 


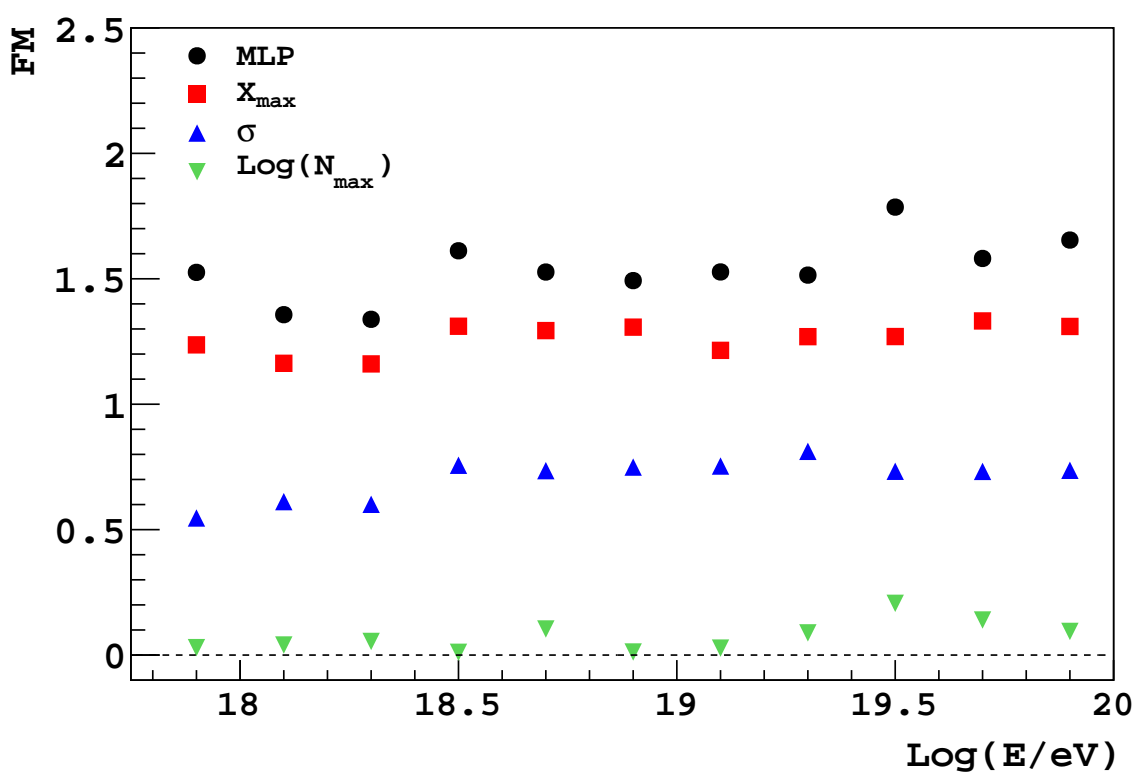

Figura 5.11 - Fator de mérito (FM) em função da energia para os parâmetros $X_{\max }, \sigma, \log \left(N_{\max }\right)$ e MLP. O FM foi calculado usando as distribuições dos parâmetros de chuveiros simulados iniciados por prótons e núcleos de ferro. Fonte: Imagem elaborada pelo autor.

monoparamétricas.

Para verificar a importância de cada parâmetro na eficiência da análise de composição apresentada nesse trabalho, executamos a mesma análise utilizando como parâmetros de entrada para as redes neurais apenas dois parâmetros entre $X_{\max }, \sigma$ e $\log \left(N_{\max }\right)$. Os fatores de mérito calculados para o parâmetro $M L P$ em função da energia para cada caso estão apresentados na figura 5.12. Novamente foram utilizados os eventos da amostra de testes. Observamos primeiramente que o fator de mérito no caso do par $\sigma+\log \left(N_{\max }\right)$ é o mais baixo, e portanto podemos concluir que o $X_{\max }$ é o parâmetro mais importante para nossa análise.

Comparando os resultados para os pares $X_{\max }+\sigma$ e $X_{\max }+\log \left(N_{\max }\right)$ vemos que: a) para energias abaixo de $\sim 10^{18.7} \mathrm{eV}$ o fator de mérito para o par $X_{\max }+\log \left(N_{\max }\right)$ é maior, mostrando que nessa região de energia o $\log \left(N_{\max }\right)$ é mais importante que o $\sigma, \mathrm{e}$ b) para energias acima de $\sim 10^{18.7} \mathrm{eV}$ o fator de mérito para o par $X_{\max }+\sigma$ é maior, mostrando que nessa região de energia o $\sigma$ é mais importante que o $\log \left(N_{\max }\right)$. Logo, podemos concluir que a maior eficiência da análise em todas as regiões de energia é obtido usando os três parâmetros conjuntamente como parâmetros de entrada.

As figuras 5.13, 5.14 e 5.15 mostram a evolução com a energia de $\left\langle X_{\max }\right\rangle,\langle\sigma\rangle$ e $\langle M L P\rangle$ 


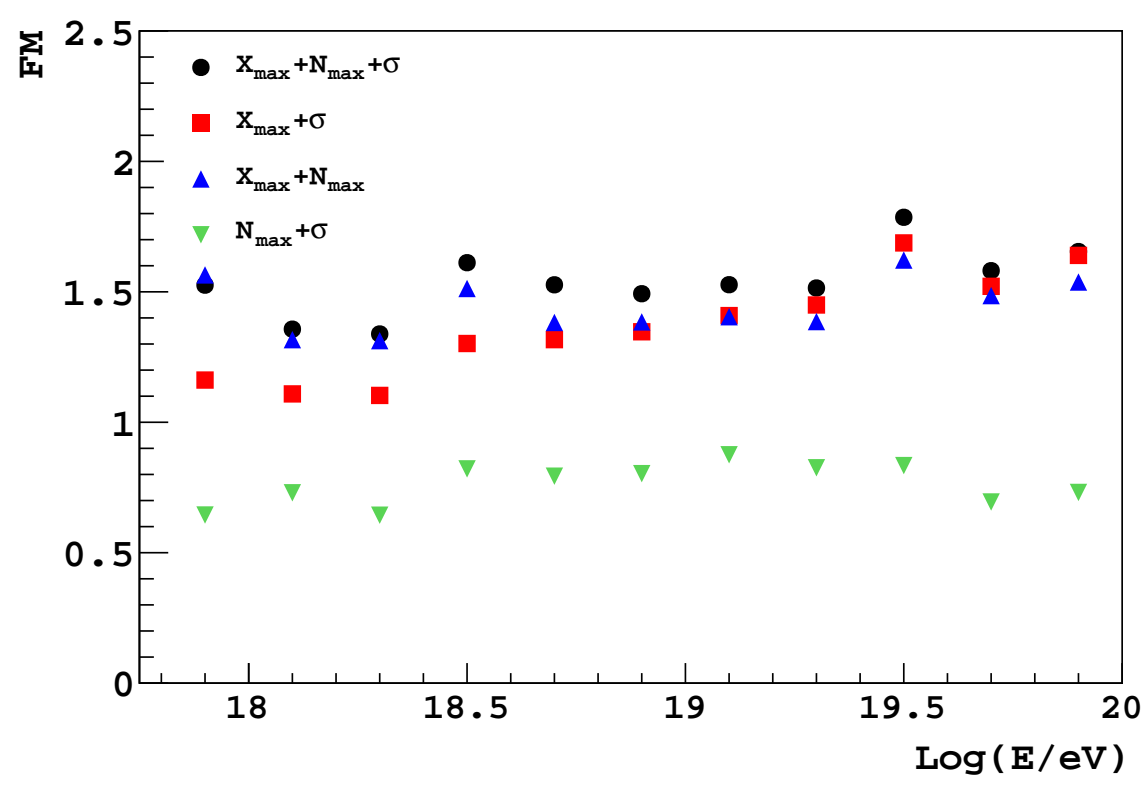

Figura 5.12 - Fator de mérito calculado para o parâmetro $M L P$ considerando os três parâmetros de entrada $\left(X_{\max }, \sigma, \log \left(N_{\max }\right)\right)$ e pares desses. Fonte: Imagem elaborada pelo autor.

para os dados medidos pelo Observatório Pierre Auger comparados com as simulações de núcleos de hidrogênio(prótons) e ferro. Observamos em todos os casos que os dados medidos estão em média muito próximos das simulações iniciadas por prótons. Para o caso do parâmetro $M L P$ (figura 5.15) podemos notar que com o aumento da energia os dados tendem a se afastar das simulações de próton. Esse tendência é mais clara no figura 5.16 que compara os dados às simulações de prótons e núcleos de hélio. Com o objetivo de avaliar com mais clareza o comportamento do parâmetro $M L P$, definimos o parâmetro $\alpha=\frac{\langle M L P\rangle_{\text {dado }}-\langle M L P\rangle_{H}}{\langle M L P\rangle_{H e}-\langle M L P\rangle_{H}}$, que mede basicamente a distância dos dados às simulações de próton e hélio. Na figura 5.17 mostramos a evolução de $\alpha$ com a energia. Observamos nesse caso que os dados tendem a se aproximar das simulações de próton até aproximadamente $10^{18.3} \mathrm{eV}$ e tendem a se afastar a partir dessa energia, mostrando uma mudança na composição média das partículas.

\subsubsection{Energia}

Para medir a eficiência da análise com relação a reconstrução da energia avaliaremos a resolução energética a partir dos chuveiros simulados que constituem a amostra de testes. A resolução é definida com $\left(E_{r e c}-E_{M C}\right) / E_{M C}$, em que $E_{r e c}$ é o valor da energia reconstruído pelas redes neurais ou pelo método padrão e $E_{M C}$ é o valor da energia real extraído das 


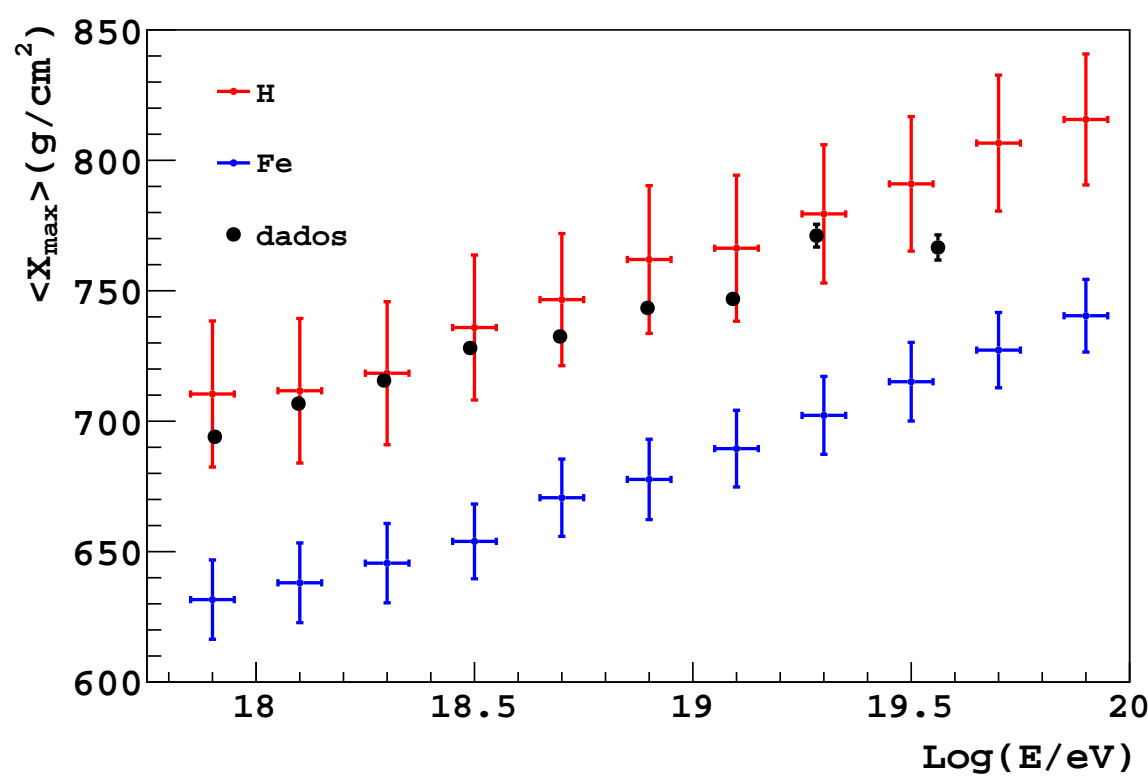

Figura 5.13 - Evolução do $X_{\max }$ com a energia para simulações de núcleos de hidrogênio e ferro e para os dados medidos pelo Observatório Pierre Auger. Fonte: Imagem elaborada pelo autor.

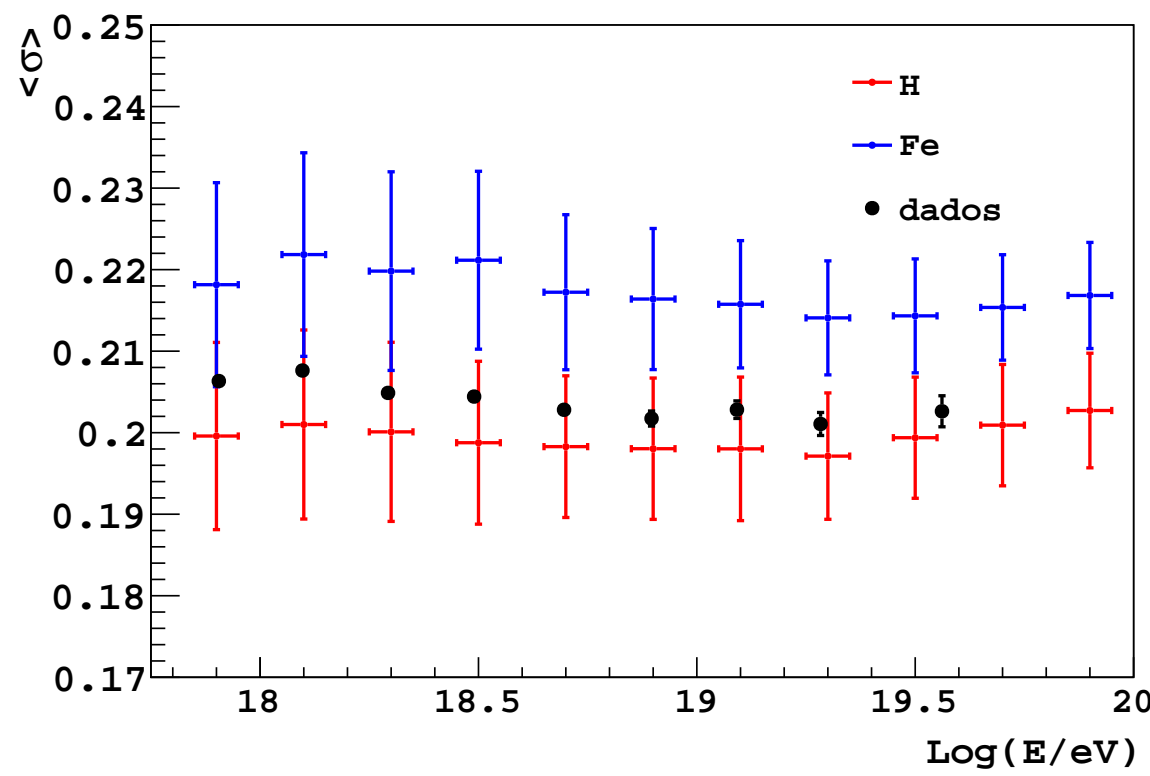

Figura 5.14 - Evolução do $\sigma$ com a energia para simulações de núcleos de hidrogênio e ferro e para os dados medidos pelo Observatório Pierre Auger. Fonte: Imagem elaborada pelo autor. 


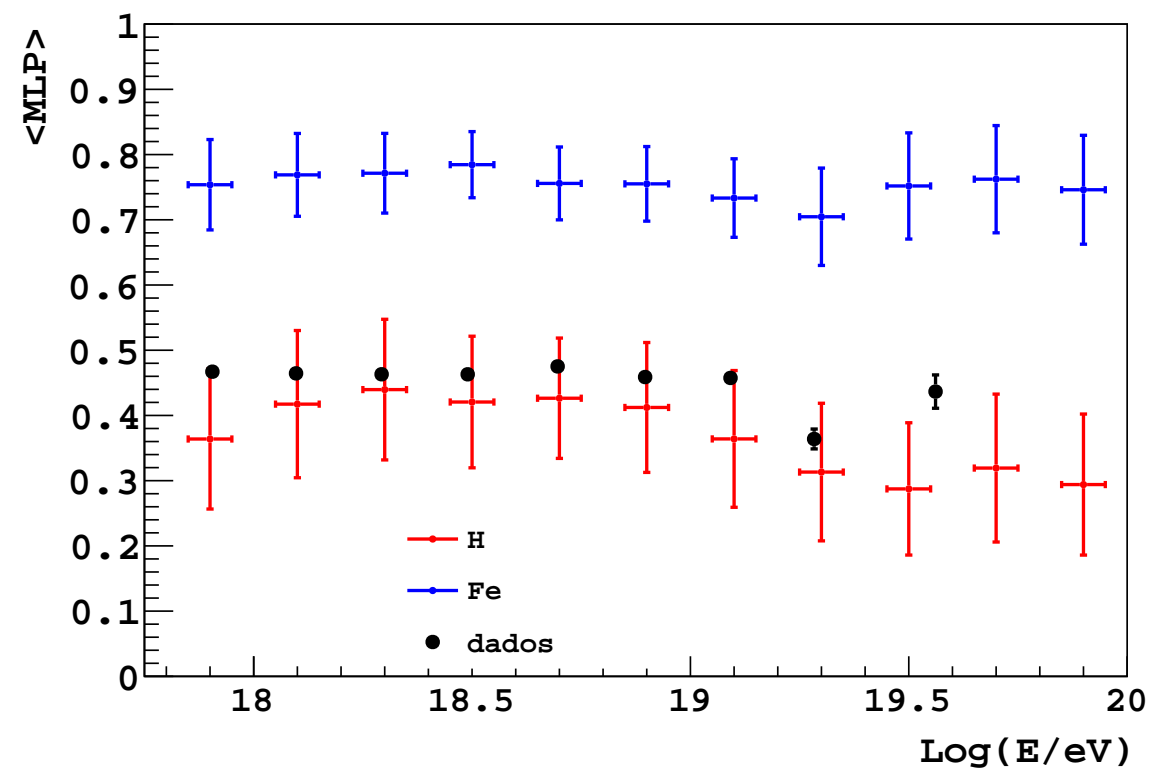

Figura 5.15 - Evolução do parâmetro $M L P$ com a energia para simulações de núcleos de hidrogênio e ferro e para os dados medidos pelo Observatório Pierre Auger. Fonte: Imagem elaborada pelo autor.

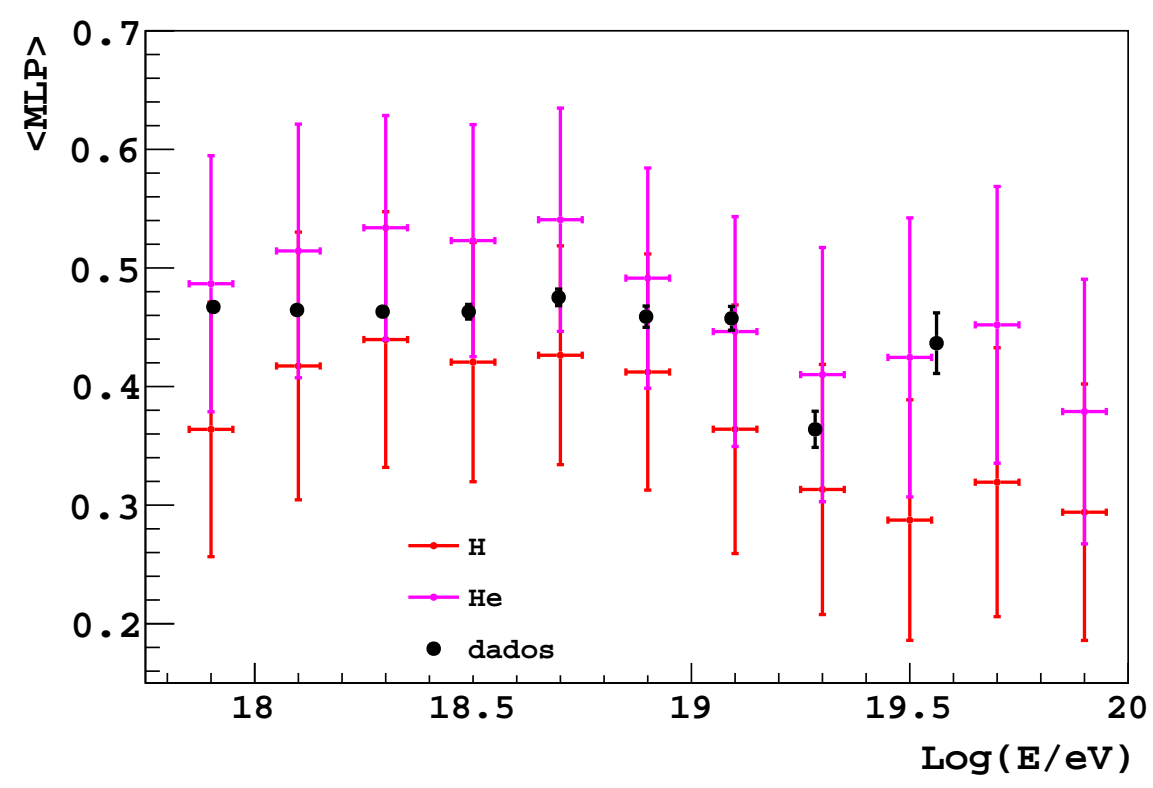

Figura 5.16 - Evolução do parâmetro $M L P$ com a energia para simulações de núcleos de hidrogênio e hélio e para os dados medidos pelo Observatório Pierre Auger. Fonte: Imagem elaborada pelo autor. 


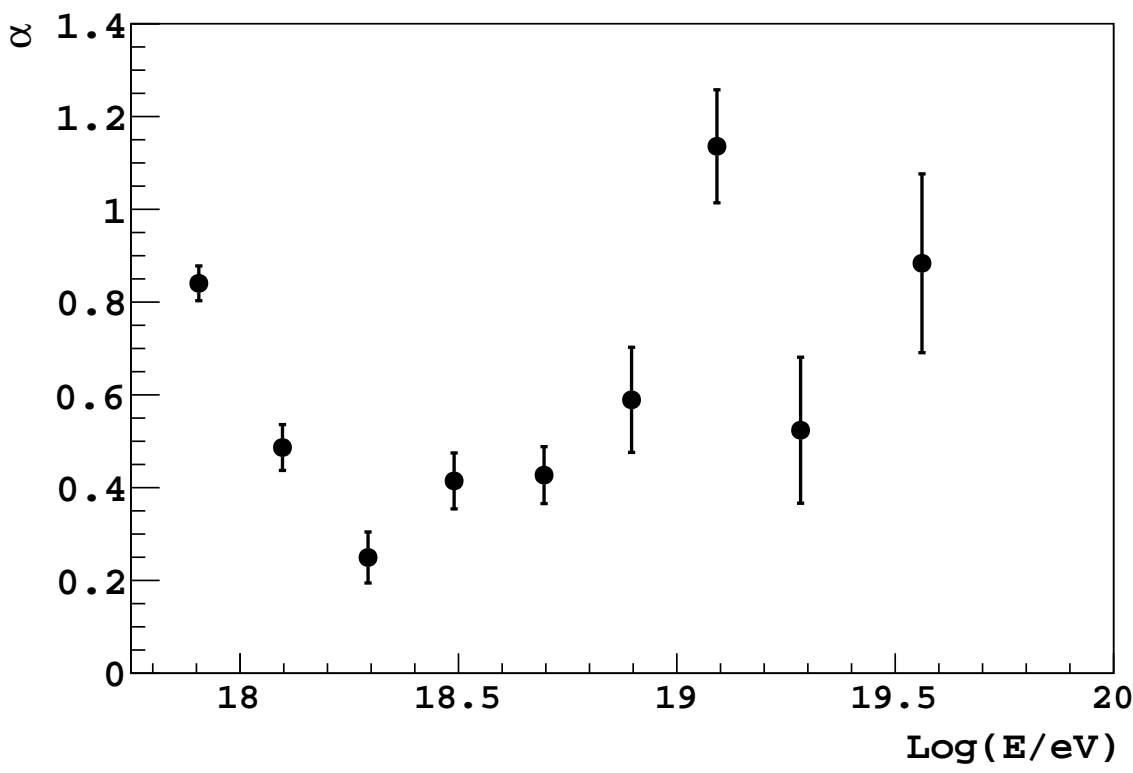

Figura 5.17 - Evolução do parâmetro $\alpha$ com a energia. Fonte: Imagem elaborada pelo autor.

simulações de Monte Carlo.

Na figura 5.18 estão apresentados os valores da resolução energética para a análise desse trabalho (usando redes neurais) e para a reconstrução híbrida padrão (descrita na seção 3.3.3). Notamos que o valor de $\left(E_{r e c}-E_{M C}\right) / E_{M C}$ para a análise com redes neurais oscila em torno de 0 , entretanto na maior parte do espectro de energia é menor que 0.01. No caso da reconstrução híbrida padrão, o valor de $\left(E_{r e c}-E_{M C}\right) / E_{M C}$ é quase constante e da ordem de 0.02. É importante notar que os conjuntos de chuveiros utilizados em ambos os casos foram submetidos aos mesmos critérios de seleção (ver seção 5.5), que por sua vez foram pensados com o objetivo de otimizar a análise por redes neurais e não a análise padrão. Portanto, podemos concluir que a reconstrução da energia primário a partir das redes neurais é mais precisa que a reconstrução padrão para esse conjunto de eventos.

Ainda na figura 5.18 podemos notar que na reconstrução padrão existe uma diferença sistemática entre os diferentes tipos de partículas. Isso acontece por que o cálculo da energia invisível (ver seção 3.3.3) é feito usando informações a priori sobre a composição das partículas. Mais especificamente, esse cálculo supõe que a energia invisível de todos os chuveiros é a média da energia invisível de chuveiro iniciado por prótons e por núcleos de ferro. No caso da análise com redes neurais essa diferença sistemática entre os tipos de partículas não existe. Isso se explica pelo fato da análise por redes neurais também conter informações sobre a composição das partículas e portanto essas diferenças de massa são levadas em conta na reconstrução da energia. 


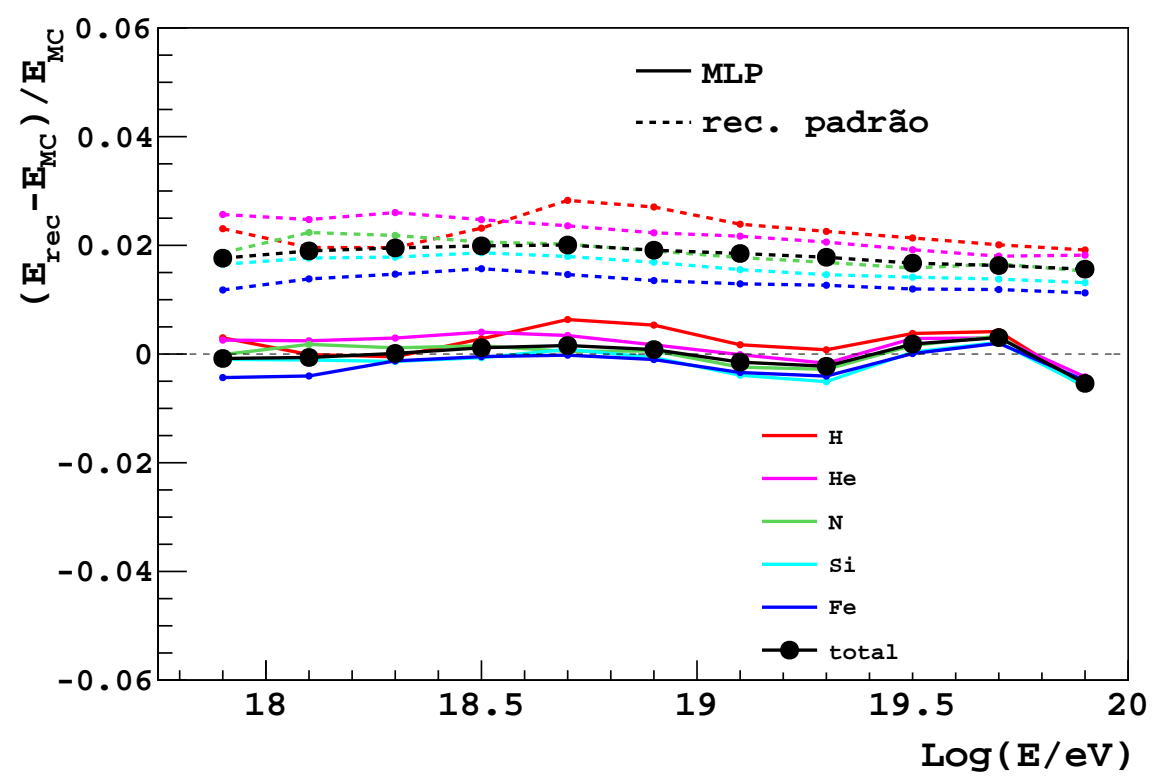

Figura 5.18 - Resolução energética para a análise padrão e a análise desse trabalho obtida através de chuveiros simulados. Fonte: Imagem elaborada pelo autor.

Novamente, como foi feito com o parâmetro $M L P$, avaliaremos a importância de cada parâmetro do chuveiro na reconstrução da energia. A figura 5.19 mostra os valores de $\left(E_{M L P}-\right.$ $\left.E_{M C}\right) / E_{M C}$ em função da energia para todas as combinações em pares dos três parâmetros $X_{\max }, \sigma$ e $\log \left(N_{\max }\right)$ e para o caso em que todos os três parâmetros foram utilizados. Podemos notar claramente que no caso do par $X_{\max }+\sigma$ a qualidade da reconstrução é muito pior e portanto, como já era esperado, podemos concluir que o parâmetro $N_{\text {max }}$ é essencial para a reconstrução da energia. Comparando agora os pares $N_{\max }+X_{\max }$ e $N_{\max }+\sigma$, notamos que a qualidade da reconstrução é levemente melhor no caso do par $N_{\max }+\sigma$, ou seja, o parâmetro $\sigma$ contém mais informação sobre a energia do que $\circ X_{\max }$. Esse fato pode ser entendido, uma vez que o $X_{\max }$ é apenas uma medida do deslocamento do perfil longitudinal, não influenciando fortemente em sua forma. Ao contrário disso, o $\sigma$ é uma medida da largura do perfil e portanto influencia diretamente na forma e na integral do perfil longitudinal, e consequentemente na energia calculada do chuveiro.

A figura 5.20 mostra a diferença relativa na energia dos eventos medidos pelo Observatório Pierre Auger calculada através da análise por redes neurais desenvolvida neste trabalho e da análise padrão. Notamos que existe uma diferença significativa, porém sempre menor que $5 \%$. Apesar do foco desse trabalho ser a reconstrução da massa da partícula primária, podemos concluir que as redes neurais, como utilizadas aqui, podem ser utilizadas para reconstruir a energia de eventos híbridos. Entretanto, os cortes mostrados na seção 5.5 são essenciais para 


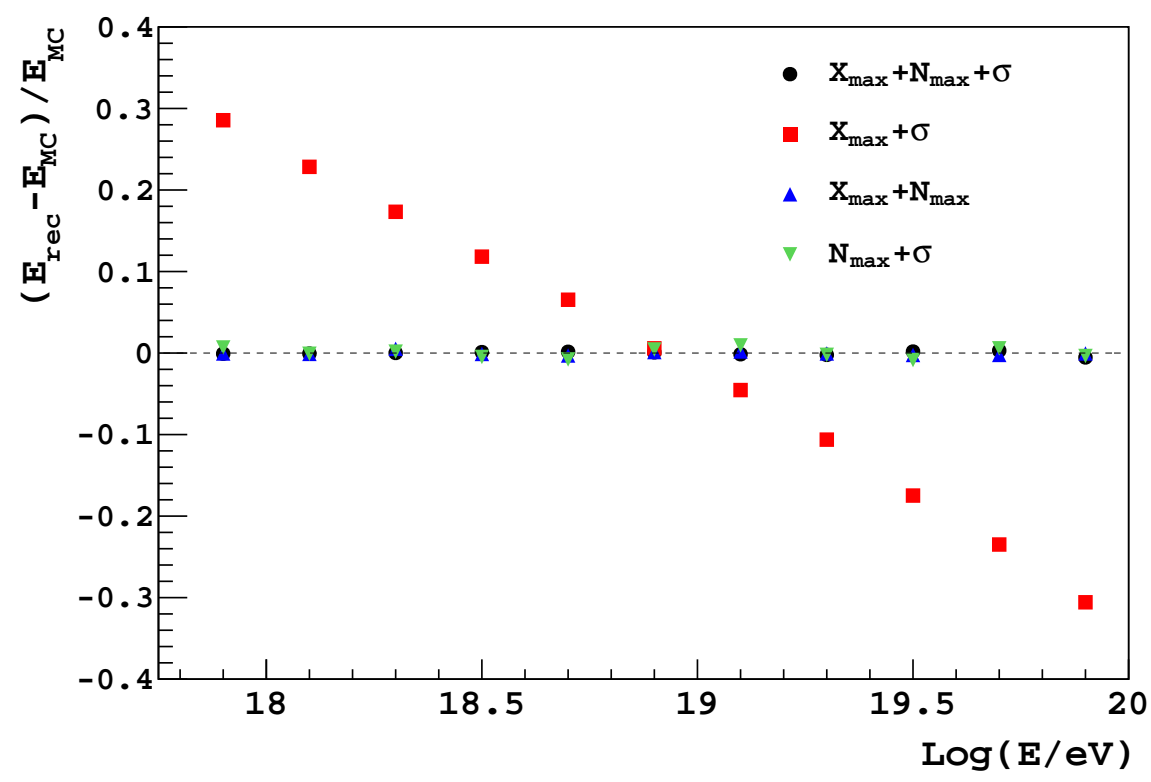

Figura 5.19 - Resolução energética para a análise desse trabalho usando $X_{\max }, \sigma$ e $\log \left(N_{\max }\right)$ e usando pares desses parâmetros. Fonte: Imagem elaborada pelo autor.

a qualidade desta análise e geram como consequência negativa a diminuição do número de eventos disponíveis. Uma análise mais profunda a cerca dos diferentes critérios de seleção dos eventos e dos números de eventos em cada caso seria necessária para avaliar a viabilidade de nossa técnica como método alternativo de reconstrução híbrida de energiar. Entretanto, essa etapa não será apresentada nessa dissertação e será realizada em trabalhos futuros. 


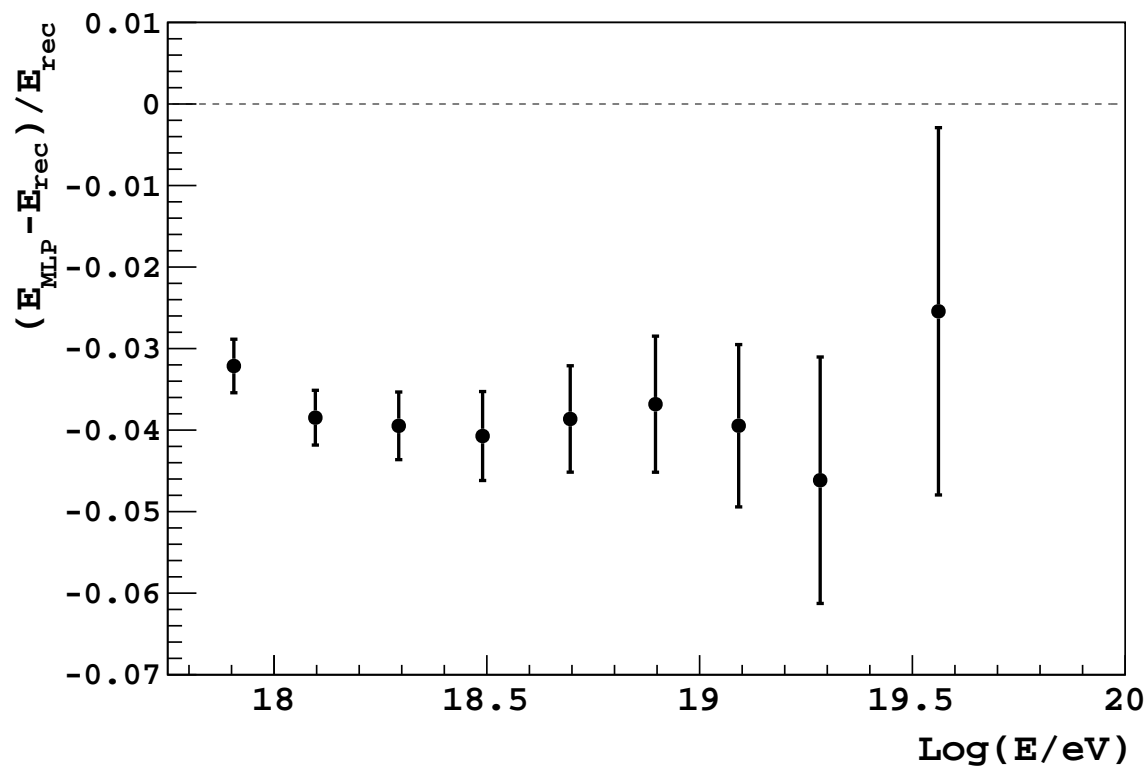

Figura 5.20 - Diferença entre a energia reconstruída pelo método de redes neurais, apresentada nesse trabalho, e a energia reconstruída pelo método padrão para os dados medidos pelo Observatório Pierre Auger. Fonte: Imagem elaborada pelo autor. 


\section{Conclusões}

Nesse trabalho foram apresentados duas análises de composição de raios cósmicos de altas energias usando dados medidos pelo Observatório Pierre Auger. Nos dois casos foram utilizados eventos híbridos medidos pelos detectores de superfície e pelos telescópios de fluorescência. No capítulo 4 apresentamos uma análise das distribuições de $X_{\max }$ através de métodos de deconvolução. As conclusões referentes a essa etapa serão apresentadas na seção 6.1. A segunda análise, apresentada no capítulo 5, aplica redes neurais aos parâmetros extraídos dos perfis longitudinais dos chuveiros com a intenção de inferir a massa e a energia da partícula inicial. Na seção 6.2 serão apresentadas as conclusões referentes a essa segunda análise. Para finalizar, faremos as conclusões gerais do trabalho na seção 6.3

\subsection{Taxa de elongação: métodos de deconvolução}

Os métodos de deconvolução foram desenvolvidos com o objetivo de extrair os efeitos dos detectores das distribuições medidas por experimentos. Neste trabalho, aplicamos dois métodos de deconvolução (SVD e iterativo bayesiano) às distribuições de $X_{\max }$ medidas pelo Observatório Pierre Auger. A número de eventos selecionados para essa análise foi da ordem de 2 vezes maior que o número de eventos utilizado na análise padrão, como pode ser visto na figura 4.6. O resultado dessa diferença no número de eventos foi a dimuição significativa da incerteza estatística, como mostrado nas figuras 4.7 e 4.8

Para verificar a eficiência dos métodos implementados e avaliar os possíveis viéses adicionados pela análise, foram realizados testes de consistência (seção 4.3). Nesses testes, aplicamos os métodos de deconvolução à distribuições de $X_{\max }$ obtidas por simulações, em que tanto a distribuição real, como a distribuição enviesada pelos efeitos dos detectores, eram conhecidas. Comparando os valores médios e a variância dessas distribuições notamos uma diferença máxima de $4 \mathrm{~g} / \mathrm{cm}^{2}$ para ambos os casos (figura 4.5). Como a incerteza sistemática da reconstrução dos eventos é significantemente maior que essas diferenças (4.4.3), concluímos que os resultados do teste de consistência são suficientes para validar os resultados obtidos pelos 
métodos de deconvolução.

As incertezas estatísticas e sistemáticas foram avalidas nas seções 4.4 .2 e 4.4.3. Na seção 4.5 apresentamos os resultados gerais da análise de deconvolução. Primeiramente, pelas figuras 4.11 e 4.12, foi possível observar a concordância entre os resultados para os dois diferentes métodos. Isso pode ser concluído pelo fato da diferença entre os valores de $\left\langle X_{\max }\right\rangle \mathrm{e}$ $R M S_{X_{\max }}$, calculados a partir das distribuições deconvoluídas pelos dois métodos, é menor ou da ordem das incertezas estatísticas envolvidas. Uma vez que foram escolhidos duas técnicas que se baseiam em princípios matemáticos completamente diferentes, a concordância entre os resultados é uma forte evidência que não houve falhas nas implementações dos algoritmos e que os resultados são válidos.

As figuras 4.18 e 4.16 resumem os resultados obtidos referentes a composição dos raios cósmicos. Pelo cálculo de $\langle\ln (A)\rangle$ apresentado na figura 4.16 podemos concluir que, de acordo com os modelos de interação hadrônica, a massa média das partículas medidas apresenta dois comportamentos distintos com relação a energia. Assumindo como corretas as predições das simulações, concluímos que em valores baixos de energia a composição é praticamente constante e leve em média. Em aproximadamente $10^{18.4} \mathrm{eV}$ vemos que existe uma transição e a partir desse valor a massa média tende a aumentar.

\subsection{Análise multiparamétrica: redes neurais}

As redes neurais do tipo Multilayer Perceptrons são comumente utilizadas como métodos de inferência estatística multiparamétricos. Neste trabalho, essa ferramenta foi aplicada aos parâmetros extraídos dos perfil longitudinal dos chuveiros atmosféricos.

Os resultados apresentados na seção 5.7.1 mostram que foi possível obter um parâmetro mais sensível a composição do que os parâmetros extraídos diretamente do perfil longitudinal. Isso pode ser observado na figura 5.11 que mostra o fator de mérito para os parâmetros $M L P$, $X_{\max }, \sigma$ e $\log \left(N_{\max }\right)$. A evolução de $M L P$ com a energia (figuras 5.16 e 5.17 ) mostra uma transição em aproximadamente $10^{18.3} \mathrm{eV}$ em que a massa média tende a se tornar mais pesada, uma vez que assumimos como corretas as predições das simulações.

Além da composição, a análise por redes neurais foi utilizada para a reconstrução da energia das partículas primárias. Na seção 5.7.2, foi mostrado que a resolução energética para essa análise é melhor que a resolução para a reconstrução padrão, para o conjunto de simulações selecionadas nesse trabalho. Entretanto, para validar nossa análise como método 
alternativo de reconstrução da energia, ainda são necessários estudos mais aprofundados sobre a seleção dos eventos e como esta interfere na resolução energética obtida. Essa etapa será realizada em trabalhos futuros.

Uma característica muito importante da análise por redes neurais do tipo Multilayer Perceptrons é a sua forte dependência com relação às simulações. Nesse trabalho, os chuveiros utilizados durante a etapa de treinamento foram gerados através do modelo de interação hadrônica QGSJetll-04 (99, 100). Como o custo computacional da geração desses chuveiros simulados é muito alto, a repetição da análise com outros modelos de interação hadrônica não foi viável no intervalo de tempo disponível para realização dessa dissertação. Essa tarefa será realizada em trabalhos futuros.

\subsection{Conclusões gerais}

As duas análises apresentadas nessa dissertação foram baseadas nos dados medidos pelos telescópios de fluorescência do Observatório Pierre Auger. Nos dois casos os resultados mostraram uma transição quanto a composição média das partículas em aproximadamente $10^{18.3}$ - $10^{18.4} \mathrm{eV}$. Para energias abaixo desse valor a massa média das partículas é mais baixa e acima existe uma tendência à partículas mais pesadas. Essas conclusões se baseiam na consideração que as predições das simulações estão corretas. A figura 6.1 mostra um resumo dos resultados de composição obtidos nessa dissertação.

Essa região de transição coincide com a região do "tornozelo" do espectro de energia. Isso pode indicar que os fenômenos astrofísicos responsáveis por gerarem essa estrutura podem ser os mesmos responsáveis pela mudança de composição. 

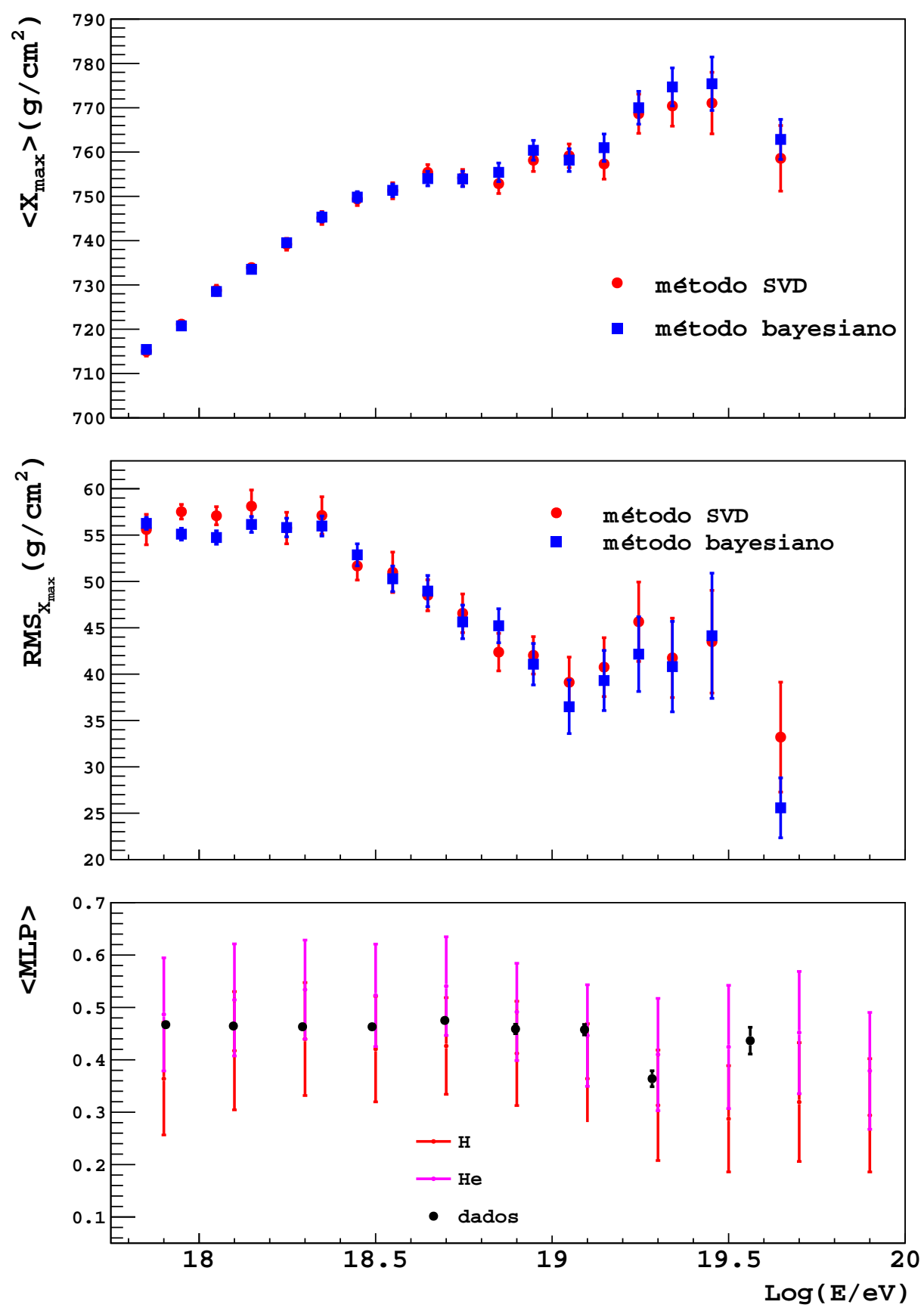

Figura 6.1 - Resumo dos resultados apresentados nessa dissertação. O primeiro gráfico mostra o $\left\langle X_{\max }\right\rangle$ em função da energia para os métodos de deconvolução. O segundo mostra o $R M S_{X_{\max }}$ em função da energia para os métodos de deconvolução. O último mostra o parâmetro $\langle M L P\rangle$ obtido através da análise por redes neurais em função da energia. Fonte: Imagem elaborada pelo autor. 


\section{REFERÊNCIAS}

1 KAMPERT, K.-H.; WATSON, A. A. Extensive air showers and ultra high-energy cosmic rays: a historical review. The European Physical Journal H, v. 37, n. 3, p. 359-412, July 2012.

2 ABRAHAM, J. et al. Properties and performance of the prototype instrument for the pierre auger observatory. Nuclear Instruments and Methods in Physics Research Section A: accelerators, spectrometers, detectors and associated equipment, v. 523, n. 1-2, p. 50-95, May 2004.

3 AHN, E. J. et al. Measurement of the depth of shower maximum of cosmic rays above $10^{18} \mathrm{eV}$. [s.I]: Pierre Auger Observatory, 2009. Relatório técnico, GAP-2009-078

4 HöCKER, A.; KARTVELISHVILI, V. SVD approach to data unfolding. Nuclear Instruments and Methods in Physics Research Section A: accelerators, spectrometers, detectors and associated equipment, v. 372, n. 3, p. 469-481, Apr. 1996.

5 D'AGOSTINI, G. A multidimensional unfolding method based on Bayes' theorem. Nuclear Instruments and Methods in Physics Research Section A: accelerators, spectrometers, detectors and associated equipment, v. 362, n. 2-3, p. 487-498, Aug. 1995.

6 BISHOP, C. M. Neural networks and their applications. Review of Scientific Instruments, Woodbury, v. 65, n. 6, p. 1803, 1994.

7 BHATTACHARJEE, P.; HILL, C. T.; SCHRAMM, D. N. Grand unified theories, topological defects, and ultrahigh-energy cosmic rays. Physical Review Letters, v. 69, n. 4, p. 567-570, 1992.

8 DREES, M. The top-down interpretation of ultra-high energy cosmic rays. Journal of the Physical Society of Japan, v. 77, p. 16-18, 2008.

9 TORRES, D. F.; ANCHORDOQUI, L. A. Astrophysical origins of ultrahigh energy cosmic rays. Reports on Progress in Physics, v. 67, n. 9, p. 1663-1730, Sept. 2004.

10 NELLEN, L.; SáNCHEZ, F. A.; VALDéS-GALICIA, J. F. Constraints on top-down models for the origin of UHECRs from the Pierre Auger Observatory data super-heavy dark matter scenarios. In: INTERNATIONAL COSMIC RAYS CONFERENCE, 30., 2007. Mérida, Proceedings... Mérida, Yucatan, México: [s.n], 2007.

11 ABRAHAM, J. et al. Upper limit on the cosmic-ray photon fraction at EeV energies from the Pierre Auger Observatory. Astroparticle Physics, v. 31, n. 6, p. 399-406, July 2009. 
12 FERMI, E. On the origin of the cosmic radiation. Physical Review, v. 105, n. 1943, p. 4-9, 1949.

13 HILLAS, A. M. The Origin of Ultra-High-Energy Cosmic Rays. Annual Review of Astronomy and Astrophysics, v. 22, n. 1, p. 425-444, Sept. 1984.

14 BLASI, P.; EPSTEIN, R. I.; OLINTO, A. V. Ultra-high-energy cosmic rays from young neutron star winds. The Astrophysical Journal, v. 533, p. L123-L126, 2000.

15 BLüMER, J.; ENGEL, R.; HöRANDEL, J. R. Cosmic rays from the knee to the highest energies. Progress in Particle and Nuclear Physics, v. 63, n. 2, p. 293-338, Oct. 2009.

16 HöRANDEL, J. R. Models of the knee in the energy spectrum of cosmic rays. Astroparticle Physics, v. 21, n. 3, p. 241-265, June 2004.

17 APEL, W. D. et al. Kneelike structure in the spectrum of the heavy component of cosmic rays observed with kascade-grande. Physical Review Letters, v. 107, p. 171104, Oct 2011.

18 ALOISIO, R.; BEREZINSKY, V.; GAZIZOV, A. Transition from galactic to extragalactic cosmic rays. Astroparticle Physics, v. 39-40, p. 129-143, Dec. 2012.

19 GREISEN, K. End to the cosmic-ray spectrum? Physical Review Letters, v. 16, n. 17, p. 748-750, 1966.

20 ZATSEPIN, G. T.; KUZ'MIN, V. A. Upper limit of the spectrum of cosmic rays. Soviet Journal of Experimental and Theoretical Physics Letters, v. 4, p. 78-80, 1966.

21 ABRAHAM, J. et al. Observation of the suppression of the flux of cosmic rays above $4 \times 10^{19} \mathrm{eV}$. Physical Review Letters, v. 101, n. 6, p. 061101, Aug. 2008.

22 ABBASI, R. et al. First observation of the Greisen-Zatsepin-Kuzmin suppression. Physical Review Letters, v. 100, n. 10, p. 101101, Mar. 2008.

23 TAKEDA, M. et al. Energy determination in the Akeno giant air shower array experiment. Astroparticle Physics, v. 19, n. 4, p. 447-462, July 2003.

24 GAISSER, T. K.; STANEV, T.; TILAV, S. Cosmic ray energy spectrum from measurements of air showers. Front. Phys. China, v. 8, p. 748-758, Mar. 2013.

25 ANCHORDOQUI, L. et al. High energy physics in the atmosphere: phenomenology of cosmic ray air showers. Annals of Physics, New York, v. 314, n. 1, p. 145-207, Nov. 2004.

26 ABREU, P. et al. Measurement of the proton-air cross section at $\sqrt{s}=57 \mathrm{TeV}$ with the Pierre Auger Observatory. Physical Review Letters, v. 109, n. 6, p. 062002, Aug. 2012. 
27 KAMPERT, K.H.; UNGER, M. Measurements of the cosmic ray composition with air shower experiments. Astroparticle Physics, v. 35, n. 10, p. 660-678, May 2012.

28 HECK, D.; Corsika: a Monte Carlo code to simulate extensive air showers. Karlsruhe: FZKA, 1998.

29 SCIUTTO, S. J. AIRES: a system for air shower simulations. 1999. Disponível em: <http://arxiv.org/pdf/astro-ph/9911331v1.pdf>. Acesso em: jan 13, 2014

30 PIEROG, T. et al. First results of fast one-dimensional hybrid simulation of EAS using conex. Nuclear Physics B-Proceedings Supplements, v. 151, n. 1, p. 159-162, Jan. 2006.

31 BERGMANN, $T$. et al. One-dimensional hybrid approach to extensive air shower simulation. Astroparticle Physics, v. 26, n. 6, p. 420-432, Jan. 2007.

32 BEREZHNEV, S. et al. The Tunka-133 EAS cherenkov light array: status of 2011. Nuclear Instruments and Methods in Physics Research Section A: accelerators, spectrometers, detectors and associated equipment, v. 692, p. 98-105, Nov. 2012.

33 KNURENKO, S. P.; SABOUROV, A. Study of cosmic rays at the Yakutsk EAS array: energy spectrum and mass composition. Nuclear Physics B-Proceedings Supplements, v. 212-213, p. 241-251, Mar. 2011.

34 FOWLER, J. et al. A measurement of the cosmic ray spectrum and composition at the knee. Astroparticle Physics, v. 15, n. 1, p. 49-64, Mar. 2001.

35 OVAULT, C. E. C. et al. Measurement of the cosmic-ray energy spectrum and composition from $10^{17}$ to $10^{18.3} \mathrm{eV}$ using a hybrid technique. The Astrophysical Journal, v. 20, p. 686-699, 2001.

36 ABBASI, R. U. et al. Indications of proton-dominated cosmic-ray composition above 1.6 EeV. Physical Review Letters, v. 104, n. 16, p. 161101, Apr. 2010.

37 JUI, C. C. H. Cosmic ray in the northern hemisphere: results from the Telescope Array experiment. In: MEETING OF AMERICAN PHYSICAL SOCIETY, DIVISION OF PARTICLE AND FIELDS, 2011. Santa Cruz. Proceedings... Santa Cruz: Santa Cruz Institute for Particle Physics, 2011.

38 FACAL SAN LUIS, P. The distribution of shower maxima of UHECR air showers. In: INTERNATIONAL COSMIC RAYS CONFERENCE, 32.,2011. Beijing. Proceedings... Beijing: CAS, 2011.

39 ANTONI, T. et al. The cosmic-ray experiment KASCADE. Nuclear Instruments and Methods in Physics Research Section A: accelerators, spectrometers, detectors and associated equipment, v. 513, n. 3, p. 490-510, Nov. 2003. 
40 APEL, W. et al. The KASCADE-Grande experiment. Nuclear Instruments and Methods in Physics Research Section A: accelerators, spectrometers, detectors and associated equipment, v. 620, n. 2-3, p. 202-216, Aug. 2010.

41 FUHRMANN, D. et al. KASCADE-Grande measurements of energy spectra for elemental groups of cosmic rays. In: INTERNATIONAL COSMIC RAYS CONFERENCE, 32., 2011. Beijing. Proceedings... Beijing: CAS, 2011.

42 APEL, W. D. et al. Ankle-like feature in the energy spectrum of light elements of cosmic rays observed with KASCADE-Grande. Physical Review D, v. 87, n. 8, p. 081101, Apr. 2013.

$43 \mathrm{SCHOO}, \mathrm{S}$. et al. The energy-spectrum of light primaries in the range from $10^{16.6}$ to $10^{18.2} \mathrm{eV}$. In: INTERNATIONAL COSMIC RAYS CONFERENCE, 33., 2013. Rio de Janeiro. Proceedings... Rio de Janeiro:[s.n] , 2013

44 PENTCHEV, L.; DOLL, P. Method for a reconstruction of the muon longitudinal and lateral distributions in hadronic extensive air showers. Journal of Physics G: nuclear and particle physics, v. 27, p. 1459-1473, 2001.

45 ANDRINGA, S. et al. The muonic longitudinal shower profiles at production. Astroparticle Physics, v. 35, n. 12, p. 821-827, July 2012.

46 GARCÍA-GáMEZ, D. Measurement of atmospheric production depths of muons with the Pierre Auger Observatory MPD reconstruction. In: INTERNATIONAL COSMIC RAYS CONFERENCE, 32., 2011. Beijing. Proceedings... Beijing: CAS, 2011.

47 DOVA, M. et al. Time asymmetries in extensive air showers: a novel method to identify UHECR species. Astroparticle Physics, v. 31, n. 4, p. 312-319, May 2009.

48 GARCíA-GáMEZ, D.; ARQUEROS, F.; MONASOR, M. Time asymmetries in extensive air showers : a novel method to identify UHECR species. In: INTERNATIONAL COSMIC RAYS CONFERENCE, 31., 2009. Lódz. Proceedings... Lódz: University of Lódz , 2011.

49 ALLEKOTTE, I. et al. Site survey for the Pierre Auger observatory. Journal of Physics G: nuclear and particle physics, v. 28, n. 6, p. 1499-1509, June 2002.

50 LINSLEY, J. Evidence for a primary cosmic-ray particle with energy $10^{20} \mathrm{eV}$. Physical Review Letters, v. 10, n. 4, p. 146, 1962.

51 HASHIMOTO, K. et al. Akeno giant air shower array ( AGASA ) covering $100 \mathrm{~km}^{2}$ area. Nuclear Instruments and Methods in Physics Research Section A: accelerators, spectrometers, detectors and associated equipment, v. 311, n. 1-2, p. 338-349, 1992. 
52 LAWRENCE, M. A.; REID, R. J.; WATSON, A. A. The cosmic ray energy spectrum above $4 \times 10^{17} \mathrm{eV}$ as measured by Haverah Park array. Journal of Physics G: nuclear and particle physics, v. 17, n. 5, p. 733, 1991.

53 BALTRUSAITIS, R. M. et al. The utah fly's eyes detector. Nuclear Instruments and Methods in Physics Research Section A: accelerators, spectrometers, detectors and associated equipment, v. 240, n. 2, p. 410-428, 1985.

54 BOYER, J. H. et al. Fadc-based daq for hires fly's eye. Nuclear Instruments and Methods in Physics Research Section A: accelerators, spectrometers, detectors and associated equipment, v. 482, n. 1-2, p. 457-474, 2002.

55 ABU-ZAYYAD, $T$. et al. The cosmic ray energy spectrum observed with the surface detector of the Telecope Array experiment. The Astrophysical Journal, v. 768, n. 1, p. L1, May 2013.

56 ABRAHAM, J. et al. The fluorescence detector of the pierre auger observatory. Nuclear Instruments and Methods in Physics Research Section A: accelerators, spectrometers, detectors and associated equipment, v. 620, n. 2-3, p. 227-251, Aug. 2010.

57 AVE, M. et al. The accuracy of signal measurement with the water cherenkov detectors of the pierre auger observatory. Nuclear Instruments and Methods in Physics Research Section A: accelerators, spectrometers, detectors and associated equipment, v. 578 , n. 1, p. 180-184, July 2007.

58 ALLEKOTTE, I. et al. The surface detector system of the Pierre Auger Observatory. Nuclear Instruments and Methods in Physics Research Section A: accelerators, spectrometers, detectors and associated equipment, v. 586, n. 3, p. 409-420, Mar. 2008.

59 ABRAHAM, J. et al. Trigger and aperture of the surface detector array of the pierre auger observatory. Nuclear Instruments and Methods in Physics Research Section A: accelerators, spectrometers, detectors and associated equipment, v. 613, n. 1, p. 29-39, Jan. 2010.

60 ALLARD, D. et al. Aperture calculation of the pierre auger observatory surface detector. In: INTERNATIONAL COSMIC RAYS CONFERENCE, 29., 2005. Pune, Índia. Proceedings... Pune, Índia: [s.n], 2005.

61 MEURER, C.; SCHARF, N. HEAT - a low energy enhancement of the Pierre Auger Observatory. In: EUROPEAN COSMIC RAYS SYMPOSIUM, 22., 2010. Turku, Finland. Proceedings... Turku, Finland: [s.n], 2010.

62 HORVAT, M.; VEBERI, D. On optimal barycenter estimation. [s.I]: Pierre Auger Observatory, 2007. Relatório técnico, GAP-2007-035. 
63 ROTH, M.; POLYTECHNIC, N. G. Offline reference manual. [s.I]: Pierre Auger Observatory, 2005. Relatório técnico, GAP-2005-035.

64 BONIFAZI, C. The angular resolution of the Pierre Auger Observatory. Nuclear Physics B- Proceedings Supplements, v. 190, p. 20-25, May 2009.

65 KUEMPEL, D.; KAMPERT, K.; RISSE, M. Geometry reconstruction of fluorescence detectors revisited. Astroparticle Physics, v. 30, n. 4, p. 167-174, Nov. 2008.

66 MOSTAFá, M. Hybrid activities of the Pierre Auger Observatory. Nuclear Physics B Proceedings Supplements, v. 165, p. 50-58, Mar. 2007.

$67 \mathrm{ROTH}, \mathrm{M}$. The lateral distribution function of shower signals in the surface detector of the pierre auger observatory. In: INTERNATIONAL COSMIC RAYS CONFERENCE, 28., 2003. Tsukuba, Japan. Proceedings... Tsukuba, Japan: [s.n], 2011.

68 NEWTON, D.; KNAPP, J.; WATSON, A. The optimum distance at which to determine the size of a giant air shower. Astroparticle Physics, v. 26, n. 6, p. 414-419, Jan. 2007.

69 KAMATA, K.; NISHIMURA, J. The lateral and the angular structure functions of electron showers. Progress of Theoretical Physics Supplement, v. 6, p. 93-155, 1958.

70 PUPPI, G.; BRIDGE, H.; GREISEN, K. Progress in cosmic ray physics. Vol. 3. New York: North-Holland, 1956.

71 PEIXOTO, C. J. T. Reconstrução de chuveiros atmosféricos extensos detectados pelo Observatório Pierre Auger utilizando métodos robustos. 2008. 118 p. Tese (Doutorado em Física) - Instituto de Física Gleb Wataghin, Universidade Estadual de Campinas, Campinas, 2008.

72 BARNHILL, D. et al. Measurement of the lateral distribution function of uhecr air showers with the pierre auger observatory. In: INTERNATIONAL COSMIC RAYS CONFERENCE, 29., 2005. Pune, Índia. Proceedings... Pune, Índia: [s.n], 2005.

73 DI GIULIO, C. Energy calibration of data recorded with the surface detectors of the Pierre Auger Observatory. In: INTERNATIONAL COSMIC RAYS CONFERENCE, 31., 2009 Lódz. Proceedings... Lódz: University of Lódz, 2009.

74 UNGER, M. et al. Reconstruction of longitudinal profiles of ultra-high energy cosmic ray showers from fluorescence and Cherenkov light measurements. Nuclear Instruments and Methods in Physics Research Section A: accelerators, spectrometers, detectors and associated equipment, v. 588, n. 3, p. 433-441, Apr. 2008.

75 GAISSER, T. K.; HILLAS, A. M. Reliability of the method of constant intensity cuts for reconstructing the average development of vertical showers. In: INTERNATIONAL COSMIC 
RAYS CONFERENCE, 15., 1977. Plovdiv, Bulgaria. Proceedings... Plovdiv, Bulgaria: [s.n], 1977.

76 BARBOSA, H. M. J. et al. Determination of the calorimetric energy in extensive air showers. Astroparticle Physics, v. 22, n. 2, p. 159-166, Nov. 2004.

77 ROTH. Measurement of the UHECR energy spectrum using data from the surface detector of the Pierre Auger Observatory. In: INTERNATIONAL COSMICS RAYS CONFERENCE, 30., 2007. Mérida. Proceedings... Mérida, Yucatan, México: [s.n], 2007.

78 HANSEN, P. Discrete inverse problems: insight and algorithms. Society for Industrial and Applied Mathematics (Philadelphia, PA-USA: SIAM), 2010.

79 BLOBEL, V. Unfolding methods in high-energy physics experiments. In: CERN SUMMER SCHOOL FOR COMPUTING, 1984 Aiguablava, Spain. Proceedings... Aiguablava, Spain: [s.n], 1984.

80 ANYKEYEV, V.; SPIRIDONOV, A.; ZHIGUNOV, V. Comparative investigation of unfolding methods. Nuclear Instruments and Methods in Physics Research Section A: accelerators, spectrometers, detectors and associated equipment, v. 303, n. 2, p. 350-369, June 1991.

81 COWAN, G. Statistical data analysis. Clarendon Press, 1998.

82 JONKER, M. et al. Experimental study of differential cross sections in neutral current neutrino and antineutrino interactions. Physics Letters B, Amsterdam, v. 102, n. 1, p. 67-72, June 1981.

83 JONKER, M. et al. Experimental study of $\mathrm{x}$-distributions in semileptonic neutral-current neutrino reactions. Physics Letters B, Amsterdam, v. 128, n. 1-2, p. 117-123, Aug. 1983.

$84 \mathrm{AAD}, \mathrm{G}$. et al. Charged-particle multiplicities in pp interactions measured with the ATLAS detector at the LHC. New Journal of Physics, v. 13, n. 5, p. 053033, May 2011.

$85 \mathrm{AIOLA}, \mathrm{S}$. Measurement of jet $\mathrm{p} \mathrm{T}$ spectra in $\mathrm{Pb}-\mathrm{Pb}$ collisions at $=2.76 \mathrm{TeV}$ with the ALICE detector at the LHC. Journal of Physics: conference series, v. 446, p. 012005, Sept. 2013.

86 GANGULY, S.; GUCHAIT, M. Jet croos-section measurements in CMS. International Journal of Modern Physics A, Singapore, v. 28, n. 20, p. 1330030, Aug. 2013.

87 ANTONI, T. et al. KASCADE measurements of energy spectra for elemental groups of cosmic rays: results and open problems. Astroparticle Physics, v. 24, n. 1-2, p. 1-25, Sept. 2005. 
88 APEL, W. et al. Energy spectra of elemental groups of cosmic rays: update on the KASCADE unfolding analysis. Astroparticle Physics, v. 31, n. 2, p. 86-91, Mar. 2009.

89 APEL, W. et al. KASCADE-Grande measurements of energy spectra for elemental groups of cosmic rays. Astroparticle Physics, v. 47, n. 1, p. 54-66, Jun. 2013.

90 ABRAHAM, J. et al. Measurement of the depth of maximum of extensive air showers above $10^{18} \mathrm{eV}$. Physical Review Letters, v. 104, n. 9, Mar. 2010.

91 LAWSON, C. L.; HANSON, R. J. Solving least squares problems New edition. ed. Society for Industrial Mathematics, 1987. (classics in applied mathematics).

92 HECK, D. et al. CORSIKA: a Monte Carlo Code to simulate extensive air showers. Relatório técnico, Forschungszentrum Karlsruhe, Karlsruhe, 1998. Relatio técnico - Report FZKA 6019.

93 FLETCHER, R. et al. SIBYLL: an event generator for simulation of high energy cosmic ray cascades. Physical Review D, v. 50, n. 9, p. 5710-5731, Nov. 1994.

94 PERRONE, L.; PETRERA, S.; SALAMIDA, F. Gaisser-Hillas profiles and FD simulations. [s.I]: Pierre Auger Observatory, 2005. Relatório técnico, GAP-2005-087.

95 ARGIRO, S. et al. The offline software framework of the Pierre Auger Observatory. Nuclear Instruments and Methods in Physics Research Section A: accelerators, spectrometers, detectors and associated equipment, v. 580, n. 3, p. 1485-1496, Oct. 2007.

96 AMSLER, C. et al. Mathematical tools or statistics, Monte Carlo, group theory. Physics Letters B, Amsterdam, v. 667, n. 1-5, p. 316-339, Sept. 2008.

97 PEIXOTO, C. J. T.; DE SOUZA, V.; BELLIDO, J. A. Comparison of the moments of the distribution predicted by different cosmic ray shower simulation models. Astroparticle Physics, v. 47, n. 0 , p. $18-30,2013$.

98 AHN, E. J. Inferences about the mass composition of cosmic rays from data on the depth of maximum at the Auger Observatory. In: INTERNATIONAL COSMIC RAYS CONFERENCE, 33., 2013. Rio de Janeiro. Proceedings... Rio de Janeiro:[s.n] , 2013.

99 OSTAPCHENKO, S. QGSJET-II: towards reliable description of very high energy hadronic interactions. Nuclear Physics B-Proceedings Supplements, v. 151, n. 1, p. 143-146, Jan. 2006.

100 STAPCHENKO, S. O. Air shower development: impact of LHC data 2 QGSJET-II model : physics update Comparison with LHC data. In: INTERNATIONAL COSMIC RAYS CONFERENCE, 32., 2011. Beijing. Proceedings... Beijing: CAS, 2011. 
101 PIEROG, T.; WERNER, K. Muon production in extended air shower simulations. Physical Review Letters, v. 101, n. 17, p. 171101, Oct. 2008.

102 BHAT, P. C. Multivariate analysis methods in particle physics. Annual Review of Nuclear and Particle Science, v. 61, n. 1, p. 281-309, Nov. 2011.

103 MEDINA-TANCO, G.; SCIUTTO, S. A multivariate study of mass composition for simulated showers at teh Auger South Observatory. In: INTERNATIONAL COSMIC RAYS CONFERENCE, 27., 2001. Hamburg. Proceedings... Hamburg: [s.n], 2001.

104 AMBROSIO, M. et al. Comparison between methods for the determination of the primary cosmic ray mass composition from the longitudinal profile of atmospheric cascades. Astroparticle Physics, v. 24, n. 4-5, p. 355-371, Dec. 2005.

105 CATALANI, F. et al. Statistical methods applied to composition studies of ultrahigh energy cosmic rays. Astroparticle Physics, v. 28, n. 3, p. 357-365, Nov. 2007.

106 CASTELLINA, A. Comparison of data from the Pierre Auger Observatory with predictions from air shower simulations: testing models of hadronic interactions. In: INTERNATIONAL COSMIC RAYS CONFERENCE, 29., 2009. Lódz. Proceedings... Lódz: University of Lódz , 2009.

107 CAZON, L.; CONCEICãO, R.; ESPIRITO-SANTO, C. Consistency between $X_{\max }$ and $X_{\max }^{\mu}$ as a way to test hadronic models. [s.I]: Pierre Auger Observatory, 2012. Relatório técnico, GAP-2012-068

108 VALIñO, I. A measurement of the muon number in showers using inclined events recorded at the Pierre Auger Observatory. In: INTERNATIONAL COSMIC RAYS CONFERENCE, 33., 2013. Rio de Janeiro. Proceedings... Rio de Janeiro:[s.n] , 2013.

109 CAZON, L.; OLIVEIRA, M.; PIMENTA, M. Number of muons: average, fluctuations and their systematics at $60^{\circ}$. [s.I]: Pierre Auger Observatory, 2013. Relatório técnico, GAP2013-053.

$110 \mathrm{ABBASI}, \mathrm{R}$. et al. Cosmic ray composition and energy spectrum from 1-30PeV using the 40-string configuration of IceTop and IceCube. Astroparticle Physics, v. 42, p. 15-32, Feb. 2013.

111 ANDEEN, K. G. First Measurements of Cosmic Ray Composition from 1-50 PeV using new techniques on coincident data from the IceCube neutrino observatory by. 2011. $189 \mathrm{p}$. Tese (Doutorado em Física) - University of Wisconsin - Madison, 2011.

112 ADRIANI, O. et al. Cosmic-ray positron energy spectrum measured by PAMELA. Physical Review Letters, v. 111, n. 8, p. 081102, Aug. 2013. 
113 ROSSETTO, L. PAMELA measurements of high energy cosmic ray positrons. 2012. p. 160. Tese (Doutorado em Física) - Royal Institute of Technology, 2012.

$114 \mathrm{CAO}, \mathrm{Z}$. A measurement of the longitudinal development profile of cosmic ray air showers between $10^{17} \mathrm{eV}$ and $10^{18} \mathrm{eV}$. In: INTERNATIONAL COSMIC RAYS CONFERENCE, 27., 2001. Hamburg. Proceedings... Hamburg: [s.n], 2001.

115 BROYDEN, C. G. The convergence of a class of double-rank minimization algorithms. IMA Journal of Applied Mathematics, v. 6, p. 76-90, 1970.

116 GOLDFARB, D. A family of variable-metric methods derived by variational means. Mathematics of Computation, v. 24, n. 109, p. 23-26, 1970.

117 FLETCHER, R. A new approach to variable metric algorithms. The Computer Journal, v. 13, n. 3 , p. 317-322, 1970.

118 SHANNO, D. F. Conditioning of quasi-Newton methods for function minimization. Mathematics of Computation, v. 24, n. 111, p. 647-656, 1970.

119 HOECKER, A. et al. TMVA - Toolkit for Multivariate Data Analysis, Mar. 2007. Disponível em:<http://arxiv.org/pdf/physics/0703039v5.pdf>. Acesso em: 13 jan, 2014

120 BRUN, R.; RADEMAKERS, F. ROOT - An object oriented data analysis framework. Nuclear Instruments and Methods in Physics Research Section A: accelerators, spectrometers, detectors and associated equipment, v. 389, n. 1-2, p. 81-86, 1997. 